Additional services for Journal of Fluid Mechanics:

Email alerts: $\underline{\text { Click here }}$

Subscriptions: $\underline{\text { Click here }}$

Commercial reprints: $\underline{\text { Click here }}$

Terms of use : $\underline{\text { Click here }}$

\title{
Vortex/inflectional-wave interactions with weakly three-dimensional input
}

S. N. TIMOSHIN and F. T. SMITH

Journal of Fluid Mechanics / Volume 348 / October 1997, pp 247 - 294

DOI: 10.1017/S0022112097006447, Published online: 08 September 2000

Link to this article: http://journals.cambridge.org/abstract_S0022112097006447

How to cite this article:

S. N. TIMOSHIN and F. T. SMITH (1997). Vortex/inflectional-wave interactions with weakly three-dimensional input. Journal of Fluid Mechanics, 348, pp 247-294 doi:10.1017/S0022112097006447

Request Permissions : $\underline{\text { Click here }}$ 


\title{
Vortex/inflectional-wave interactions with weakly three-dimensional input
}

\author{
By S. N. TIMOSHIN AND F. T. SMITH \\ Department of Mathematics, University College London, Gower Street, \\ London WC1E 6BT, UK
}

(Received 12 June 1995 and in revised form 23 April 1997)

The subtle impact of the spanwise scaling in nonlinear interactions between oblique instability waves and the induced longitudinal vortex field is considered theoretically for the case of a Rayleigh-unstable boundary-layer flow, at large Reynolds numbers. A classification is given of various flow regimes on the basis of Reynolds-stress mechanisms of mean vorticity generation, and a connection between low-amplitude non-parallel vortex/wave interactions and less-low-amplitude non-equilibrium criticallayer flows is discussed in more detail than in previous studies. Two new regimes of vortex/wave interaction for increased spanwise lengthscales are identified and studied. In the first, with the cross-scale just slightly larger than the boundary-layer thickness, the wave modulation is governed by an amplitude equation with a convolution and an ordinary integral term present due to nonlinear contributions from all three Reynolds-stress components in the cross-momentum balance. In the second regime the cross-scale is larger, and the wave modulation is found to be governed by an integral/partial differential equation. In both cases the main-flow non-parallelism contributes significantly to the coupled wave/vortex development.

\section{Introduction}

Nonlinear three-dimensional interactions are now commonly recognized as playing a substantial role in laminar-turbulent transition in almost any high-Reynoldsnumber shear flow. Among these, an interaction between the primary input (or secondary induced) oblique waves and the induced mean vortex structures is deemed to be particularly important at later stages in transition, as observed experimentally in e.g. Klebanoff, Tidstrom \& Sargent (1962), Kachanov \& Levchenko (1984), Williams, Fasel \& Hama (1984), Williams (1987), among others. The concern of the present paper is with the mechanism of a nonlinear coupling between oblique waves and self-induced streamwise vortices (a vortex-wave interaction or VWI) in a boundary-layer-type flow supporting Rayleigh instability modes. The key elements of the process, namely a rapid development of the wave-induced three-dimensional corrections to the mean-flow profile which, in turn, alter the growth rate of the input waves (or, alternatively, give rise to stronger secondary instabilities) are, of course, inherent in many other interactions; see e.g. Hall \& Smith (1988, 1989, 1990, 1991), Smith \& Walton (1989), Bassom \& Hall (1990), Bennett, Hall \& Smith (1991), Smith \& Bowles (1992), Stewart \& Smith (1992), Walton \& Smith (1992), Walton, Bowles \& Smith (1994), Davis \& Smith (1994), Wu, Stewart \& Cowley (1996). What makes the physics involved different in our study is the specific role of a critical layer in the 
vortex generation considered in conjunction with the effects of cross-scaling and the main-flow non-parallelism.

Apart from a general interest in nonlinear dynamics during the latest stages of transition this study was particularly motivated by the succession of theoretical contributions in Hall \& Smith (1991), Brown et al. (1993), Smith, Brown \& Brown (1993), Brown \& Smith (1996), Allen, Brown \& Smith (1996) on VWI in inviscidly unstable flows, by Wu et al. (1996) on packets of Tollmien-Schlichting waves, and Wu (1993), Wu, Lee \& Cowley (1993) on weakly nonlinear Rayleigh waves with viscous non-equilibrium critical layers.

Studying the mechanics of a possible equilibration of instabilities in transition, Hall \& Smith (1991) suggested a scheme with a strong coupling between a largescale mean vortex field and low-amplitude short-scale inviscid inflectional (Rayleigh) disturbances. The vortex in that paper is driven by nonlinear Reynolds stresses working near the waves' critical layer, and the development of the vortex downstream must be such as to keep the wave system in saturation, i.e. in a locally neutral state, at each streamwise location. Subsequently Brown et al. (1993) demonstrated the likely start of the saturated VWI from the point of neutral linear stability for a pair of oblique fixed-frequency waves propagating in a two-dimensional inflectional boundary layer. Smith et al. (1993) found that the saturated stage is preceded by a non-saturated transient flow regime in a smaller neighbourhood of the neutral point. The non-saturated interaction is strongly influenced by the main-flow nonparallelism and the input conditions, so that the development downstream can follow various routes; these include, in addition to an asymptotic approach to saturation, a finite-distance singular breakdown, a decay, or self-sustained oscillations. Effects of non-symmetric input and weak cross-flow create even more complicated patterns including apparently chaotic responses, according to Brown \& Smith (1996), Allen et al. (1996). In all these studies the cross-scale in the flow was assumed to be of the same order of magnitude as the basic boundary-layer thickness (that is the wave inclination to the free stream is of order one).

The weakly nonlinear regimes considered in the VWI theory usually rely on minimum input amplitude sufficient to maintain interaction, in the case of Rayleigh waves in competition with the flow non-parallelism or unsteadiness. The role of the waves' critical layer is then limited to a viscous quasi-steady amplitude modulation which provides linear wave decay/growth. At the same time higher-order nonlinearities especially in the cross-momentum balance generate mean-flow corrections which spread and diffuse over a somewhat thicker buffer zone; the effect is strong and often noticeable even at moderate Reynolds numbers; see Benney \& Lin (1960). It is in the diffusion buffer where the interaction of the primary waves with the induced vortex takes place. Disturbances with sufficiently high starting level are less prone to the non-parallel effects, but instead they may become subject to stronger nonlinear interactions (still within the weakly nonlinear amplitude-modulation framework) accumulated entirely in the critical-layer zone, typically in the regime of viscous nonequilibrium critical layer first discovered by Hickernell (1984); see e.g. Stewartson (1981), Maslowe (1986) for a general review of the critical-layer theory. Hickernell's application to nominally two-dimensional Rossby waves may seem remote from the wave-wave or vortex-wave interaction problems arising in boundary-layer transition modelling, nevertheless the basic ideas leading, as in the VWI theory, to non-local integral-differential modulation equations were found to be applicable to a variety of flows containing planar and oblique isolated modes, Goldstein \& Leib (1989), Leib (1991), Wu \& Cowley (1995), pairs of oblique waves in the inviscid (Goldstein 
\& Choi 1989) and fully viscous (Wu et al. 1993) approximations, parametric resonances and nonlinear triads, Goldstein \& Lee (1992), Wu (1992), Mankbadi, Wu \& Lee (1993), Khokhlov (1994), Wu (1995), and weakly modulated wave packets, $\mathrm{Wu}(1993)$.

With regard to the main topic of this paper the work by Wu et al. (1993) and Wu (1993) on critical-layer interactions promoted by Rayleigh wave pairs and wave packets respectively is directly relevant. Wu et al. (1993) show that a non-equilibrium critical-layer flow with a two-wave interaction reduces to a parallel-flow version of the VWI in Smith et al. (1993) when the wave amplitude is made sufficiently small. The critical-layer structure in this limit splits into a viscous critical layer and a vortex diffusion layer, just as in the VWI theory. The second typical flow regime identified in $\mathrm{Wu}(1993)$ is for multi-mode interactions with larger cross-scales. The complex multiple-mode nature of the flow is reflected in the appearance of partial spanwise derivatives in the nonlinear term of the controlling amplitude equation (an example of the cross-flow instability exhibiting similar properties is given in Gajjar 1996). In the low-amplitude limit a VWI-type splitting of the critical layer occurs, and an amplitude equation of a VWI-type emerges again, but with a nonlinear convolution integral different from that in Smith et al. (1993) or Wu et al. (1993). The reason for this difference was uncovered in Timoshin \& Smith (1993): it turns out that non-parallel low-amplitude VWI has three distinct regimes, two of them with the cross-scale greater than the typical boundary-layer thickness. The current paper presents a detailed analysis of the two new regimes: the larger-scale interaction corresponding to Wu's (1993) critical-layer flow, and a new one on the intermediate cross-scale representing properties of both larger-scale and shorter-scale VWI. Both novel regimes are likely to have closer links with applications, for the transition often starts from nearly planar instabilities, the flow being significantly three-dimensional at the subsequent nonlinear stages owing to amplification of secondary modes or, as an alternative examined in this study (see also references above), due to self-induced weakly threedimensional interactions. Also, an analysis based on the Rayleigh instability of a planar boundary layer is expected to have much broader significance than one might anticipate formally. Indeed, a version of the VWI studied in Smith et al. (1993) happens to be typical for both the nonlinear Tollmien-Schlichting wave development on a pre-induced vortex field (Goldstein \& Wundrow 1995) and a nonlinear secondary instability of fully developed (for example Görtler) vortices (Blackaby \& Hall 1995). The low-amplitude case of Wu's (1993) theory developed further in this paper proves to be crucial for upper-branch Tollmien-Schlichting wave packets in the Blasius flatplate boundary layer; see Wu et al. (1996). Further links are found in the nonlinear triad interactions (e.g. Mankbadi et al. 1993); see also Goldstein (1994), Cowley \& $\mathrm{Wu}(1993)$.

For simplicity, the basic steady flow is taken to be a two-dimensional laminar boundary layer on a flat surface driven by an adverse external pressure gradient, with immediate generalizations to curved surfaces, wakes, near-wall jets and other flows exhibiting the inflectional Rayleigh kind of instability. The analysis is carried out in non-dimensional Cartesian coordinates $L_{d} x, L_{d} y, L_{d} z$ where $x$ is in the freestream direction, $y$ is normal to the surface and $z$ is along the span. The dimensional reference length $L_{d}$ is assumed comparable with the development length of the boundary layer. The wave disturbance imposed on the main steady flow consists, initially at least, of two linear Rayleigh modes of fixed equal frequency with a symmetric orientation of their wave fronts with respect to the main stream, with further generalizations made in a subsequent section. The streamwise coordinate of 
the common point of neutral linear stability for these waves is denoted by $x_{n}$. The particular source of disturbances is of little concern here; in practical situations it can be for instance a vibrating ribbon, a localized time-periodic injection/suction through the wall, inhomogeneities in the free steam, etc. The important characteristics of the wave perturbations include the frequency $U_{d} L_{d}^{-1} R^{1 / 2} \Omega$, the spanwise period $\delta z$ and the amplitude (to be introduced in the next section), where $U_{d}$ is the typical inviscid-flow velocity at the position of the neutral point and $R=U_{d} L_{d} v_{d}^{-1}$ is the Reynolds number, $v_{d}$ being the kinematic viscosity. If, further, $\left(U_{d} u, U_{d} v, U_{d} w\right), \rho_{d}, \rho_{d} U_{d}^{2} p, U_{d} L_{d}^{-1} t$ denote the velocity components in the directions $(x, y, z)$, the density, the variable part of the pressure and time respectively then the Navier-Stokes equations are written in the form

$$
\begin{gathered}
\frac{\partial u}{\partial t}+u \frac{\partial u}{\partial x}+v \frac{\partial u}{\partial y}+w \frac{\partial u}{\partial z}=-\frac{\partial p}{\partial x}+R^{-1} \nabla^{2} u \\
\frac{\partial v}{\partial t}+u \frac{\partial v}{\partial x}+v \frac{\partial v}{\partial y}+w \frac{\partial v}{\partial z}=-\frac{\partial p}{\partial y}+R^{-1} \nabla^{2} v \\
\frac{\partial w}{\partial t}+u \frac{\partial w}{\partial x}+v \frac{\partial w}{\partial y}+w \frac{\partial w}{\partial z}=-\frac{\partial p}{\partial z}+R^{-1} \nabla^{2} w \\
\frac{\partial u}{\partial x}+\frac{\partial v}{\partial y}+\frac{\partial w}{\partial z}=0
\end{gathered}
$$

with $\nabla^{2}=\partial^{2} / \partial x^{2}+\partial^{2} / \partial y^{2}+\partial^{2} / \partial z^{2}$.

In the following section the scales for the typical regimes of VWI in the physically realistic moderate-to-large range of the spanwise period $\delta z$ are deduced from a qualitative argument. It is shown that in the theory of Smith et al. (1993) only two out of three nonlinear contributions from the Reynolds stresses in the crossmomentum balance are active. The third contribution represented by the average of the term $v \partial w / \partial y$ in (1.3) produces, however, a relatively large correction to the induced vortex. This correction is justifiably small if $\delta z$ is strictly of $O\left(R^{-1 / 2}\right)$ or less, but even a small increase in the cross-scaling, from $O\left(R^{-1 / 2}\right)$ in Smith et al. (1993) to $O\left(R^{-23 / 48}\right)$, brings about an additional nonlinearity in the waveamplitude equations. These are derived in \$3. The extra contribution is of the convolution-integral form present in $\mathrm{Wu}$ (1993) and it is found to have a strong impact on the solution properties. In particular, the nonlinear oscillatory development tends to become more stable to the Hall-Smith type of saturation. This is so for symmetric two-wave configurations, whereas asymmetric input leads to a filtering phenomenon with one wave strongly inhibited in favour of the freely developing second wave. Solutions with finite-distance singularities and decaying downstream are also obtained, more in agreement with Smith et al. (1993) but in a somewhat different form. Based on the estimates of $\$ 2$, the second characteristic case of a weakly three-dimensional disturbance with $\delta z$ increased further to $O\left(R^{-3 / 8}\right)$ is considered in $\S 4$. This regime provides a VWI analogue of the $\mathrm{Wu}$ (1993) non-equilibrium critical-layer flow and at the same time a non-parallel version of the more recent theory of Wu et al. (1996). Comparison of the solution properties in the last paper with those obtained in $\S 4$ indicates a very complicated dependence of the flow evolution on the specific basic state and on the nature and character of the input perturbation. Further discussion is given in the concluding $\S 5$. 


\section{Qualitative classification of the flows with VWI}

The analysis in this section shows how order-of-magnitude estimates for various regimes of the vortex/wave coupling follow from qualitative arguments. We assume, for definiteness, that two oblique waves are induced in a Rayleigh-unstable planar boundary layer and, as they propagate downstream, the waves achieve maximum amplitudes at the neutral station $x=x_{n}$ (the estimates hold also for the case of minimum amplitudes at $x_{n}$ although the effects discussed here require then the wave generator to be located close to $x_{n}$; see $\left.\S \S 3,4\right)$. Since the Reynolds number is large, the wave motion near $x_{n}$ has two distinct lengthscales in the main-flow direction: the wavelength of neutral oscillations, of order $R^{-1 / 2}$, and a somewhat larger scale, say $\delta x$, for wave modulation due to the main-flow non-parallelism. An estimate for $\delta x$ will be given later. Another spatial characteristic, the spanwise period of the flow, $\delta z$, depends on the particular disturbance source and can be regarded as a free parameter. Thus the relations

$$
x-x_{n}=R^{-1 / 2} X=(\delta x) x_{1}, \quad z=(\delta z) Z, \quad t=R^{-1 / 2} T
$$

give appropriate scaled local variables for the system of oblique Rayleigh waves.

The flow regimes considered in the VWI theory are dominated by two physical processes. First, the nonlinear Reynolds-stress terms in the momentum balances create small three-dimensional mean corrections to the primary two-dimensional velocity field, with a particularly pronounced streamwise vorticity component. Second, the wave evolution becomes affected by the induced mean flow. The two-way interactive balancing is such that the wave modulation reinforces (or inhibits) the vortex production, and vice versa; however, in an order-of-magnitude analysis it proves convenient to first treat the two processes as unrelated, independent also of the non-parallel effects.

We start with the mean-vortex generation near the waves' neutral point at $x_{n}$. Let $A(\ll 1)$ denote the typical wave amplitude. In the bulk of the boundary layer, where $\bar{y}=y R^{1 / 2}$ is $O(1)$, the wave-perturbed velocities and pressure have the following structure:

$$
\begin{gathered}
u=U_{0}(\bar{y})+(\delta x) x_{1} U_{1}(\bar{y})+\ldots+A\left[E u^{(0)}+(\delta x) E u^{(1)}+R^{-1}(\delta z)^{-2} E u^{(2)}+\ldots\right] \\
v=\ldots+A\left[E v^{(0)}+(\delta x) E v^{(1)}+R^{-1}(\delta z)^{-2} E v^{(2)}+\ldots\right] \\
w=A R^{-1 / 2}(\delta z)^{-1} w^{(0)}+\ldots \\
p=\ldots+A\left[E p^{(0)}+(\delta x) E p^{(1)}+R^{-1}(\delta z)^{-2} E p^{(2)}+\ldots\right] .
\end{gathered}
$$

Here $U_{0}(\bar{y})$ is the basic-state profile at $x_{n}$. The next term in (2.2) is due to the main-flow non-parallelism. The wave terms are marked with the factor $E=\exp [\mathrm{i}(\alpha X-\Omega T)]$ composed of the fast variables with the real wavenumber $\alpha$ and frequency $\Omega$. The case of a monotonic inflectional profile $U_{0}(\bar{y})$ is taken for simplicity (as, for instance, in a decelerating boundary layer prior to separation), hence a single critical level, at $\bar{y}=\bar{y}_{c}$ say, exists typically such that $U_{0}\left(\bar{y}_{c}\right)=\Omega / \alpha=c$, where $c$ is the disturbance phase speed, and $U_{0}^{\prime \prime}\left(\bar{y}_{c}\right)=0$, in accordance with Rayleigh's criterion. If, in addition, $\delta z \gg R^{-1 / 2}$ then the leading-order wave terms $u^{(0)}, v^{(0)}, p^{(0)}$ are essentially quasitwo-dimensional and therefore regular at the critical level. The next-order terms $u^{(1)}, v^{(1)}, p^{(1)}$ appear in response to the flow non-parallelism and their nature is also predominantly two-dimensional. By contrast, the terms $w^{(0)}, u^{(2)}, v^{(2)}, p^{(2)}$ result from 
the wave obliqueness. The inviscid balancing in (1.3) then yields

$$
\mathrm{i} \alpha\left(U_{0}-c\right) w^{(0)}+\frac{\partial p^{(0)}}{\partial Z}=0,
$$

and hence $w^{(0)}$ and, from (1.4), $u^{(2)}$ are singular at the critical level,

$$
w^{(0)}=O\left(\left(\bar{y}-\bar{y}_{c}\right)^{-1}\right), \quad u^{(2)}=O\left(\left(\bar{y}-\bar{y}_{c}\right)^{-1}\right), \quad \text { as } \quad \bar{y} \rightarrow \bar{y}_{c},
$$

whilst $v^{(2)}$ and $p^{(2)}$ remain bounded, cf. Benney (1961). These singularities are smoothed out in the viscous critical layer where $y_{1}=R^{1 / 6}\left(\bar{y}-\bar{y}_{c}\right)$ is of $O(1)$ and the flow functions expand in the form

$$
\begin{gathered}
u=c+R^{-1 / 6} U_{0}^{\prime}\left(\bar{y}_{c}\right) y_{1}+\ldots+A\left[E \tilde{u}_{0}+(\delta x) E \tilde{u}_{1}+R^{-5 / 6}(\delta z)^{-2} E \tilde{u}_{2}+\ldots\right], \\
v=\ldots+A\left[E \tilde{v}_{0}+\ldots\right], w=A\left[R^{-2 / 6}(\delta z)^{-1} E \tilde{w}_{0}+\ldots\right], \\
p=\ldots+A\left[E \tilde{p}_{0}+\ldots\right] .
\end{gathered}
$$

The first two terms in (2.8) represent the base-flow profile. The main wave terms $\tilde{u}_{0}, \tilde{v}_{0}, \tilde{p}_{0}$ are independent of $y_{1}$ and follow on setting $\bar{y}=\bar{y}_{c}$ in the leading wave terms of (2.2), (2.3), (2.5). The $y_{1}$-dependence of $\tilde{w}_{0}, \tilde{u}_{2}$ is less trivial, for above and below the critical layer they must match with $(2.7)$. Hence $\left(\tilde{w}_{0}, \tilde{u}_{2}\right)=O\left(y_{1}^{-1}\right)$ as $y_{1} \rightarrow \pm \infty$, that is the wave oscillations are strongest inside the critical layer. This is where the cross-flow Reynolds stresses give rise to relatively strong mean flow. With the subscript $m$ denoting the average over the time period we obtain

$$
\begin{aligned}
R^{2 / 6} \frac{\partial^{2} w_{m}}{\partial y_{1}^{2}}=A^{2} R^{-2 / 3}(\delta z)^{-3}\left(\mathrm{i} \alpha \tilde{u}_{2}^{*} \tilde{w}_{0}+\tilde{w}_{0}^{*} \frac{\partial \tilde{w}_{0}}{\partial Z}+\text { c.c. }\right) \\
+A^{2} R^{2 / 6}(\delta z)^{-1}\left(\tilde{v}_{0}^{*} \frac{\partial \tilde{w}_{0}}{\partial y_{1}}+\text { c.c. }\right)+\ldots,
\end{aligned}
$$

from (1.3). Here the terms in the right-hand side represent the chief contributions from the derivatives $u \partial w / \partial x, w \partial w / \partial z, v \partial w / \partial y$ respectively, and * and c.c. denote the complex conjugate.

The wave-induced mean flow does not remain confined to the interior of the critical layer, for (2.11) suggests that

$$
w_{m}=A^{2} R^{-1}(\delta z)^{-3}\left(I_{ \pm} y_{1}+\ldots\right)+A^{2}(\delta z)^{-1} J_{ \pm}+\ldots \text { as } y_{1} \rightarrow \pm \infty,
$$

with the $y_{1}$-independent coefficients $I_{+}, J_{+}$representing integrated effects of the Reynolds stresses. In general $I_{+} \neq I_{-}$and $J_{+} \neq J_{-}$. Note that the cross-shear discontinuity (the first term in (2.12)) comes from the time-average $\langle u \partial w / \partial x+w \partial w / \partial z\rangle$, whereas the velocity jump is due to $\langle v \partial w / \partial y\rangle$. The sketches in figure $1(a)$ and $1(b)$ illustrate qualitatively the induced cross-flow velocity near the critical layer.

Physically, the induced cross-velocity jumps act as local sources of streamwise vorticity distributed over the critical-layer surface at $y=R^{-1 / 2} \bar{y}_{c}$. The vorticity diffuses in the direction normal to the critical layer and advects downstream, thereby rearranging the mean field in the primary two-dimensional flow. At large Reynolds numbers, the vortex diffusion/advection stays localized in a thin layer in the middle of the flow. The streamwise lengthscale $\delta x_{v}$ of this so-called diffusion or buffer layer depends on the particular flow regime. For instance, in a main flow with negligible or artificially suppressed non-parallelism the value of $\delta x_{v}$ can become comparable with the development length of the main flow, see Hall \& Smith (1991), Brown et al. (1993). Using, as before, the subscript $m$ for the induced mean flow we have the following 

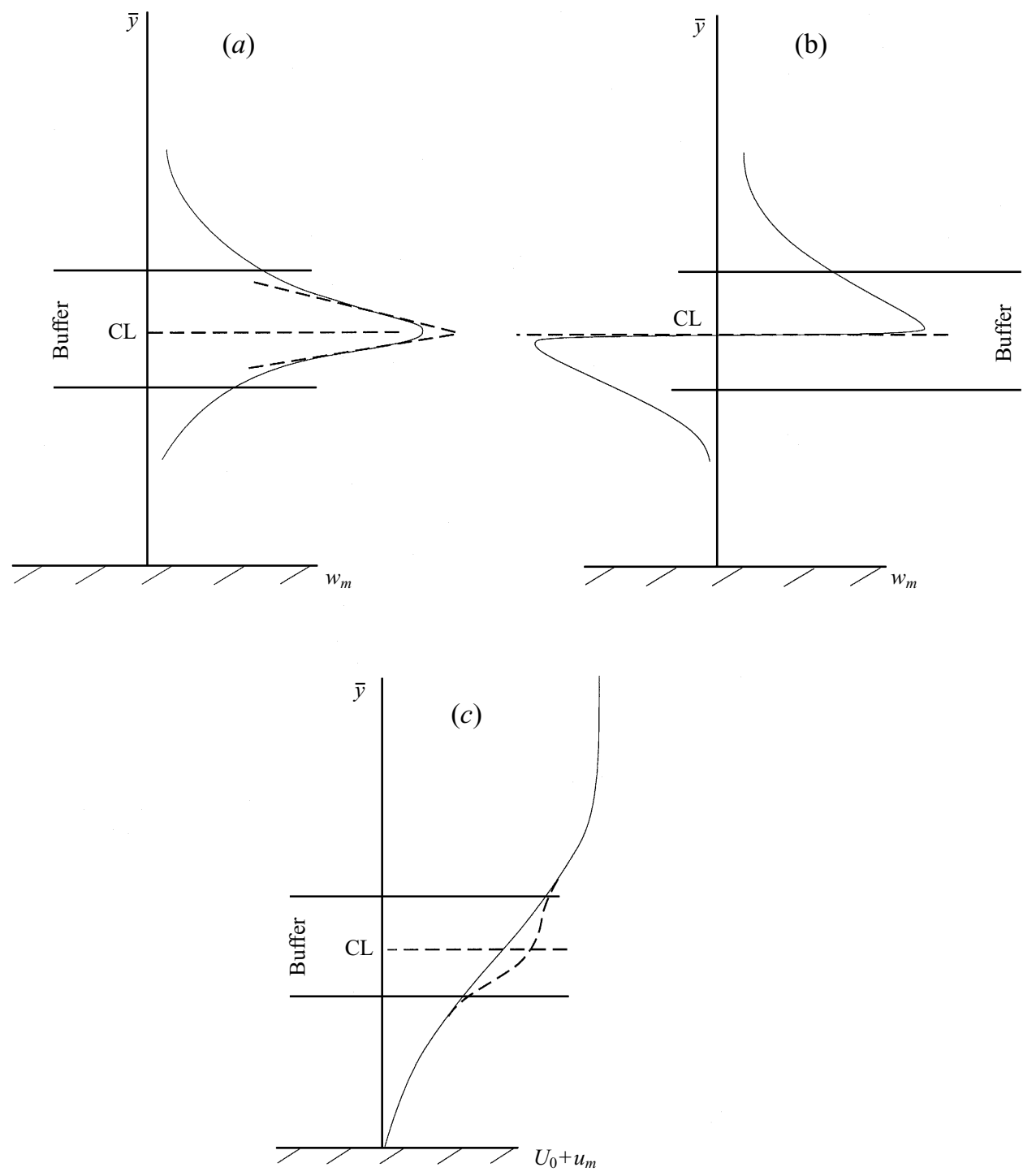

FIGURE 1. The induced mean flow in the buffer region: $(a, b)$ two contributions to the cross-velocity $w_{m}$ induced by different groups of the Reynolds stresses; $(c)$ mean corrections $u_{m}$ to the unperturbed streamwise velocity profile $U_{0}$.

main-order momentum and mass-conservation balancing for the longitudinal and cross-flow components in the buffer:

$$
\begin{gathered}
U_{0}\left(\bar{y}_{c}\right) \frac{\partial u_{m}}{\partial x}+R^{1 / 2} v_{m} U_{0}^{\prime}\left(\bar{y}_{c}\right)=R^{-1} \frac{\partial^{2} u_{m}}{\partial y^{2}}+\ldots, \\
U_{0}\left(\bar{y}_{c}\right) \frac{\partial w_{m}}{\partial x}=R^{-1} \frac{\partial^{2} w_{m}}{\partial y^{2}}+\ldots, \frac{\partial v_{m}}{\partial y}+\frac{\partial w_{m}}{\partial z}+\ldots=0 .
\end{gathered}
$$

In consequence the thickness of the buffer layer is evaluated as

$$
y-\bar{y}_{c} R^{-1 / 2}=O\left(\left(\delta x_{v}\right)^{1 / 2} R^{-1 / 2}\right),
$$


and then

$$
\begin{gathered}
w_{m}=O\left(A^{2} R^{-5 / 6}(\delta z)^{-3}\left(\delta x_{v}\right)^{1 / 2}\right)_{I}+O\left(A^{2}(\delta z)^{-1}\right)_{J}, \\
u_{m}=O\left(A^{2} R^{-5 / 6}(\delta z)^{-4}\left(\delta x_{v}\right)^{2}\right)_{I}+O\left(A^{2}(\delta z)^{-2}\left(\delta x_{v}\right)^{3 / 2}\right)_{J},
\end{gathered}
$$

on account of (2.12), where the subscripts $I, J$ refer to the corresponding jump values. The larger of the two terms in (2.17) provides the estimate for the wave-induced streamwise velocity in the buffer.

Consider now the impact of the induced mean flow on the wave evolution. Figure 1(c) shows qualitatively the effect of the vortex on the flow profile. Since the primary waves are neutral with respect to the basic-state profile $U_{0}(\bar{y})$ small changes in the mean flow make the waves slightly decaying or growing. An estimate for the growthrate correction can be obtained from the following argument. Let $\alpha, \bar{\beta}$ denote the normalized $x-, z$-wavenumbers of the oblique waves referred to the boundary-layer thickness. The wave stability is determined by the Rayleigh equation

$$
(U-c)\left(\frac{\mathrm{d}^{2} \varphi}{\mathrm{d} \bar{y}^{2}}-\left(\alpha^{2}+\bar{\beta}^{2}\right) \varphi\right)=\frac{\mathrm{d}^{2} U}{\mathrm{~d} \bar{y}^{2}} \varphi,
$$

where $U(\bar{y})=U_{0}(\bar{y})+u_{m}$ designates the new perturbed velocity profile, and for a given $\bar{\beta}$ the wavenumber $\alpha=\alpha_{0}$ is real if $u_{m} \equiv 0$. In accordance with (2.15), the mean-velocity correction is centred in a layer of thickness $\bar{y}-\bar{y}_{c}=O\left(\left(\delta x_{v}\right)^{1 / 2}\right)$ near the inflection point $\bar{y}_{c}$. Hence $\alpha=\alpha_{0}+\delta \alpha$, where the estimate

$$
\delta \alpha=O\left(u_{m}\left(\bar{y}-\bar{y}_{c}\right)^{-2}\right)=O\left(u_{m}\left(\delta x_{v}\right)^{-1}\right)
$$

follows readily from analysis of the Rayleigh problem in the domains $\bar{y}-\bar{y}_{c}=O(1)$ and $\bar{y}-\bar{y}_{c}=O\left(\left(\delta x_{v}\right)^{1 / 2}\right)$. We conclude that the length of the wave-modulation interval, say $\delta x_{w}$, due to the induced vortex is estimated as

$$
\delta x_{w}=O\left(R^{1 / 3} A^{-2}(\delta z)^{4}\left(\delta x_{v}\right)^{-1}\right)_{I} \quad \text { or } \quad O\left(R^{-1 / 2} A^{-2}(\delta z)^{2}\left(\delta x_{v}\right)^{-1 / 2}\right)_{J},
$$

where the dominant term is whichever is the smaller one.

To summarize so far, we have three typical lengths associated with different processes: the non-parallel modulation lengthscale $\delta x$, the vortex-flow scale $\delta x_{v}$, and the vortex-induced modulation lengthscale $\delta x_{w}$. Consider now various regimes of the wave motion starting with low-level input, and then gradually increasing the disturbance amplitudes. The development of infinitesimal waves depends entirely on the flow non-parallelism, with the ensuing typical length $\delta x=O\left(R^{-1 / 4}\right)$. This can be seen from the WKB-type wave solution $\exp \left[\mathrm{i} Q(x) R^{1 / 2}-\Omega T\right]$, expanding the (complex) phase speed in the neighbourhood of the neutral point $x=x_{n}$, where $Q^{\prime}\left(x_{n}\right)$ is real. Alternatively the first equality in (2.19) can be used in conjunction with the estimate, $u_{m}=O\left(\left(x-x_{n}\right)\left(\bar{y}-\bar{y}_{c}\right)^{2}\right)$, for the non-parallel curvature term in the basic-state boundary layer. The non-parallel wave decay prevents the vortex from being generated over larger $x$-intervals; therefore

$$
\delta x_{v}=\delta x=O\left(R^{-1 / 4}\right) .
$$

For such tiny disturbances the effect of the induced vortex can be neglected. As the input amplitude increases, however, the VWI first comes into operation when

$$
\min \left\{O\left(R^{7 / 12} A^{-2}(\delta z)^{4}\right)_{I}, \quad O\left(R^{-3 / 8} A^{-2}(\delta z)^{2}\right)_{J}\right\}=O\left(R^{-1 / 4}\right),
$$

on account of (2.20), (2.21). If, in particular, $\delta z=R^{-1 / 2}$, then the cross-velocity jump at the critical level, i.e. the $J$-term in (2.22), is insignificant and we have $A=O\left(R^{-7 / 12}\right)$. This flow regime is exactly that studied in Smith et al. (1993). 
The relation (2.22) suggests a new form of VWI, however, when the spanwise scale of the disturbances is just a little larger than the boundary-layer thickness. For the $I$ and $J$-terms have the same order of magnitude if

$$
\delta z=O\left(R^{-23 / 48}\right), \quad A=O\left(R^{-13 / 24}\right) .
$$

The corresponding flow will be considered in detail in $\S 3$ of this paper.

With still larger $\delta z$ the VWI is controlled solely by the production inside the critical layer of the cross-shear discontinuity, that is by the $J$-term in (2.22), so the flow can be treated as a special version of that with the scaling (2.23). There exists, however, a distinct upper limit on the admissible $\delta z$-scale. For, from (2.18), (2.19), small changes in the $x$-wavenumber become comparable with the spanwise wave modulation when $\delta \alpha$ is $O\left(\bar{\beta}^{2}\right)$, or if $(\delta z)^{2}=O\left(\delta x_{w} R^{-1 / 2}\right)$. Using $\delta x_{w}=O\left(R^{-1 / 4}\right)$ this gives the estimates

$$
\delta z=O\left(R^{-3 / 8}\right), \quad A=O\left(R^{-7 / 16}\right),
$$

for the typical spanwise scale and the wave amplitude, the latter on account of (2.22). An analysis in $\S 4$ shows that the amplitude equation for this second new case (2.24) contains partial derivatives with respect to both $z$ - and slow $x$-variables (as in $\mathrm{Wu}$ 1993; Wu et al. 1996), in contrast with the simple mode structure of the VWI with shorter $z$-modulations in $\S 3$ and in many previous studies.

Overall, the spanwise scalings assumed in Smith et al. (1993) and given by (2.23), (2.24) mark the three typical forms of the low-amplitude, nonlinear and non-parallel flow with VWI.

Similar considerations apply to regimes with stronger input disturbances, except that the main-flow non-parallelism becomes insignificant. Indeed, the lengthscale (2.20) decreases with increasing $A$, hence $\delta x_{v}=O\left(\delta x_{w}\right)$, with $\delta x_{w}$ given by (2.20). Qualitatively different flow regimes are obtained then when simultaneously with the streamwise shortening the buffer layer becomes sufficiently thin and merges with the viscous critical layer. This takes place when $\delta x_{v}=O\left(R^{-1 / 3}\right)$, and then the estimates (2.20) lead, as above, to the classification of the typical cases in accordance with the amplitude dependence on the spanwise scale:

$$
\begin{gathered}
\delta z=O\left(R^{-1 / 2}\right), \quad A=O\left(R^{-1 / 2}\right), \\
\delta z=O\left(R^{-5 / 12}\right), \quad A=O\left(R^{-5 / 12}\right) .
\end{gathered}
$$

Now we have only two possibilities, both studied in the theory of non-equilibrium critical layers. The first option (2.25), considered in Wu et al. (1993), yields a rather general situation with all the spanwise components of the Reynolds stresses participating in the wave modulation. The case (2.26) is studied in Wu (1993). With even stronger input the perturbed flow becomes inviscid in effect, and hence an alternative system of estimates is called for; see Goldstein \& Choi (1989).

The large-scale VWI considered in Hall \& Smith (1991) requires the neutrality of the waves to be maintained in a fully non-parallel basic flow by means of a carefully correlated vortex development. Broadly speaking, the non-parallel component must be 'suppressed' by the vortex in the buffer. Putting $x-x_{n}=O\left(\delta x_{v}\right), \bar{y}-\bar{y}_{c}=O\left(\left(\delta x_{v}\right)^{1 / 2}\right)$ and $\delta z=R^{-1 / 2}$ we obtain $A=O\left(R^{-7 / 12}\right)$ regardless of the length of the interaction region, cf. Brown et al. (1993). Similar estimates for saturated regimes with larger $\delta z$-scales can be derived making use of both terms in (2.17). 


\section{Moderately weak input three-dimensionality}

In this section the VWI characterized by the scalings (2.1), (2.23) is considered in detail. The order-one fast $(X, Z, T)$ and the slow $\left(x_{1}, z_{1}, t_{1}\right)$ variables are introduced by the relations

$$
x-x_{n}=\varepsilon^{24} X=\varepsilon^{12} x_{1}, \quad z=\varepsilon^{23} Z=\varepsilon^{13} z_{1}, \quad t=\varepsilon^{24} T=\varepsilon^{12} t_{1},
$$

where $\varepsilon=R^{-1 / 48}$ is an appropriate small parameter. The main elements of the asymptotic splitting of the flow field across the boundary layer were introduced in the previous section. In the subsequent analysis we replace the tentative representations (2.2)-(2.5) and onwards by rigorous asymptotic expansions. The notation below may be regarded as independent of that in $\$ 2$.

\subsection{The main part of the boundary layer}

In the core of the flow where the normal coordinate $\bar{y}=y \varepsilon^{-24}$ is of $O(1)$ the solution of the Navier-Stokes equations expands in the form

$$
\begin{gathered}
u=U_{0}(\bar{y})+\varepsilon^{12} x_{1} U_{1}(\bar{y})+\varepsilon^{24} \frac{1}{2} x_{1}^{2} U_{2}(\bar{y})+\varepsilon^{36} \frac{1}{6} x_{1}^{3} U_{3}(\bar{y})+\ldots \\
+\varepsilon^{26}\left\{E\left[\sum_{K=0}^{5} \varepsilon^{2 K} u^{(K)}+\varepsilon^{12} u^{(6)}+\ldots\right]+\text { c.c. }\right\} \\
v=\varepsilon^{24} V_{1}(\bar{y})+\varepsilon^{36} V_{2}(\bar{y})+\ldots+\varepsilon^{26}\left\{E\left[\sum_{K=0}^{5} \varepsilon^{2 K} v^{(K)}+\varepsilon^{12} v^{(6)}+\ldots\right]+\text { c.c. }\right\} \\
w=\varepsilon^{27}\left\{E\left[\sum_{K=0}^{4} \varepsilon^{2 K} w^{(K)}+\varepsilon^{10} w^{(5)}+\ldots\right]+\text { c.c. }\right\} \\
p=p_{0}+\varepsilon^{12} x_{1} p_{1}+\varepsilon^{24} \frac{1}{2} x_{1}^{2} p_{2}+\varepsilon^{36} \frac{1}{6} x_{1}^{3} p_{3}+\ldots \\
+\varepsilon^{26}\left\{E\left[\sum_{K=0}^{5} \varepsilon^{2 K} p^{(K)}+\varepsilon^{12} p^{(6)}+\ldots\right]+\text { c.c. }\right\} .
\end{gathered}
$$

The time-independent terms $U_{0}, U_{1} \ldots, V_{0}, V_{1}, \ldots, p_{0}, p_{1} \ldots$ stem from the Taylorseries expansions of the basic-state two-dimensional steady boundary-layer solution. The wave terms contain the factor $E=\exp [\mathrm{i}(\alpha X-\Omega T)]$ with the real wavenumber $\alpha=\alpha_{0}+\varepsilon^{2} \alpha_{1}+\varepsilon^{4} \alpha_{2}+\ldots$, and frequency $\Omega=\Omega_{0}+\varepsilon^{2} \Omega_{1}+\varepsilon^{4} \Omega_{2}+\ldots$. Multiple harmonics $E^{2}, E^{3}, \ldots$ which also appear in higher approximations in (3.2)-(3.5) do not contribute to the VWI and, consequently, are not shown.

The appropriate neutral-wave solution for the leading-order disturbance can be written in the form

$$
\begin{gathered}
u^{(0)}=r b_{1}^{-1} \varphi^{\prime}(\bar{y}), v^{(0)}=-\mathrm{i} \alpha_{0} r b_{1}^{-1} \varphi(\bar{y}), \\
w^{(0)}=\frac{\mathrm{i}}{\alpha_{0} b_{1}} \frac{\partial r}{\partial Z}\left(U_{0}-c_{0}\right)^{-1}\left[U_{0}^{\prime} \varphi-\left(U_{0}-c_{0}\right) \varphi^{\prime}\right], \\
p^{(0)}=r b_{1}^{-1}\left[U_{0}^{\prime} \varphi-\left(U_{0}-c_{0}\right) \varphi^{\prime}\right], c_{0}=\Omega_{0} / \alpha_{0},
\end{gathered}
$$

where $\varphi(\bar{y})$ is the normalized solution of the Rayleigh problem

$$
\left(U_{0}-c_{0}\right)\left(\varphi^{\prime \prime}-\alpha_{0}^{2} \varphi\right)=U_{0}^{\prime \prime} \varphi, \varphi(0)=\varphi(\infty)=0, \varphi\left(\bar{y}_{c}\right)=1,
$$


with $U_{0}\left(\bar{y}_{c}\right)=c_{0}, \quad U_{0}^{\prime \prime}\left(\bar{y}_{c}\right)=0, b_{1}=U_{0}^{\prime}\left(\bar{y}_{c}\right)$. The amplitude function $r=r\left(x_{1}, z_{1}, t_{1}, Z\right)$ corresponds to the wave pressure at the critical level $\bar{y}_{c}$.

The leading-order solution above does not impose any restrictions on the fast $Z$ dependence in the wave; however, the problem solvability in the next approximation is found to be equivalent to a $Z$-periodicity, hence $\partial^{2} r / \partial Z^{2}=-\beta^{2} r$, with a real $\beta$. This and other properties of the terms under the summation signs in the expansions are discussed in Appendix A.

The wave terms $u^{(6)}, v^{(6)}, p^{(6)}$ are the first in the expansions to respond to the mainflow non-parallelism and, through the subsequent match with the inner regions, to the nonlinear effects. Omitting a number of passive inhomogeneous contributions, the normal velocity $v^{(6)}$ is found to be governed by the forced Rayleigh equation

$$
\left(U_{0}-c_{0}\right)\left(\frac{\partial^{2} v^{(6)}}{\partial \bar{y}^{2}}-\alpha_{0}^{2} v^{(6)}\right)-U_{0}^{\prime \prime} v^{(6)}=S_{1} \frac{\partial r}{\partial x_{1}}+S_{2} \mathrm{i} x_{1} r+S_{3} \mathrm{i} \frac{\partial^{2} r}{\partial z_{1} \partial Z}+S_{4} \frac{\partial r}{\partial t_{1}},
$$

where

$$
\begin{gathered}
S_{1}=b_{1}^{-1}\left[c_{0} \varphi^{\prime \prime}-\alpha_{0}^{2}\left(2 U_{0}-c_{0}\right) \varphi\right], S_{3}=\alpha_{0} b_{1}^{-1}\left(U_{0}-c_{0}\right) \varphi, \\
S_{2}=-\alpha_{0} b_{1}^{-1}\left[U_{1}^{\prime \prime} \varphi-U_{1}\left(\varphi^{\prime \prime}-\alpha_{0}^{2} \varphi\right)\right], S_{4}=b_{1}^{-1} U_{0}^{\prime \prime} \varphi\left(U_{0}-c_{0}\right)^{-1} .
\end{gathered}
$$

Additional forcing is present in a non-trivial wall condition stemming from the displacement effect of a near-wall viscous Stokes layer, namely

$$
v^{(6)}(\bar{y}=0)=\mathrm{i} \alpha_{0} b_{1}^{-1} \varphi^{\prime}(0)\left(-\mathrm{i} \alpha_{0} c_{0}\right)^{-1 / 2} r,
$$

where $\left|\arg \left(-\mathrm{i} \alpha_{0} c_{0}\right)\right|<\pi$; see e.g. Smith et al. (1993). The condition at the outer edge of the boundary layer remains trivial, $v^{(6)} \rightarrow 0$ as $\bar{y} \rightarrow \infty$.

Near the inflection point the unperturbed velocity components expand in power series of the form

$$
\begin{gathered}
U_{0}=c_{0}+b_{1}\left(\bar{y}-\bar{y}_{c}\right)+\frac{1}{6} b_{3}\left(\bar{y}-\bar{y}_{c}\right)^{3}+\ldots, \\
U_{1}=d_{0}+d_{1}\left(\bar{y}-\bar{y}_{c}\right)+\frac{1}{2} d_{2}\left(\bar{y}-\bar{y}_{c}\right)^{2}+\ldots .
\end{gathered}
$$

It is easy to show then that $v^{(6)}(\bar{y})$ is singular at the critical level, so that

$$
v^{(6)}=Q_{0}+Q_{1}\left(\bar{y}-\bar{y}_{c}\right) \ln \left|\bar{y}-\bar{y}_{c}\right|+Q_{2}^{ \pm}\left(\bar{y}-\bar{y}_{c}\right)+\ldots \quad \text { as } \quad \bar{y} \rightarrow \bar{y}_{c} \pm,
$$

where the coefficient $Q_{1}$ and the jump in $\partial v^{(6)} / \partial \bar{y}$ are given by

$$
\begin{gathered}
Q_{1}=\frac{c_{0} b_{3}}{b_{1}^{3}} \frac{\partial r}{\partial x_{1}}-\mathrm{i} x_{1} \frac{\alpha_{0}}{b_{1}^{2}}\left(d_{2}-\frac{b_{3} d_{0}}{b_{1}}\right) r+\frac{b_{3}}{b_{1}^{3}} \frac{\partial r}{\partial t_{1}}, \\
Q_{2}^{+}-Q_{2}^{-}=\Delta_{1} \frac{\partial r}{\partial x_{1}}+\mathrm{i} \Delta_{3} \frac{\partial^{2} r}{\partial z_{1} \partial Z}+\Delta_{4} \frac{\partial r}{\partial t_{1}} \\
+\left\{\mathrm{i} \alpha_{0} b_{1}^{-1}\left[\varphi^{\prime}(0)\right]^{2}\left(-\mathrm{i} \alpha_{0} c_{0}\right)^{-1 / 2}+\mathrm{i} x_{1} \Delta_{2}\right\} r,
\end{gathered}
$$

with the real constants $\Delta_{m}, m=1-4$, given by the relations

$$
\Delta_{m}=-\int_{0}^{\infty} \frac{S_{m}}{U_{0}-c_{0}} \varphi \mathrm{d} \bar{y} .
$$

Here the functions $S_{m}$ are as in (3.11)-(3.12), and the finite part of the integral is taken when necessary. 


\subsection{The inner regions}

Next we examine how the singularities of the core solution are smoothed out in the inner layers near the inflection point $\bar{y}_{c}$. As indicated in $\S 2$, the first of the two inner regions to be considered is a thin buffer zone surrounding an even thinner viscous critical layer. In the buffer the appropriate $O(1)$ normal variable is $y_{1}=\varepsilon^{-6}\left(y \varepsilon^{-24}-\bar{y}_{c}\right)$, and the flow functions expand in the form

$$
\begin{aligned}
& u=c_{0}+\varepsilon^{6} b_{1} y_{1}+\varepsilon^{12} d_{0} x_{1}+\varepsilon^{18}\left(\frac{1}{6} b_{3} y_{1}^{3}+d_{1} x_{1} y_{1}\right) \\
& +\varepsilon^{24} \tilde{U}_{0}+\ldots+\varepsilon^{22}\left\{E\left[\tilde{u}_{0}+\varepsilon^{2} \tilde{u}_{1}+\ldots+\varepsilon^{16} \ln \varepsilon \tilde{u}_{8,0}+\varepsilon^{16} \tilde{u}_{8}\right]+\text { c.c. }\right\} \text {, } \\
& v=\varepsilon^{24} \tilde{V}_{0}+\varepsilon^{30} \tilde{V}_{1}+\varepsilon^{36} \tilde{V}_{2}+\ldots \\
& +\varepsilon^{26}\left\{E\left[\tilde{v}_{0}+\varepsilon^{2} \tilde{v}_{1}+\ldots+\varepsilon^{18} \ln \varepsilon \tilde{v}_{9,0}+\varepsilon^{18} \tilde{v}_{9}\right]+\text { c.c. }\right\}, \\
& w=\varepsilon^{29} \tilde{W}_{0}+\ldots+\varepsilon^{21}\left\{E\left[\tilde{w}_{0}+\varepsilon^{2} \tilde{w}_{1}+\varepsilon^{4} \tilde{w}_{2}+\ldots\right]+\text { c.c. }\right\}, \\
& p=p_{0}+\varepsilon^{12} p_{1} x_{1}+\varepsilon^{24} \frac{1}{2} p_{2} x_{1}^{2}+\ldots \\
& +\varepsilon^{26}\left\{E\left[\tilde{p}_{0}+\varepsilon^{2} p_{1}+\ldots+\varepsilon^{18} \tilde{p}_{9}+\ldots\right]+\text { c.c. }\right\} .
\end{aligned}
$$

First we notice that a number of wave terms in the expansions reproduce the outer solution of $\$ 3.1$ rewritten in terms of $y_{1}$, for example

$$
\tilde{u}_{0}=-\frac{1}{\alpha_{0}^{2} b_{1} y_{1}} \frac{\partial^{2} r}{\partial Z^{2}}, \quad \tilde{v}_{0}=-\frac{\mathrm{i} \alpha_{0}}{b_{1}} r, \quad \tilde{w}_{0}=\frac{\mathrm{i}}{\alpha_{0} b_{1} y_{1}} \frac{\partial r}{\partial Z}, \quad \tilde{p}_{0}=r .
$$

The crucial point is actually the derivation of the governing equation for $\tilde{v}_{9}$ in (3.21), as this term must match with (3.16) in the core. On neglecting a purely polynomial part, the equation for $\tilde{v}_{9}$ can be written in the form

$$
\frac{\partial^{2} \tilde{v}_{9}}{\partial y_{1}^{2}}=\frac{Q_{1}}{y_{1}}-\frac{i \alpha_{0} r}{b_{1}^{2}} \frac{1}{y_{1}} \frac{\partial^{2} \hat{U}_{0}}{\partial y_{1}^{2}},
$$

where $Q_{1}$ is given by (3.17). The vortex effect on the wave is monitored via the induced streamwise velocity defined as

$$
\hat{U}_{0}=\tilde{U}_{0}-\frac{b_{4}}{4 !} y_{1}^{4}-\frac{x_{1} d_{2}}{2} y_{1}^{2}-\frac{x_{1}^{2}}{2} U_{2}\left(\bar{y}_{c}\right) .
$$

The match with the core flow above and below the buffer requires that

$$
\left.\frac{\partial \tilde{v}_{9}}{\partial y_{1}}\right|_{y_{1} \rightarrow+\infty}-\left.\frac{\partial \tilde{v}_{9}}{\partial y_{1}}\right|_{y_{1} \rightarrow-\infty}=Q_{2}^{+}-Q_{2}^{-} .
$$

The vortex-flow generation is controlled by the equations

$$
\begin{gathered}
c_{0} \frac{\partial \hat{U}_{0}}{\partial x_{1}}+b_{1} \hat{V}_{2}=\frac{\partial^{2} \hat{U}_{0}}{\partial y_{1}^{2}}, \frac{\partial \hat{V}_{2}}{\partial y_{1}}+\frac{\partial \tilde{W}_{0}}{\partial Z}=0, \\
c_{0} \frac{\partial \tilde{W}_{0}}{\partial x_{1}}+\left(\tilde{v}_{0} \frac{\partial \tilde{w}_{0}^{*}}{\partial y_{1}}+\text { c.c. }\right)=\frac{\partial^{2} \tilde{W}_{0}}{\partial y_{1}^{2}},
\end{gathered}
$$

with the outer-edge conditions

$$
\tilde{W}_{0} \rightarrow 0, \partial \hat{U}_{0} / \partial y_{1} \rightarrow 0, \partial \hat{V}_{2} / \partial y_{1} \rightarrow 0 \text { as } y_{1} \rightarrow \pm \infty .
$$

Here the induced normal velocity in the vortex is defined by $\hat{V}_{2}=\tilde{V}_{2}-x_{1} V_{2}\left(\bar{y}_{c}\right)-$ 
$y_{1}^{2} V_{1}^{\prime \prime}\left(\bar{y}_{c}\right) / 2$, where $V_{1}, V_{2}$ are as in (3.3). In addition, an analysis in Appendix B indicates that the finite part of $\tilde{W}_{0}$ and its first derivative are discontinuous at the critical level, in particular

$$
\begin{gathered}
\tilde{W}_{0}+b_{2}^{-1} \frac{\partial}{\partial Z}\left(|r|^{2}\right) \ln \left|y_{1}\right|=\tilde{W}_{00}^{ \pm}+y_{1} \tilde{W}_{01}^{ \pm}+O\left(y_{1}^{2}\right) \text { as } y_{1} \rightarrow \pm 0, \\
\tilde{W}_{00}^{+}-\tilde{W}_{00}^{-}=J_{0}=-\frac{\mathrm{i} \pi}{b_{1}^{2}}\left(r^{*} \frac{\partial r}{\partial Z}-r \frac{\partial r^{*}}{\partial Z}\right) \operatorname{sgn}\left(\alpha_{0} b_{1}\right), \\
\tilde{W}_{01}^{+}-\tilde{W}_{01}^{-}=J_{1}=\frac{2 \pi}{\left|\alpha_{0} b_{1}\right|^{5 / 3}}\left(\frac{2}{3}\right)^{2 / 3}\left(-\frac{2}{3}\right) ! \frac{\partial}{\partial Z}\left(\left|\frac{\partial r}{\partial Z}\right|^{2}\right),
\end{gathered}
$$

whereas $\hat{U}_{0}, \partial \hat{U}_{0} / \partial y_{1}, \partial^{2} \hat{U}_{0} / \partial y_{1}^{2}$ and $\hat{V}_{0}$ must be continuous at $y_{1}=0$. The problem (3.28)-(3.33) was solved using a Fourier transform in $x_{1}$. On substituting the result into (3.25) and integrating we find that

$$
\frac{\partial \tilde{v}_{9}}{\partial y_{1}}=Q_{1} \ln \left|y_{1}\right|+A^{ \pm}+\Phi\left(y_{1}\right) \text { at } y_{1}<0 .
$$

Here $A^{ \pm}$are independent of $y_{1}$, while the properties of the function $\Phi$ are as follows. The jump at the outer edges of the buffer region is given by

$$
\Phi(+\infty)-\Phi(-\infty)=-\frac{\mathrm{i} \alpha_{0}}{2 c_{0} b_{1}} r \int_{-\infty}^{x_{1}} \frac{\partial J_{1}}{\partial Z} \mathrm{~d} x_{1}+\frac{\mathrm{i} \pi^{3 / 2} \alpha_{0}}{4 c_{0}^{1 / 2} b_{1}^{3}} r \int_{-\infty}^{x_{1}} \frac{\partial^{2}}{\partial Z}\left(|r|^{2}\right) \frac{\mathrm{d} s}{\left(x_{1}-s\right)^{1 / 2}}
$$

On approach to the critical layer $\Phi$ is singular with a continuous finite part:

$$
\Phi\left(y_{1}\right)=Q_{i} \ln \left|y_{1}\right|+O(1) ; \quad \Phi\left(y_{1}\right)-\Phi\left(-y_{1}\right) \rightarrow 0 \text { as } y_{1} \rightarrow 0,
$$

where

$$
Q_{i}=-\frac{\mathrm{i} \alpha_{0}}{4\left(\pi c_{0}\right)^{1 / 2} b_{1}} r \int_{-\infty}^{x_{1}} \frac{\partial J_{0}}{\partial Z} \frac{\mathrm{d} s}{\left(x_{1}-s\right)^{1 / 2}} .
$$

Matching (3.34)-(3.36) with the critical-layer solution of (B 8), (B 9) and satisfying the outer-edge condition (3.27) we obtain the following two relations:

$$
\begin{gathered}
A^{+}-A^{-}=\mathrm{i} \pi \operatorname{sgn}\left(\alpha_{0} b_{1}\right)\left(Q_{1}+Q_{i}\right), \\
Q_{2}^{+}-Q_{2}^{-}=\left\langle A^{+}-A^{-}+\Phi(+\infty)-\Phi(-\infty)\right\rangle .
\end{gathered}
$$

The angle brackets in (3.39) indicate that only terms proportional to $\cos \beta Z, \sin \beta Z$ are to be retained in the right-hand side.

\subsection{Equations of the VWI}

For the $Z$-periodic wave-pressure distribution we write

$$
r=R_{1}\left(x_{1}, z_{1}, t_{1}\right) \exp (\mathrm{i} \beta Z)+R_{2}\left(x_{1}, z_{1}, t_{1}\right) \exp (-\mathrm{i} \beta Z),
$$

and then the amplitude functions $R_{1}, R_{2}$ are governed by equations of the form

$$
\begin{aligned}
a \frac{\partial R_{1}}{\partial x_{1}}+\left(c x_{1}+d\right) R_{1}-\mathrm{i} \beta & \Delta_{3} \frac{\partial R_{1}}{\partial z_{1}}+a_{1} \frac{\partial R_{1}}{\partial t_{1}} \\
& =R_{2}\left[e_{0} \int_{x_{0}}^{x_{1}} R_{1} R_{2}^{*} \frac{\mathrm{d} s}{\left(x_{1}-s\right)^{1 / 2}}+e_{1} \int_{x_{0}}^{x_{1}} R_{1} R_{2}^{*} \mathrm{~d} s\right],
\end{aligned}
$$




$$
\begin{aligned}
a \frac{\partial R_{2}}{\partial x_{1}}+\left(c x_{1}+d\right) R_{2}+\mathrm{i} \beta & \Delta_{3} \frac{\partial R_{2}}{\partial z_{1}}+a_{1} \frac{\partial R_{2}}{\partial t_{1}} \\
& =R_{1}\left[e_{0} \int_{x_{0}}^{x_{1}} R_{1}^{*} R_{2} \frac{\mathrm{d} s}{\left(x_{1}-s\right)^{1 / 2}}+e_{1} \int_{x_{0}}^{x_{1}} R_{1}^{*} R_{2} \mathrm{~d} s\right],
\end{aligned}
$$

as follows from relations (3.38), (3.39) with (3.17), (3.18), (3.32), (3.33), (3.35), (3.37). The constant coefficients present are given by

$$
\begin{gathered}
a=\pi c_{0} b_{3} b_{1}^{-3} \operatorname{sgn}\left(\alpha_{0} b_{1}\right)+\mathrm{i} \Delta_{1}, \quad a_{1}=\pi b_{3} b_{1}^{-3} \operatorname{sgn}\left(\alpha_{0} b_{1}\right)+\mathrm{i} \Delta_{4}, \\
c=-\Delta_{2}-\mathrm{i} \pi \operatorname{sgn}\left(\alpha_{0} b_{1}\right) \alpha_{0} b_{3} b_{1}^{-3}\left(d_{2} b_{1} b_{3}^{-1}-d_{0}\right), \quad d=-\alpha_{0} b_{1}^{-1}\left[\varphi^{\prime}(0)\right]^{2}\left(-\mathrm{i} \alpha_{0} c_{0}\right)^{-1 / 2}, \\
e_{0}=\alpha_{0} \beta^{2} \pi^{3 / 2} c_{0}^{-1 / 2} b_{1}^{-3}, \quad e_{1}=4 \pi \alpha_{0} \beta^{4} c_{0}^{-1} b_{1}^{-1}\left|\alpha_{0} b_{1}\right|^{-5 / 3}\left(\frac{2}{3}\right)^{2 / 3}\left(-\frac{2}{3}\right) !
\end{gathered}
$$

We notice that the coefficients of the nonlinear terms are real, whereas the linear differential operators in (3.41), (3.42) contain constants which are complex in general. The nonlinearity enters through two integrals one of which is convolutionary. It is important to note that the entire calculation leading to (3.41), (3.42) holds irrespective of whether the disturbance source is situated at a finite station $x_{0}$ or the waves are coming from the infinity upstream, hence the lower limit of integration $x_{0}$ can be either negative infinite or finite. However, for the convergence of the integrals the value of $x_{0}$ must be taken finite in the case of non-parallel destabilization of the locally neutral wave (that the inviscid upper-branch neutral wave can be either stabilized or destabilized by the non-parallel effects follows from the argument given in Smith et al. 1993 and Timoshin 1996). It is also insignificant whether the bearing wave belongs to the upper neutral branch (as is typical for a decelerating boundary layer) or to an inviscid lower neutral branch as may be the case for certain non-monotonic basic-state profiles. Subtleties may arise however if the bearing neutral frequency has unstable harmonics which are almost inevitably triggered by any local wave generator placed within the VWI region; see Timoshin (1996).

With all three slow-scale derivatives present in the equations, allowance for an extra $z_{1^{-}}, t_{1}$-modulation of either of the two waves can be made easily. For instance, frequency detuning or a small difference in the wave obliqueness changes the value of the constant $d$ and therefore renders the linear operators in (3.41), (3.42) asymmetric, in the general case. However in the following analysis we set $\partial / \partial t_{1}=\partial / \partial z_{1}=0$ for simplicity, and focus on the influence of the non-parallelism introduced by the term $c x_{1}$; some of the asymmetry effects will be taken into account by allowing different input wave amplitudes.

Let us assume, to begin with, that the input amplitudes are equal, so that $R_{1}=R_{2}$. Since the wave pressure $r$ is then real the vortex/wave flow field develops in $z$ periodic strips with fixed, $x_{1}$-independent boundaries between neighbouring vortices. The system (3.41), (3.42) then reduces to the single amplitude equation

$$
\rho^{\prime}(\xi)+\tilde{c} \xi \rho=\rho\left[\tilde{e}_{0} \int_{\xi_{0}}^{\xi} \rho(s) \frac{\mathrm{d} s}{(\xi-s)^{1 / 2}}+\tilde{e}_{1} \int_{\xi_{0}}^{\xi} \rho(s) \mathrm{d} s\right],
$$

for the function $\rho=\rho_{0}^{-1}\left|R_{1}\right|^{2}=\rho_{0}^{-1}\left|R_{2}\right|^{2}, \rho_{0}$ being a characteristic value of the square of the amplitude. Here

$$
\begin{gathered}
\left(\xi, \xi_{0}\right)=|\tilde{\lambda}|^{1 / 2}\left(x_{1}, x_{0}\right)+\tilde{\mu}|\tilde{\lambda}|^{-1 / 2}, \\
\tilde{\lambda}=2\left(c_{r} a_{r}+c_{i} a_{i}\right)|a|^{-2}, \quad \tilde{\mu}=2\left(d_{r} a_{r}+d_{i} a_{i}\right)|a|^{-2},
\end{gathered}
$$




$$
\tilde{c}=\operatorname{sgn}(\tilde{\lambda}), \quad \tilde{e}_{0}=2 a_{r} e_{0}|a|^{-2}|\tilde{\lambda}|^{-3 / 4} \rho_{0}, \quad \tilde{e}_{1}=2 a_{r} e_{1}|a|^{2}|\tilde{\lambda}|^{-1} \rho_{0},
$$

and the subscripts $r, i$ refer to the real and imaginary parts respectively. A complementary equation for the phase of $R_{1}, R_{2}$ can be solved separately.

The controlling system proves to be simple enough to permit a more or less complete classification of possible solutions. As a representative example we take an initial condition in the form

$$
\rho=1 \text { at } \xi=\xi_{0}=0 .
$$

Setting $\tilde{e}_{0}=0$ we arrive then at the equation studied in Smith et al. (1993). Following their results we can expect that the particular regime of the VWI will depend primarily on the signs of the coefficients. Since $\tilde{e}_{0}, \tilde{e}_{1}$ must have the same sign, see (3.45), the following four typical cases are obtained.

(i) $\tilde{c}=1$, $\left(\tilde{e}_{0}, \tilde{e}_{1}\right)<0$. As illustrated in figures $2(a)$ and $2(b)$, both non-parallelism and nonlinearity tend to suppress the wave development. As $\xi \rightarrow \infty$ the waves decay exponentially hence the induced vortex in the buffer loses support from the Reynolds stresses and therefore develops in a passive inertia-diffusion balance. Analysis of (3.28)-(3.33) suggests that $\tilde{W}_{0}=O\left(x_{1}^{-1 / 2}\right), \hat{U}_{0}=O\left(x_{1}\right)$ at large $x_{1}$ in a viscous region of thickness $y_{1}=O\left(x_{1}^{1 / 2}\right)$. We conclude that the vortex persists and even becomes stronger (in terms of its streamwise velocity) downstream of the interaction domain, although the induced three-dimensional flow cannot compete with the non-parallel curvature effect contained in the term $x_{1} y_{1}^{2}=O\left(x_{1}^{2}\right)$ in (3.26).

(ii) $\tilde{c}=-1,\left(\tilde{e}_{0}, \tilde{e}_{1}\right)>0$. Figures $2(c)$ and $2(d)$ show how the non-parallel linear growth adds to the destabilizing nonlinearity to produce solutions terminating at a finite point $\xi=\xi_{s}$ with an unbounded amplitude growth of the form

$$
\begin{gathered}
\rho=O\left(\left(\xi_{s}-\xi\right)^{-3 / 2}\right) \text { if } \tilde{e}_{0} \neq 0, \\
\rho=O\left(\left(\xi_{s}-\xi\right)^{-2}\right) \text { if } \tilde{e}_{0}=0,
\end{gathered}
$$

from the balance between the derivative and the nonlinear terms in (3.46). Owing to the cumulative growth of both wave and vortex components the next stage in the flow development is associated with non-equilibrium effects in the viscous critical layer caused by a merging of the critical-layer domain with the buffer, cf. $\S 2$.

(iii) $\tilde{c}=1$, $\left(\tilde{e}_{0}, \tilde{e}_{1}\right)>0$. A combination of the properties in cases (i) and (ii) arises when the non-parallel decay of linear waves is linked with nonlinear destabilization, see figures $2(e)$ and $2(f)$ and Smith et al. (1993). If the values of $\tilde{e}_{0}, \tilde{e}_{1}$ are sufficiently small then the waves decay far downstream. Somewhat stronger nonlinearity causes a finite-distance breakdown as in (3.51), (3.52). The border between the two families consists of solutions with an asymptotic saturation downstream

$$
\begin{gathered}
\rho=2\left(\pi \tilde{e}_{0}\right)^{-1} \xi^{1 / 2}+\ldots \text { if } \tilde{e}_{1}=0, \\
\rho=\tilde{e}_{1}^{-1}-\tilde{e}_{0} \tilde{e}_{1}^{-2} \xi^{-1 / 2}+\ldots \text { if } \tilde{e}_{1} \neq 0,
\end{gathered}
$$

as $\xi \rightarrow \infty$. The saturation relies on a balance between the nonlinear and non-parallel terms in (3.46). Solutions in figure $2(f)$ are for a fixed $\tilde{e}_{0}$ and varying $\tilde{e}_{1}$; essentially the same behaviour is observed when $\tilde{e}_{1}$ is fixed and $\tilde{e}_{0}$ varies, and when both $\tilde{e}_{0}$ and $\tilde{e}_{1}$ vary.

(iv) $\tilde{c}=-1$, $\left(\tilde{e}_{0}, \tilde{e}_{1}\right)<0$. The equilibrium saturation was an exceptional event in the previous case due to extreme sensitivity of the flow to variations in the controlling parameters. The situation changes radically in the present case when linearly unstable 

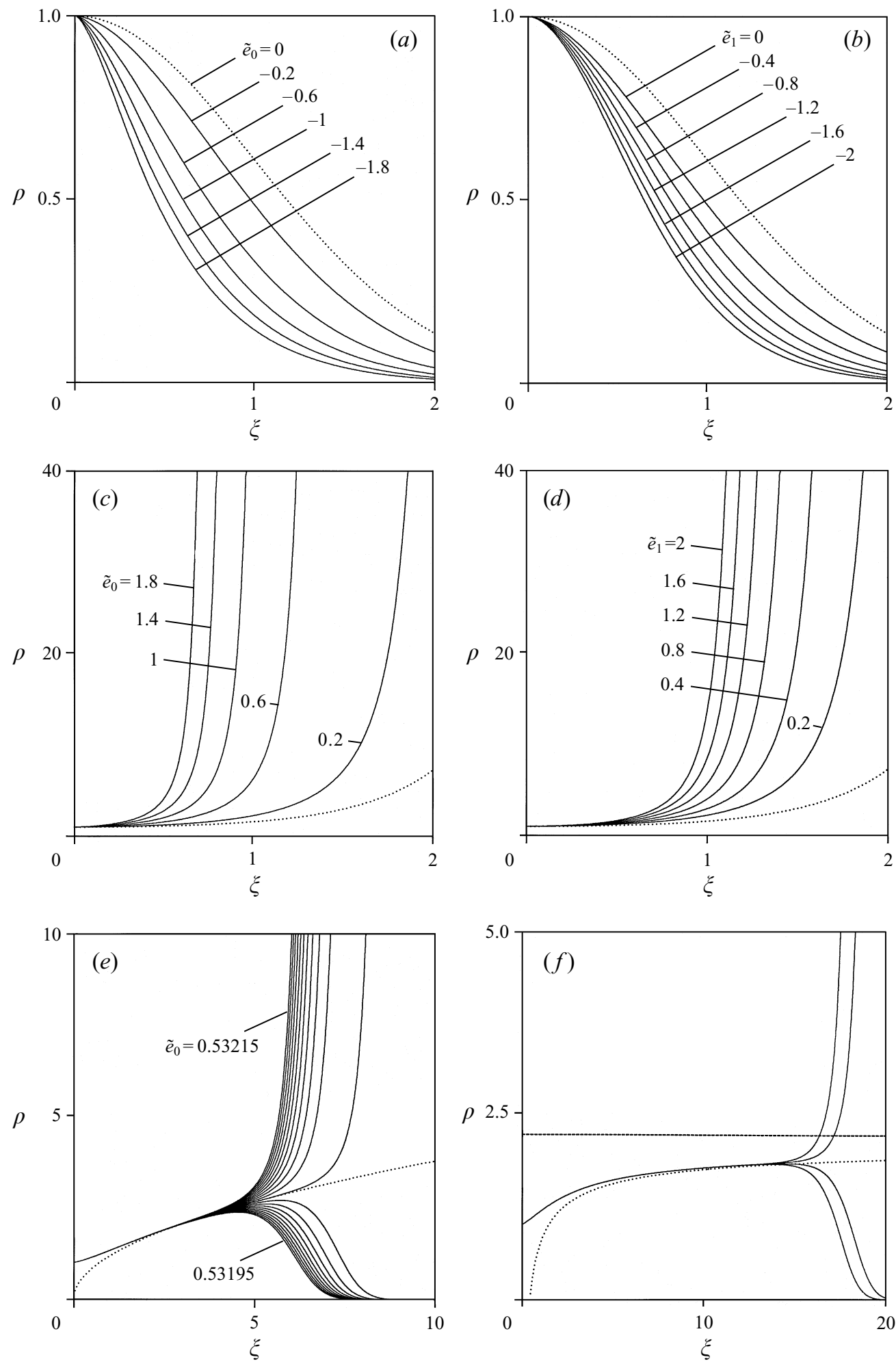

Figure $2(a-f)$. For caption see facing page. 

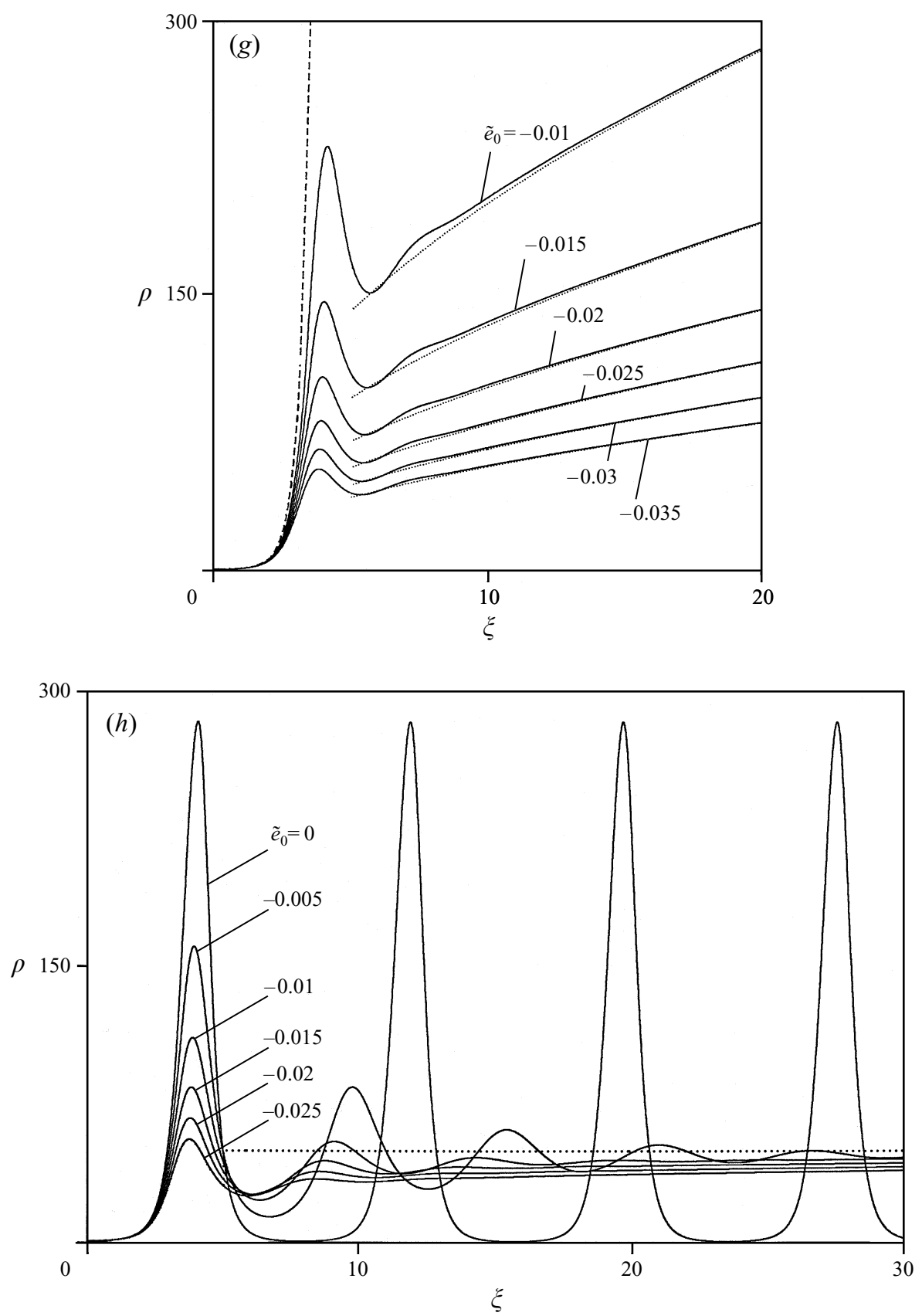

FIGURE 2. The square of the wave amplitude $\rho$ vs. the scaled coordinate $\xi$ for the VWI with equal input disturbances from the numerical solution of (3.46), (3.50). (a) $\tilde{c}=1, \tilde{e}_{1}=0 ;(b) \tilde{c}=1, \tilde{e}_{0}=-0.2$; (c) $\tilde{c}=-1, \tilde{e}_{1}=0 ;(d) \tilde{c}=-1, \tilde{e}_{0}=0.2$. The dots in $(a-d)$ show the non-parallel growth/decay of the linear waves with $\tilde{e}_{0}=\tilde{e}_{1}=0$. (e) $\tilde{c}=1, \tilde{e}_{1}=0$ : the solid lines correspond to $\tilde{e}_{0}$ varying from 0.53195 to 0.53215 with step $\Delta \tilde{e}_{0}=10^{-5} ; \cdots$, the asymptote $(3.53)$ with $\tilde{e}_{0}=0.532045 ;(f) \tilde{c}=1, \tilde{e}_{0}=0.3$ and $\tilde{e}_{1}$ varies with step $\Delta \tilde{e}_{1}=10^{-10}$ around the approximate threshold at $\tilde{e}_{1}=0.45195 \ldots ;---$ and $\cdots$ illustrate one and two terms in the asymptotic result (3.54) respectively. $(g) \tilde{c}=-1, \tilde{e}_{1}=0$; --- , linear waves; $\cdots$, the asymptote $(3.53)$. (h) $\tilde{c}=-1, \tilde{e}_{1}=-0.02 ; \cdots$, the leading term in (3.54). 
waves meet with a strongly damping nonlinearity. First of all we find that all solutions evolve towards the equilibrium state (3.53) if $\tilde{e}_{1}=0$, see figure $2(g)$. When $\tilde{e}_{1} \neq 0$ the role of the convolution integral becomes rather subtle; see figure $2(h)$. For any non-zero $\tilde{e}_{0}$ the large- $\xi$ asymptote of the solution is of the form (3.54) which, in the leading approximation, does not contain $\tilde{e}_{0}$. If, however, we put $\tilde{e}_{0}=0$ then the solution turns into a $\xi$-periodic function for all $\tilde{e}_{1}$ except $\tilde{e}_{1}=-1$, when $\rho \equiv 1$. Thus the first integral in (3.46) determines the asymptotic behaviour of the flow through a background selectivity mechanism. This is discussed in more detail in Appendix C for the case of small negative $\tilde{e}_{0}$.

The wave saturation in either of the forms (3.53) or (3.54) gives rise to strong vortex flows in the buffer with the behaviour $\tilde{W}_{0}=O\left(x_{1}^{1 / 2}\right), \hat{U}_{0}=O\left(x_{1}^{2}\right)$ when $y_{1}=O\left(x_{1}^{1 / 2}\right)$ for large $x_{1}$. Hence the induced vortex velocity compares in order of magnitude with the curvature term $x_{1} y_{1}^{2}$ in (3.26).

In the case of unequal input waves the controlling equations are (3.41), (3.42) with the initial conditions

$$
R_{1}=R_{10}, \quad R_{2}=R_{20} \text { at } x_{1}=x_{0}=0,
$$

where the constants $R_{10}, R_{20}$ are real without loss of generality. Now that the integrals become complex-valued there seems to be no simple characteristic of the effect of nonlinearity on the VWI; nevertheless we shall continue to use the signs of the products $e_{0} a_{r}, e_{1} a_{r}$ when referring to destabilizing $\left(e_{0,1} a_{r}>0\right)$ and stabilizing $\left(e_{0,1} a_{r}<0\right)$ nonlinearity. The non-parallelism stabilizes/destabilizes the waves in accordance with a positive/negative sign of $\tilde{\lambda}$ in (3.48).

Computations for a number of more or less randomly chosen controlling parameters indicate that a classification along the lines (i)-(iv) above remains applicable here. In particular, the equivalent to case (i) with $\left(e_{0} a_{r}, e_{1} a_{r}\right)<0, \tilde{\lambda}>0$, seems to always lead to ultimate decay of both waves, whereas solutions with $\left(e_{0} a_{r}, e_{1} a_{r}\right)>0, \tilde{\lambda}<0$ inevitably terminate in a finite-distance singularity. A possibility of both types of behaviour is observed in case (iii), where $\left(e_{0} a_{r}, e_{1} a_{r}\right)>0, \tilde{\lambda}>0$, see figure $3(a)$. Note that the nonlinear effects tend to eliminate the difference between the two waves in these regimes.

The last option (iv) in the case of asymmetric input provides an interesting example of the VWI with a decoupled wave development if $\tilde{\lambda}<0,\left(e_{0} a_{r}, e_{1} a_{r}\right)<0$. For a similar regime, but with $e_{0}=0$, Brown \& Smith (1996) obtained a number of periodic and quasi-periodic/irregular oscillatory solutions. With the convolution term taken into account the oscillations mostly vanish, see figures $3(b)$ and $3(c)$. Instead, the wave with the larger initial amplitude grows exponentially following the trend of a non-parallel amplification in the non-interactive linear regime. Surprisingly, the second wave becomes strongly inhibited even moderately far downstream, despite being linearly unstable. This behaviour was found to be reproducible for a wide range of input amplitudes and coefficients in the equations. The amplitude of the weaker wave performs a few sharp oscillations just before the decoupling settles down, although grid refinement did not reveal any singularities in this part of the solution. Predictions concerning the far-downstream behaviour of the decoupled waves can be made analytically. Suppose that one wave, for example $R_{1}$, decays sufficiently fast that the right-hand side in (3.42) has no impact on $R_{2}$ at leading order. Then the second wave grows as

$$
R_{2}=\text { const } \times \exp \left[-\frac{c}{2 a} x_{1}^{2}-\frac{d}{a} x_{1}\right]+\ldots
$$


with a coefficient determined by integral properties of the solution. Manipulating (3.41) we obtain that

$$
\begin{gathered}
R_{1} R_{2}^{*}=-\frac{1}{2 \pi e_{0}} \mathscr{L}_{0} x_{1}^{-3 / 2}+\ldots \text { as } x_{1} \rightarrow \infty \text { if } e_{1}=0, \\
R_{1} R_{2}^{*}=-\frac{3 e_{0}}{4 e_{1}^{2}} \mathscr{L}_{0} x_{1}^{-5 / 2}+\ldots \text { as } x_{1} \rightarrow \infty \text { if } e_{1} \neq 0,
\end{gathered}
$$

where

$$
\mathscr{L}_{0}=\int_{0}^{\infty} R_{2}^{-1}\left[a \frac{\mathrm{d} R_{1}}{\mathrm{~d} x_{1}}+\left(c x_{1}+d\right) R_{1}\right] \mathrm{d} x_{1} .
$$

Comparisons with (3.57), (3.58) are made in figures $3(b)$ and $3(c)$.

In a somewhat less spectacular form the wave decoupling is also present in the following example of the VWI with input disturbances generated far upstream of the interaction domain. On setting $x_{0}=-\infty$ in (3.41), (3.42), the initial conditions become

$$
\left(R_{1}, R_{2}\right)=\left(R_{10}, R_{20}\right) \exp \left(-\frac{c}{2 a} x_{1}^{2}-\frac{d}{a} x_{1}\right)+\ldots \text { as } x_{1} \rightarrow-\infty,
$$

with constants $R_{10}, R_{20}$. The terms explicitly shown here provide an exact solution of the linearized equations; we choose $(c / a)_{r}>0$ so that the waves decay as $x_{1} \rightarrow \pm \infty$. If in addition $\left(e_{0} a_{r}, e_{1} a_{r}\right)<0$, then the conditions in the upstream part of the flow field are similar to those for regime (iv) in that the initial linear growth combines with the nonlinear damping. Figure $3(d)$, curve 1, illustrates a strong nonlinear suppression of equal waves, whereas the main effect of unequal input is evident in the accentuated asymmetry of the wave pattern, especially at higher levels of nonlinearity, cf. curves $4,4^{\prime}$. In this case, as in figures $3(b)$ and $3(c)$, the VWI acts as a nonlinear filter which allows the initially stronger wave to develop almost freely against the background of the heavily suppressed weaker disturbance.

\section{VWI with weakly three-dimensional input}

In this section the theory is extended to flows with larger spanwise lengthscales and somewhat higher wave amplitudes, in accord with the estimates (2.24) of $\$ 2$. The derivation given in $\$ 4.1$ shows that the wave motion is governed then by a generalization of the integral partial-differential equations encountered in $\mathrm{Wu}$ (1993) and Wu et al. (1996). As in those studies, the current flow regime is subject to strong secondary instabilities and phase/amplitude exchange which, combined in our case with the main-flow non-parallelism, renders the ultimate wave development highly dependent on the controlling parameters as well as on the initial conditions, as we show in $\S 4.2$.

\subsection{Derivation of the amplitude equation.}

As in $\S 3$ we start with the flow in the core region where $\bar{y}=y R^{1 / 2}$ is of $O(1)$. In keeping with the estimates $(2.1),(2.24)$ of $\$ 2$ the scaled variables for the present interaction are introduced by

$$
x-x_{n}=\varepsilon^{8} X=\varepsilon^{4} x_{1}, \quad t=\varepsilon^{8} T, \quad z=\varepsilon^{6} Z,
$$



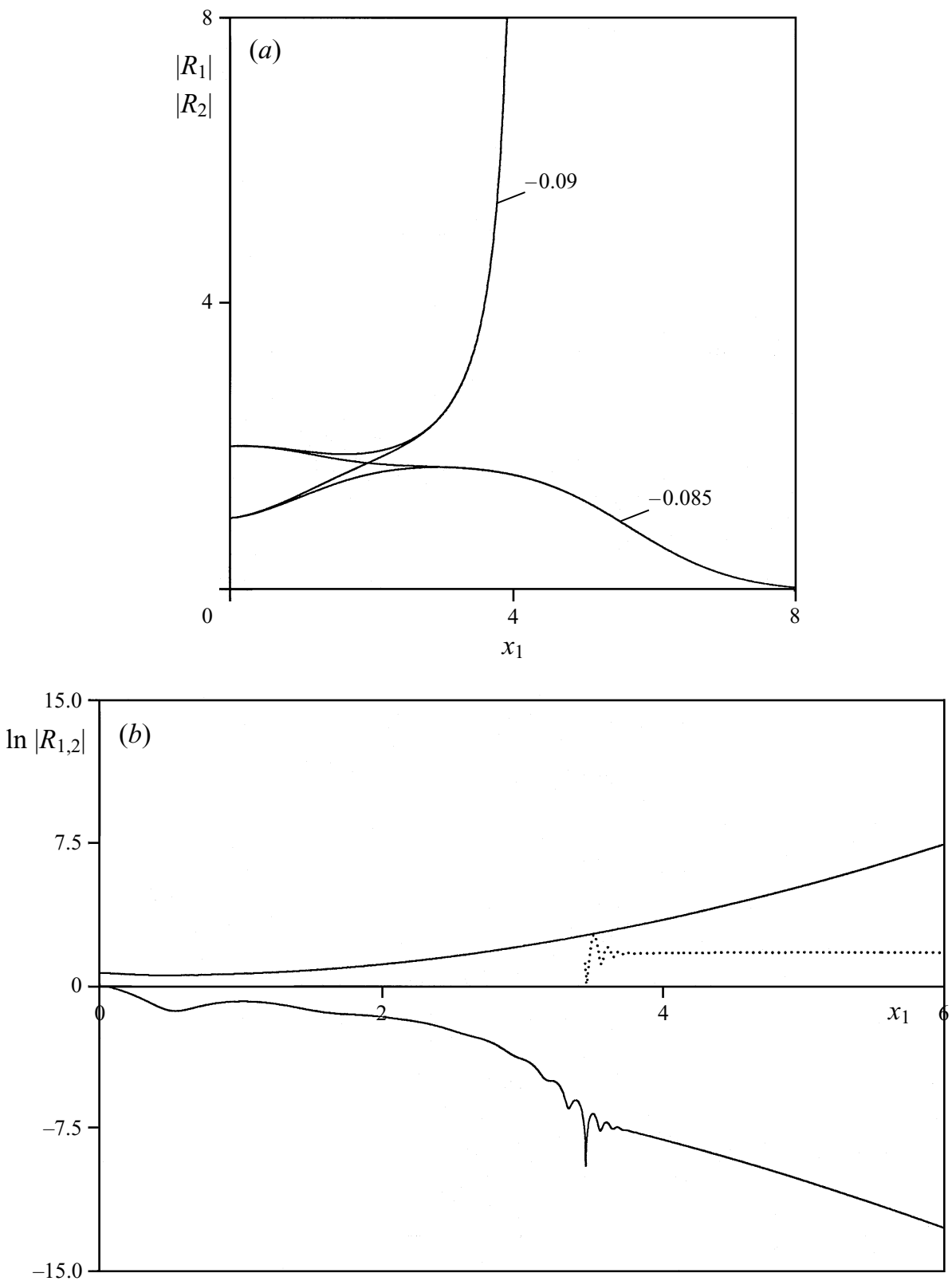

FIGURE $3(a, b)$. For caption see facing page.

where $\varepsilon=R^{-1 / 16}$ is the new small parameter. The expansions for the core flow are of the form

$$
\begin{gathered}
u=U_{0}(\bar{y})+\varepsilon^{4} x_{1} U_{1}(\bar{y})+\ldots+\varepsilon^{7}\left\{E\left[u^{(0)}+\varepsilon^{4} u^{(1)}+\ldots\right]+\text { c.c. }\right\}+\ldots, \\
v=\varepsilon^{8} V_{0}(\bar{y})+\ldots+\varepsilon^{7}\left\{E\left[v^{(0)}+\varepsilon^{4} v^{(1)}+\ldots\right]+\text { c.c. }\right\}+\ldots, \\
w=\varepsilon^{9}\left\{E\left[w^{(0)}+\varepsilon^{4} w^{(1)} \ldots\right]+\text { c.c. }\right\}
\end{gathered}
$$



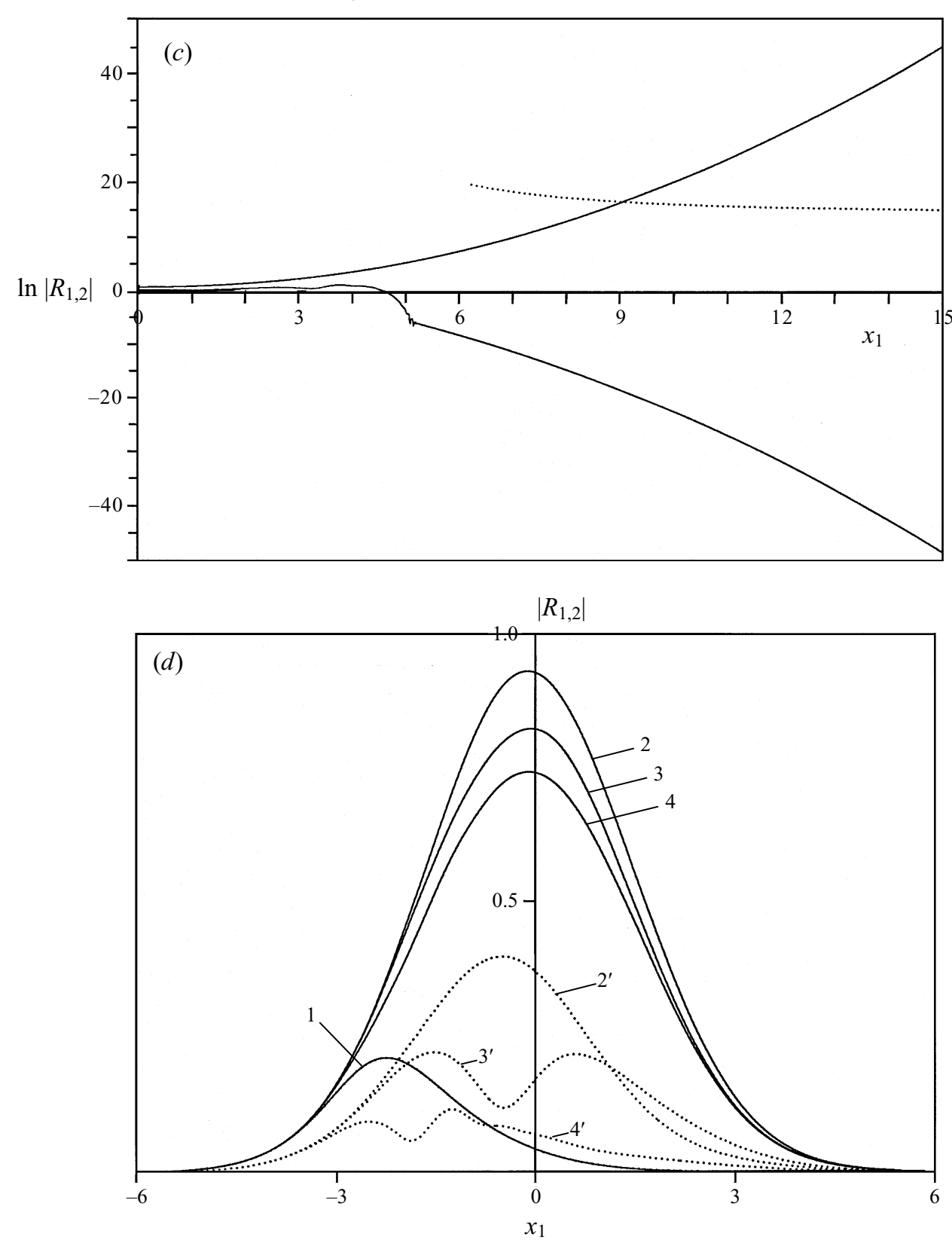

FIGURE 3. VWI with unequal input disturbances from the numerical solution of (3.41), (3.42), (3.55). (a) The amplitudes vs. $x_{1}, a=-1+0.5 \mathrm{i}, c=-0.5, d=0, R_{10}=1, R_{20}=2$; the decaying solution is for $\tilde{e}_{0}=\tilde{e}_{1}=-0.085$; the singular solution is for $\tilde{e}_{0}=\tilde{e}_{1}=-0.09$. (b) The logarithm of the amplitudes vs. $x_{1}, a=-1+0.5 \mathrm{i}, c=0.5, d=0, R_{10}=1, R_{20}=2, \tilde{e}_{0}=1, \tilde{e}_{1}=0 ; \cdots$, the function $20\left|R_{1} R_{2}^{*}\right| x_{1}^{3 / 2}$ shown for comparison with (3.57). (c) As in (b) but with $\tilde{e}_{0}=\tilde{e}_{1}=0.01 ; \cdots$, the function $\frac{1}{3}\left|R_{1} R_{2}^{*}\right| x_{1}^{5 / 2}$ shown for comparison with (3.58). (d) Solution of (3.41), (3.42), (3.60) with $a=-1+0.5 \mathrm{i}, c=-0.5, d=0$; curve 1 , equal waves, $R_{10}=R_{20}=1, e_{0}=e_{1}=10$; the other plots are for nonsymmetric input with $R_{10}=1, R_{20}=0.5$; curves $2,2^{\prime}, e_{0}=e_{1}=0.1$; curves $3,3^{\prime}, e_{0}=e_{1}=1$; curves $4,4^{\prime}, e_{0}=e_{1}=10$. 


$$
p=\ldots+\varepsilon^{7}\left\{E\left[p^{(0)}+\varepsilon^{4} p^{(1)}+\ldots\right]+\text { c.c. }\right\}+\ldots,
$$

where $E=\exp \left[\mathrm{i}\left(\alpha_{0} X-\Omega T\right)\right]$ with real $\alpha_{0}, \Omega$.

The leading-order wave terms $u^{(0)}, v^{(0)}, w^{(0)}, p^{(0)}$ are again given by (3.6)-(3.9), the main difference being that now we are not assuming a simple-harmonic $Z$-dependence in the flow, see below. The normal velocity correction $v^{(1)}$ is governed by

$$
\begin{gathered}
\left(U_{0}-c_{0}\right)\left(\frac{\partial^{2} v^{(1)}}{\partial \bar{y}^{2}}-\alpha_{0}^{2} v^{(1)}\right)-U_{0}^{\prime \prime} v^{(1)}=S_{1} \frac{\partial r}{\partial x_{1}}+\mathrm{i} x_{1} r S_{2}+\mathrm{i} S_{3} \frac{\partial^{2} r}{\partial Z^{2}}, \\
v^{(1)}(\bar{y}=0)=\mathrm{i} \alpha_{0} b_{1}^{-1} \varphi^{\prime}(0)\left(-\mathrm{i} \alpha_{0} c_{0}\right)^{-1 / 2} r, \quad v^{(1)}(\bar{y} \rightarrow \infty) \rightarrow 0,
\end{gathered}
$$

with $S_{1}, S_{2}, S_{3}$ given by (3.11), (3.12). Hence near the critical level as $\bar{y} \rightarrow \bar{y}_{c}$ the formula (3.16) holds, with $v^{(6)}$ replaced by $v^{(1)}$ and the slow-time dependence omitted for simplicity, the other significant change being also that the jump condition is now written as

$$
Q_{2}^{+}-Q_{2}^{-}=\Delta_{1} \frac{\partial r}{\partial x_{1}}+\mathrm{i} \Delta_{3} \frac{\partial^{2} r}{\partial Z^{2}}+r\left\{\mathrm{i} x_{1} \Delta_{2}+\mathrm{i} \alpha_{0} b_{1}^{-1}\left[\varphi^{\prime}(0)\right]^{2}\left(-\mathrm{i} \alpha_{0} c_{0}\right)^{-1 / 2}\right\} .
$$

In the buffer zone we take $y_{1}=\left(\bar{y}-\bar{y}_{c}\right) \varepsilon^{-2}$ to be of $O(1)$. The flow functions expand in the form

$$
\begin{gathered}
u=c_{0}+\varepsilon^{2} b_{1} y_{1}+\varepsilon^{4} d_{0} x_{1}+\varepsilon^{6}\left(\frac{1}{6} b_{3} y_{1}^{3}+d_{1} x_{1} y_{1}\right) \\
+\varepsilon^{8} \tilde{U}_{0}+\ldots+\varepsilon^{7}\left\{E\left[\tilde{u}_{0}+\varepsilon^{2} \tilde{u}_{1}+\varepsilon^{4} \ln \varepsilon \tilde{u}_{2,0}+\varepsilon^{4} \tilde{u}_{2}+\ldots\right]+\text { c.c. }\right\}+\ldots, \\
v=\varepsilon^{8} V_{0}\left(\bar{y}_{c}\right)+\varepsilon^{10} y_{1} V_{0}^{\prime}\left(\bar{y}_{c}\right)+\varepsilon^{12} \tilde{V}_{0}+\ldots \\
+\varepsilon^{7}\left\{E\left[\tilde{v}_{0}+\varepsilon^{2} \tilde{v}_{1}+\varepsilon^{4} \tilde{v}_{2}+\varepsilon^{6} \ln \varepsilon \tilde{v}_{3,0}+\varepsilon^{6} \tilde{v}_{3}+\ldots\right]+\text { c.c. }\right\}+\ldots, \\
w=\varepsilon^{8} \tilde{W}_{0}+\ldots+\varepsilon^{7}\left\{E\left[\tilde{w}_{0}+\ldots\right]+\text { c.c. }\right\}+\ldots, \\
p=\ldots+\varepsilon^{7}\left\{E\left[\tilde{p}_{0}+\ldots .+ \text { c.c. }\right\}+\ldots\right.
\end{gathered}
$$

The wave and the vortex dynamics in the buffer is also similar to the flow regime in $\S 3$. In particular, the main wave terms $\tilde{v}_{0}, \tilde{w}_{0}, \tilde{p}_{0}$ are given by (3.24). The wave component $\tilde{v}_{3}$ in (4.10) is coupled with the induced vortex by the relation of the form (3.25). Next, the vortex velocities, i.e. $\tilde{W}_{0}$ in (4.11) and the wave-induced part of $\tilde{U}_{0}, \tilde{V}_{0}$ in (4.9), (4.10), satisfy (3.28), (3.29) but with the jump value (3.33) omitted due to the increased spanwise lengthscale. It can also be verified that the viscous critical layer performs the same functions as in $\$ 3$. Hence, to obtain the governing formulation for the flow at hand we equate the jump in (4.8) with the corresponding jump supported by the inner layers (as calculated in $\S 3$ ) but with $J_{1} \equiv 0$. All this summarizes in the following equation:

$$
\begin{aligned}
\left(\frac{\pi c_{0} b_{3}}{b_{1}^{3}} \operatorname{sgn}\left(\alpha_{0} b_{0}\right)\right. & \left.+\mathrm{i} \Delta_{1}\right) \frac{\partial r}{\partial x_{1}}-\Delta_{3} \frac{\partial^{2} r}{\partial Z^{2}}+r\left\{-\frac{\alpha_{0}}{b_{1}}\left[\varphi^{\prime}(0)\right]^{2}\left(-\mathrm{i} \alpha_{0} c_{0}\right)^{1 / 2}\right. \\
& \left.-x_{1}\left[\Delta_{2}+\frac{\mathrm{i} \pi \alpha_{0}}{b_{1}^{2}}\left(d_{2}-\frac{b_{3} d_{0}}{b_{1}}\right) \operatorname{sgn}\left(\alpha_{0} b_{1}\right)\right]\right\} \\
& +\frac{\alpha_{0} \pi^{3 / 2}}{2 b_{1}^{3} c_{0}^{1 / 2}} r \int_{-\infty}^{x_{1}} \frac{\partial}{\partial Z}\left(r(s, Z) \frac{\partial r^{*}(s, Z)}{\partial Z}\right) \frac{\mathrm{d} s}{\left(x_{1}-s\right)^{1 / 2}}=0,
\end{aligned}
$$

for the amplitude of the wave pressure $r\left(x_{1}, Z\right)$. 


\subsection{Properties of the amplitude equation}

To simplify the notation, the amplitude equation is now written in the following general form:

$$
a \frac{\partial r}{\partial x}+b \frac{\partial^{2} r}{\partial z^{2}}+(c x+d) r+e_{0} r \int_{x_{0}}^{x} \frac{\partial}{\partial z}\left(r \frac{\partial r^{*}}{\partial z}\right) \frac{\mathrm{d} s}{(x-s)^{1 / 2}}=0
$$

where the constant coefficients $a, c, d$ are complex but $b, e_{0}$ are real; the specific expressions for the coefficients in terms of the basic-state flow field are easily derivable from (4.13). The equation obtained represents a generalization of the earlier Wu's (1993) limiting form of the non-equilibrium critical-layer flow in that a non-parallel effect is explicitly present at the leading order. Also Wu et al. (1996) show that (4.14) with $c=0$ and the coefficients $a, d$ purely imaginary applies to nonlinear wave packets in the upper-branch regime of the Tollmien-Schlichting instability for a flat-plate boundary layer. Until now solutions of (4.14) were only analysed for the special case of the last flow. Here we adopt a more general approach and treat the coefficients in (4.14) as arbitrary complex values (but see below in \$4.2.2). The wave pressure $r(x, z)$ is then supposed to be specified at $x=x_{0}$, with $x_{0}$ either finite or negative infinite depending on the context, so that (4.14) can be solved for example numerically marching downstream with suitable boundary conditions in the spanwise direction.

\subsubsection{Secondary instabilities and correctness of the initial-value problem}

Suppose that $r=r_{0}$ is a constant locally in the vicinity of a certain point $x=X_{0}$ where the flow is perturbed by a short-scale disturbance of the form

$$
r=r_{0}+\left[A_{1} \exp (\lambda x+\mathrm{i} \beta z)+A_{2} \exp \left(\lambda^{*} x-\mathrm{i} \beta z\right)\right]+\ldots,
$$

with $A_{1,2}$ being small constant (complex) amplitude factors. Substitution into (4.14) shows that the growth rate $\lambda$ satisfies a cubic equation with two roots given by

$$
\lambda_{1,2}=\beta^{2} b|a|^{-2}\left(a_{r} \pm \mathrm{i} a_{i}\right)+\ldots \text { as } \beta \rightarrow \infty .
$$

These are controlled by the linear operator $a \partial r / \partial x+b \partial^{2} r / \partial z^{2}$ and result in Hadamard's ill-posedness of the initial-value problem in the case $a_{r} b>0$ or, by contrast, in fast decay of short waves if $a_{r} b<0$. In this paper we are not concerned with ill-posed formulations; the case $a_{r} b<0$ is assumed subsequently. Even then short-scale perturbations can still be unstable due to the third root $\lambda_{3}$ with the limit property

$$
\lambda_{3}=\pi\left(e_{0} / b\right)^{2}\left|r_{0}\right|^{4}+\ldots \text { as } \beta \rightarrow \infty \text {. }
$$

This is from a balance between the integral term and the second $z$-derivative in the equation, and an equivalent conclusion was drawn in $\mathrm{Wu}$ et al. (1996). The typical behaviour of the third root is illustrated in figure 4.

The short-wave instability (4.17) has no immediate impact on the correctness of the formulation, nevertheless the disturbance growth can be sufficiently strong to affect finite-difference computations especially on coarser grids. It can also be shown that the instability vanishes if the entire solution including disturbances is real. Partly because of that the properties of complex-valued and purely real amplitude distributions considered below prove to be significantly different. The final feature to be mentioned here is the importance of the spanwise diffusion term $b \partial^{2} r / \partial z^{2}$ in the wave equation. If $b=0$ then the initial-value problem is ill-posed because unstable modes with unbounded growth rates $\lambda$ of $O\left(\beta^{4 / 3}\right)$ can be found for large $\beta$, again due to the convolution integral. 


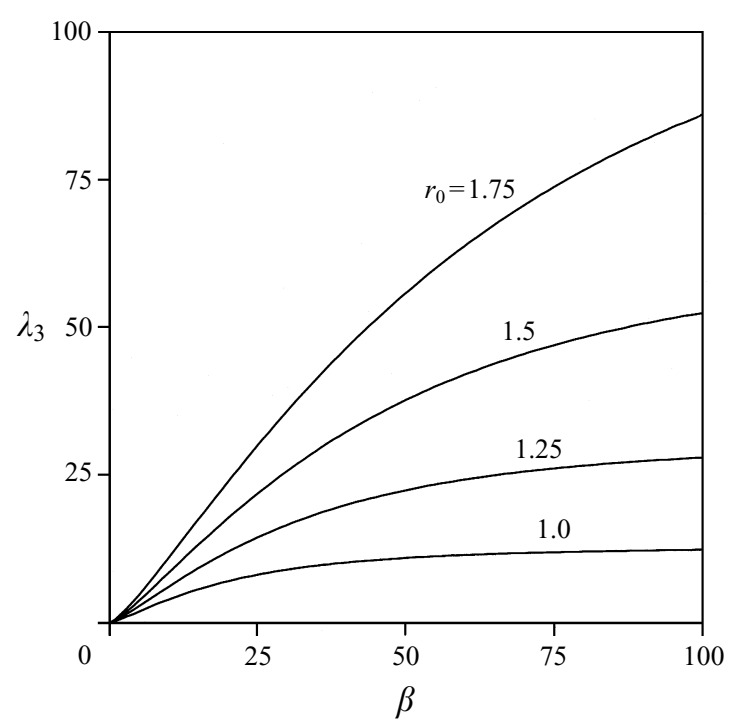

Figure 4. Short-scale instability in VWI governed by (4.14). Third root $\lambda_{3}$ vs. $\beta$. The parameters $b=0.05, a_{r}=-1, a_{i}=0.5, c_{0}=1, e_{0}=0.1$ and the values of $r_{0}$ as shown are typical for the flow regimes considered in $\S 4$.

A similar approach applies to the analysis of side-band secondary instability of a single oblique-wave disturbance; see e.g. Benjamin \& Feir (1967). Owing to the special form of the nonlinearity the linear wave, $r_{0}(x, z)=r_{00} \exp \left[\mathrm{i} \beta z-c x^{2} /(2 a)+\right.$ $\left.\left(b \beta^{2}-d\right) x / a\right]$, represents an exact solution for any constant $r_{00}$ and any real $\beta$, as in $\mathrm{Wu}$ (1993), Wu et al. (1996). Then, provided that $r_{0}$ is growing exponentially downstream, small-amplitude side modes of the form

$$
r=r_{0}(x, z)+\varepsilon\left[r^{+}(x) \exp [\mathrm{i}(\beta+\delta) z]+r^{-}(x) \exp [\mathrm{i}(\beta-\delta) z]\right]+O\left(\varepsilon^{2}\right),
$$

with $\varepsilon \ll 1$ and the wavenumber perturbation $\delta$ of $O(1)$, exhibit super-exponential growth

$$
r^{+}(x)=\frac{A^{+}}{r_{0}^{*}} \exp [Q(x)], \quad r^{-}(x)=\frac{A^{-}}{r_{0}^{*}} \exp \left[Q^{*}(x)\right],
$$

with $A^{ \pm}$constant and $Q(x)$ given by

$$
\frac{\mathrm{d} Q}{\mathrm{~d} x}=\left(\delta e_{0} \pi^{1 / 2}\right)^{2 / 3}\left|r_{0}\right|^{4 / 3}\left[\mathrm{i} \beta \frac{a_{i}}{|a|^{2}} \pm \frac{1}{|a|}\left(\delta^{2}-\beta^{2} a_{i}^{2}|a|^{-2}\right)^{1 / 2}\right]+\ldots,
$$

at the main order for large $x$. The growth requirement $\operatorname{Real}(\mathrm{d} Q / \mathrm{d} x)>0$ specifies a range of $\delta$ for instability. As with the short-wave instability given by (4.17), the fast growth of side bands in (4.20) holds also for the flow considered in Wu et al. (1996). It can be verified however that the side-band instability mechanism is not operational on real (including disturbances) solutions, in accord with the quasi-parallel property above.

\subsubsection{High-amplitude regimes}

On replacing $r(x, z)$ by an expression of the form $r(x, z) \exp \left[i\left(\gamma_{1} x^{2}+\gamma_{2} x\right)\right]$ with $\gamma_{1}=-c_{i}\left(2 a_{r}\right)^{-1}, \gamma_{2}=-d_{i} a_{r}^{-1}$, the controlling equation (4.14) remains unaltered except that the coefficients $c, d$ become real. We shall also assume that $b$ is positive, then $a_{r}$ 
must be negative for correctness, and we may take $a_{r}=-1$ without loss of generality (the case $a_{r}=0$ when the above transformation becomes singular is studied in Wu et al. 1996).

Consider first the flow initiated at a finite station within the interaction domain by disturbances of increased amplitude of order $\varepsilon_{1}^{-1} \gg 1$, say. The balance between the nonlinear term and the spatial derivatives in (4.14) requires then $\Delta x=O\left(\varepsilon_{1}^{4}\right)$, $\Delta z=O\left(\varepsilon_{1}^{2}\right)$, so that after a suitable scaling transformation the linear growth/decay terms vanish, $c=d=0$ in effect. In computations for this and subsequent regimes our main interest was in spanwise-periodic flows, partly for simplicity but also in order to compare the flow properties with shorter-scale solutions in \$3, Smith et al. (1993) and elsewhere. The numerical work was based on two different schemes, iterative and predictor-corrector, applied to the form (4.14) or, equivalently, to the coupled real equations for the magnitude $\rho(x, z)$ and phase $\varphi(x, z)$ of the wave pressure:

$$
\begin{gathered}
a_{r} \frac{\partial \rho}{\partial x}+b \frac{\partial^{2} \rho}{\partial z^{2}}+\rho\left[-a_{i} \frac{\partial \varphi}{\partial x}-b\left(\frac{\partial \varphi}{\partial z}\right)^{2}+c_{r} x+d_{r}\right. \\
\left.+e_{0} \int_{x_{0}}^{x} \frac{\partial}{\partial z}\left(\rho \frac{\partial \rho}{\partial z}\right) \frac{\mathrm{d} s}{(x-s)^{1 / 2}}\right]=0 \\
a_{r} \frac{\partial \varphi}{\partial x}+b \frac{\partial \varphi}{\partial z^{2}}+\frac{2 b}{\rho} \frac{\partial \rho}{\partial z} \frac{\partial \varphi}{\partial z}+\frac{a_{i}}{\rho} \frac{\partial \rho}{\partial x}+c_{i} x+d_{i}-e_{0} \int_{x_{0}}^{x} \frac{\partial}{\partial z}\left(\rho^{2} \frac{\partial \varphi}{\partial z}\right) \frac{\mathrm{d} s}{(x-s)^{1 / 2}}=0
\end{gathered}
$$

as follows from (4.14) on writing $r=\rho \exp (\mathrm{i} \varphi)$. Various simplifying circumstances (e.g. flow symmetry) are used to reduce the computational domain as reflected in illustrations.

The ultimate development of the present strong waves depends on the sign of the nonlinear coefficient $e_{0}$ and also, especially when $e_{0}$ is positive, on whether the amplitude $r$ is real or complex, see figure $5(a-c)$. These solutions have the same starting condition $r(x=0, z)=1+\frac{1}{2} \cos (2 \pi z)$ and a fixed value of $b$.

Negative values of $e_{0}$ have a destabilizing effect, the outcome being typically a finite-distance self-focusing of the amplitude into isolated singularities periodically spaced along the span; figure 5(a). If $x_{s}, z_{s}$ denote the singularity location within a chosen period then a balance of the three differential terms in (4.14) yields the local solution structure

$$
r=\left(x_{s}-x\right)^{-(1+\mathrm{i} M) / 4} \bar{R}(\zeta)+\ldots \text { as } x \rightarrow x_{s}-, z \rightarrow z_{s},
$$

with $\zeta=\left(z-z_{s}\right)\left(x_{s}-x\right)^{-1 / 2}$ of $O(1)$ and real constant $M$ as in Wu et al. (1996). The amplitude and phase of the function $\bar{R}(\zeta)=\bar{\rho}(\zeta) \exp (\mathrm{i} \bar{\varphi}(\zeta))$ then satisfy the equations

$$
\begin{gathered}
b\left(\bar{\rho}^{\prime \prime}-\bar{\rho}\left(\bar{\varphi}^{\prime}\right)^{2}\right)+\frac{1}{4}\left(a_{r}-M a_{i}\right) \bar{\rho}+\frac{1}{2} \zeta\left(a_{r} \bar{\rho}^{\prime}-a_{i} \bar{\rho} \bar{\varphi}^{\prime}\right)+\frac{e_{0} \bar{\rho}}{|\zeta|} \int_{0}^{\zeta}\left(\bar{\rho}^{2}\right)^{\prime \prime} \frac{t \mathrm{~d} t}{\left(\zeta^{2}-t^{2}\right)^{1 / 2}}=0, \quad \text { (4.24) } \\
b\left(\bar{\rho} \bar{\varphi}^{\prime \prime}+2 \bar{\rho}^{\prime} \bar{\varphi}^{\prime}\right)+\frac{1}{4}\left(a_{i}+M a_{r}\right) \bar{\rho}+\frac{1}{2} \zeta\left(a_{i} \bar{\rho}^{\prime}+a_{r} \bar{\rho} \bar{\varphi}^{\prime}\right)-\frac{2 e_{0} \bar{\rho}}{|\zeta|} \int_{0}^{\zeta}\left(\bar{\rho}^{2} \bar{\varphi}^{\prime}\right)^{\prime} \frac{t \mathrm{~d} t}{\left(\zeta^{2}-t^{2}\right)^{1 / 2}}=0 .
\end{gathered}
$$

Matching with a predominantly regular solution upstream yields the condition

$$
\bar{R}(\zeta)=R_{ \pm}|\zeta|^{-(1+M \mathrm{i}) / 2}+\ldots \text { as } \zeta \rightarrow \pm \infty,
$$

at the edges of the local region, with constants $R_{ \pm}$, in line with a singularity

$$
r(x, z)=R_{ \pm}\left|z-z_{s}\right|^{-(1+M \mathrm{i}) / 2}+\ldots \text { as } z \rightarrow z_{s} \pm,
$$



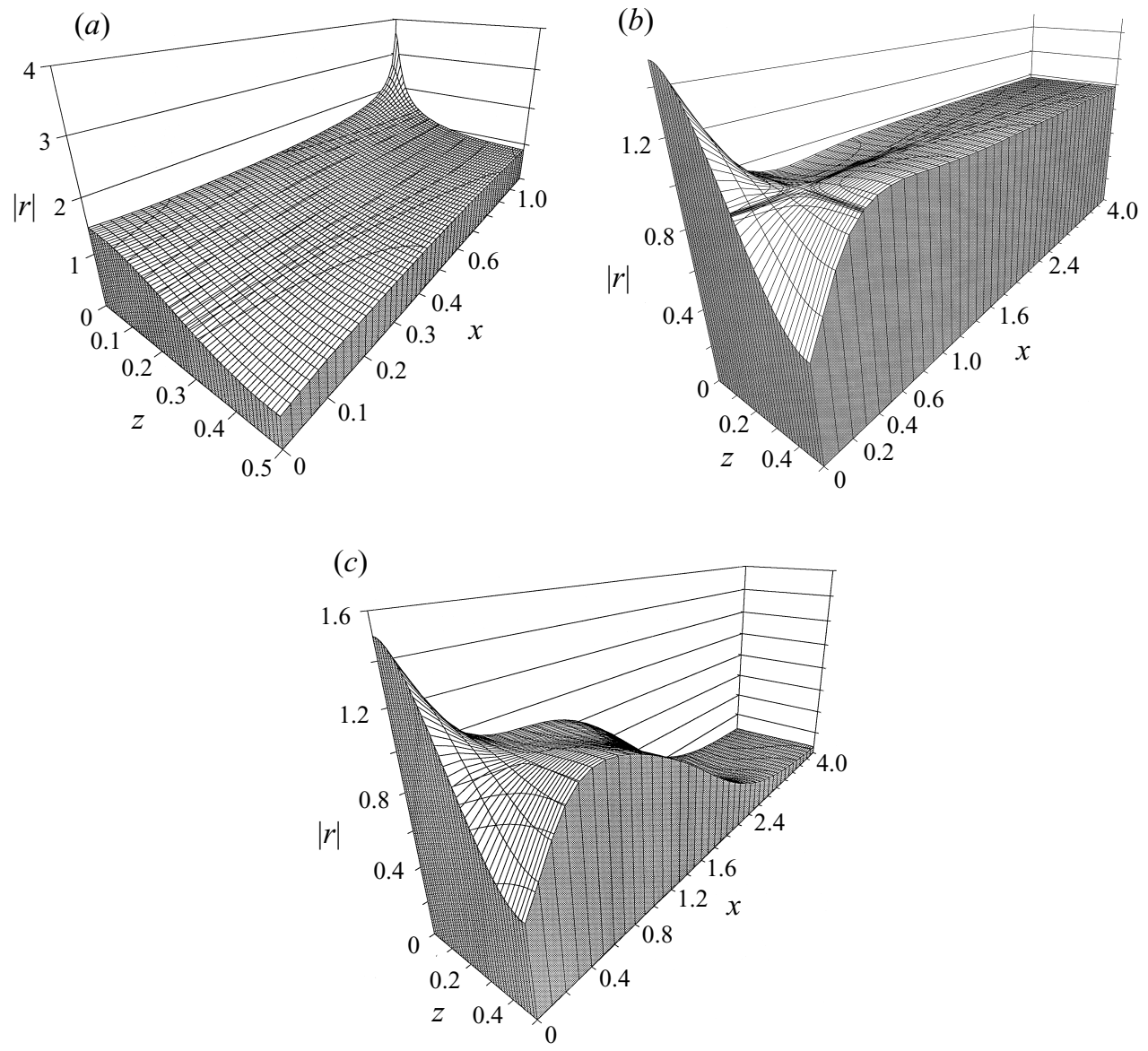

FIGURE 5. High-amplitude VWI; $|r|$ shown as a function of $x, z$, from computations for (4.14), $r(x=0)=1+0.5 \cos 2 \pi z$. (a) The blow-up singularity at $x_{s}=1.106$ in the flow with $e_{0}=-0.04$, $a=-1+0.5 \mathrm{i}, b=0.08 ;(b)$ nonlinear stabilization of real solutions, $e_{0}=0.1, a=-1, b=0.08 ;(c)$ nonlinear stabilization in the complex-valued solution, $e_{0}=0.1, a=-1+0.5 \mathrm{i}, b=0.08$.

in the spanwise pressure distribution at $x_{s}$. A straightforward comparison with the suggested local structure is made in figure $6(a)$, where $|r|^{-2}$ plotted against $z$ close to $x_{s}=1.106$ demonstrates the appearance of finite slopes at the origin in the limit.

Solutions of (4.24) for the case of symmetry, $\bar{\rho}^{\prime}(0)=\bar{\varphi}^{\prime}(0)=0$, and with $\bar{\varphi} \equiv$ $0, M=a_{i}=0$, are shown in figure $6(b)$. In computations the function $\bar{\rho}$ was specified by a Taylor series in a small interval $0<\zeta<\zeta_{0}$ in order to prevent the appearance of algebraic eigensolutions at the origin. Also the parameters $\bar{\rho}(0)=b=1$ are taken without loss of generality. As shown in figure 6(b), the required solution satisfying (4.26) is obtained when $e_{0}=-0.64065$ approximately.

The case $a_{i} \neq 0$ can be treated in a similar manner except that the appropriate choice has to be made in the $\left(e_{0}, M\right)$-plane. In particular, if $a_{i}$ is small then the solution expands in the form

$$
\left(\bar{\rho}, \bar{\varphi}, M, e_{0}\right)=\left(\tilde{\rho}_{0}, 0,0, \tilde{e}_{0}\right)+a_{i}\left(\tilde{\rho}_{1}, \tilde{\varphi}_{1}, \tilde{M}_{1}, \tilde{e}_{1}\right)+O\left(a_{i}^{2}\right),
$$

where $\tilde{\rho}_{0}, \tilde{e}_{0}$ correspond to the solution above, whilst the correction $\tilde{\varphi}_{1}$ is governed by 

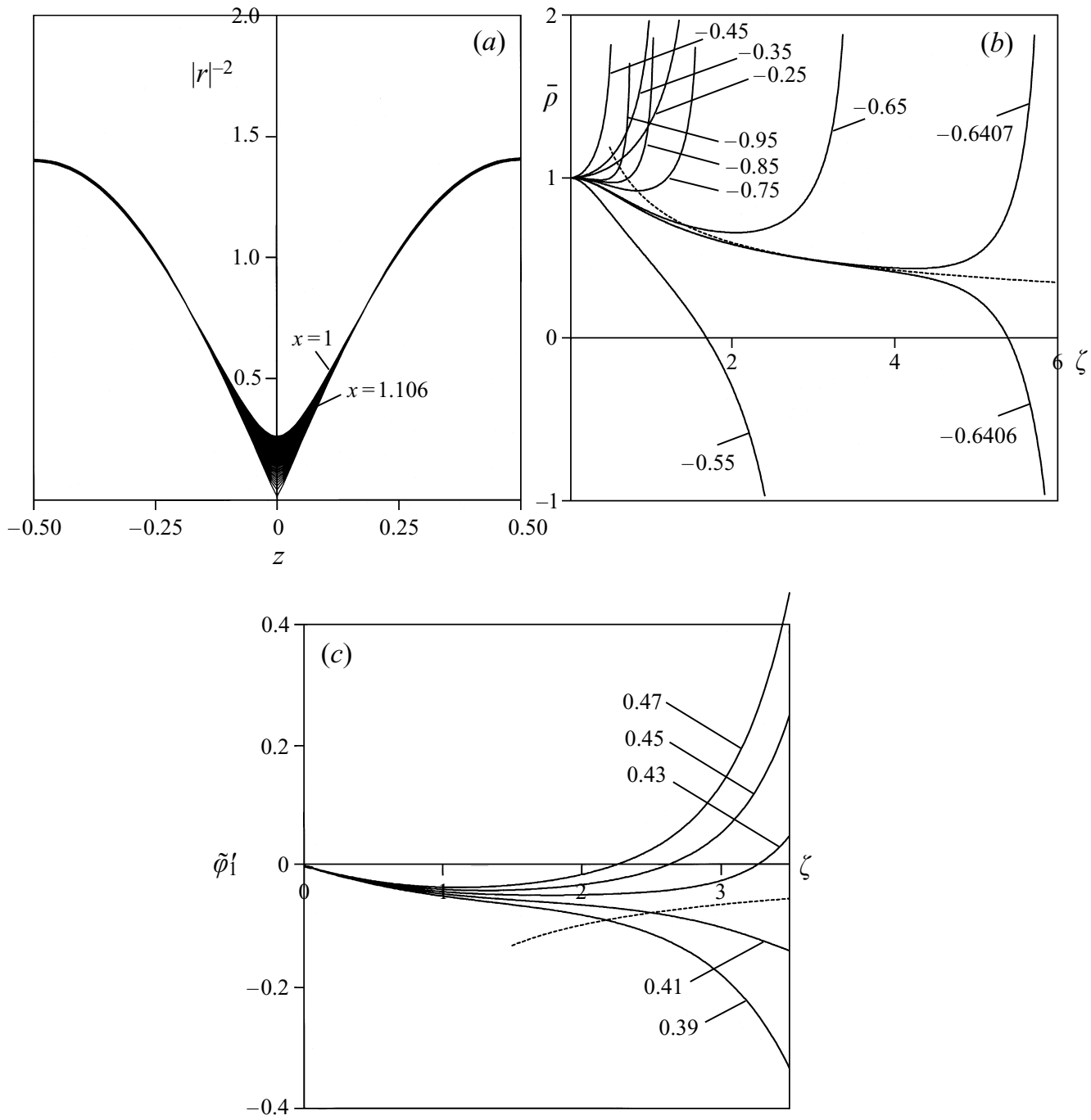

FiguRE 6. The break-up self-focusing. ( $a)|r|^{-2}$ vs. $z$ plotted from computations for figure $5(a)$ at the $x$-locations between 1 and 1.106 inclusive with step $\delta x=0.002 ;(b)$ Solutions of $(4.24), \bar{\rho}$ vs. $\zeta$, in the case $\bar{\varphi} \equiv 0, a_{i}=M=0, b=-a_{r}=1$ and varying $e_{0}$ marked on the plots (solid). Dashes represent the asymptote (4.26), $\bar{\rho}=0.84 \zeta^{-1 / 2}$, with the coefficient chosen to fit the neighbouring branches at $\zeta=3$. (c) $\tilde{\varphi}_{1}^{\prime}$ vs. $\zeta$ computed from (4.29) with $b=-a_{r}=1$ and varying $\tilde{M}_{1}$ (solid); the dashes show the asymptote $\tilde{\varphi}_{1}^{\prime}=-\tilde{M}_{1} /(2 \zeta)$ with $\tilde{M}_{1}=0.42$.

the linear forced equation

$$
\begin{aligned}
\frac{1}{2} a_{r} \zeta \tilde{\rho}_{0}^{2} \tilde{\varphi}_{1}^{\prime}+b\left(\tilde{\rho}_{0}^{2} \tilde{\varphi}^{\prime}\right)^{\prime}-\frac{2 \tilde{e}_{0} \tilde{\rho}_{0}^{2}}{\zeta} \int_{0}^{\zeta}\left(\tilde{\rho}_{0}^{2} \tilde{\varphi}_{1}^{\prime}\right)^{\prime} \frac{t \mathrm{~d} t}{\left(\zeta^{2}-t^{2}\right)^{1 / 2}} \\
=-\frac{1}{4}\left(1+\tilde{M}_{1} a_{r}\right) \tilde{\rho}_{0}^{2}-\frac{1}{4} \zeta\left(\tilde{\rho}_{0}^{2}\right)^{\prime} .
\end{aligned}
$$

As illustrated in figure 6(c), the behaviour of $\tilde{\varphi}_{1}^{\prime}$ as $\zeta \rightarrow \infty$ involves an exponentially growing term, $\tilde{\varphi}_{1}^{\prime}=O\left(\exp \left(-a_{r} \zeta^{2} / 4 b\right)\right)$ typically, except when $\tilde{M}_{1}(\approx 0.42)$ is chosen such that the coefficient of the exponential becomes zero and then $\tilde{\varphi}^{\prime}=-\tilde{M}_{1} /(2 \zeta)+\ldots$, 
as $\zeta \rightarrow \infty$, in accord with (4.26). Thus the solvability of the local formulation establishes a link between the parameters $a_{i}, M$.

Positive values of the nonlinear constant $e_{0}$ suggest nonlinear wave stabilization. The purely real solution in figure $5(b)$ has a constant limit $r \rightarrow r_{\infty}$ as $x \rightarrow \infty$. Because of the mode interactions the limit value $r_{\infty}$ is somewhat lower than the spanwise average $\langle r\rangle=1$ at the start of interaction; computations on various grids give $r_{\infty}=0.9762-0.9770$ approximately; see also figure $7(a)$. Further properties of the large- $x$ behaviour are obtained using an estimate for the convolution integral in the form

$$
I(x, z)=\int^{x} \frac{\partial}{\partial z}\left(r \frac{\partial r^{*}}{\partial z}\right) \frac{\mathrm{d} s}{(x-s)^{1 / 2}}=x^{-1 / 2} G_{0}(z)+x^{-3 / 2} G_{1}(z)+\ldots \text { as } x \rightarrow \infty .
$$

Then the amplitude equation simplifies to

$$
a \frac{\partial r}{\partial x}+b \frac{\partial^{2} r}{\partial z^{2}}+e_{0} x^{-1 / 2} r\left[G_{0}(z)+x^{-1} G_{1}(z)+\ldots\right]=0 .
$$

It can be verified (see figure $7 a$ ) that in the current regime the leading term in (4.30) vanishes, hence

$$
r=r_{\infty}+x^{-3 / 2} K_{1}(z)+\ldots \text { as } x \rightarrow \infty,
$$

where the first three-dimensional correction satisfies the equation

$$
b K_{1}^{\prime \prime}+e_{0} r_{\infty} G_{1}(z)=0
$$

with appropriate periodicity conditions. The zero main term in the estimate (4.30) implies that the interaction remains active even in the far field where, according to (4.31), the nonlinear contribution is small formally.

The nonlinear stabilization in the previous example appears in a certain reduction of the average amplitude, whereas the algebraic decay of the Fourier harmonics in (4.32) tends to be even slower than the exponential decay of linear modes. In the complex-valued solution in figure 5(c) however the mode interactions are far more active, suppressing the entire wave including the mean term soon after initiation of the flow. Analysis (omitted here) of the phase/amplitude equations (4.21), (4.22) indicates that a constant-limit solution is unstable to phase variations at large $x$ and therefore cannot be achieved unless $\varphi \equiv 0$, as in the previous case. A 'stable' alternative is hence $r \rightarrow 0$, as $x \rightarrow \infty$, leading again to the limiting equation (4.31), this time with a non-trivial main term $G_{0}(z)$. That, in turn, suggests the behaviour

$$
r=\frac{K_{0}(z)}{x^{\alpha}}+\frac{K_{1}(z)}{x^{\alpha+1 / 2}}+\frac{K_{2}(z)}{x^{\alpha+1}}+\ldots \text { as } x \rightarrow \infty,
$$

for the wave pressure, with the exponent $\alpha$ to be determined. It then proves that, with the boundary conditions $K_{j}^{\prime}(0)=K_{j}^{\prime}\left(\frac{1}{2}\right)=0$ appropriate for the case in figure $5(c)$, $K_{0}$ is constant whereas

$$
b K_{1}^{\prime \prime}+e_{0} G_{0} K_{0}=0,-a \alpha K_{0}+b K_{2}^{\prime \prime}+e_{0} K_{1} G_{0}=0,
$$

serve to determine $K_{1}, K_{2}$; periodicity of $K_{2}$ yields then

$$
\alpha=\frac{2 e_{0}^{2}}{a b} \int_{0}^{1 / 2}\left(\int_{0}^{z} G_{0}(s) \mathrm{d} s\right)^{2} \mathrm{~d} z .
$$

The last relation can be generalized to other cases of the spanwise periodicity. 


$\begin{array}{lccc}x & \alpha=\left(\alpha_{r}, \alpha_{i}\right) & \varphi_{m} & \rho(x, z=0) \\ 5 & (1.0312,0.2790) & -0.9772 & 0.0423 \\ 6 & (0.9758,0.2573) & -1.0184 & 0.0349 \\ 7 & (0.9403,0.2436) & -1.0532 & 0.0298 \\ 7.2 & (0.9347,0.2415) & -1.0595 & 0.0290 \\ 7.4 & (0.9295,0.2395) & -1.0656 & 0.0282 \\ 7.6 & (0.9246,0.2376) & -1.0715 & 0.0275 \\ 7.8 & (0.9200,0.2759) & -1.0772 & 0.0268 \\ 8 & (0.9157,0.2343) & -1.0827 & 0.0262\end{array}$

TABLE 1. Comparisons of the asymptote (4.34), (4.36) with computations, together with $\varphi_{m}$ from (4.38)

Comparisons of the asymptote (4.34), (4.36) with computations are summarized in figures $7(b)$ and $7(c)$, in table 1 and below. In figure $7(b)$ we plot the real and imaginary parts of the integral $I$ in (4.30) multiplied by $x^{1 / 2}$ at various $x$ in a somewhat larger $x$-range than shown in figure $5(c)$. Taking the product $I x^{1 / 2}$ as an approximation for $G_{0}(z)$ the formula (4.36) was then used to compute $\alpha$; the results are presented in table 1 . In particular, we have $\alpha=0.9157+0.2342 \mathrm{i}$ at the largest available $x$ value of 8 , and this can be compared with the estimates following directly from the amplitude/phase distributions. Thus the plots on the right-hand side in figure $7(c)$ indicate that the product $\rho x^{0.9157}$ becomes mostly independent of $x$ and $z$ as $x$ increases. Also the ratio of the centreline amplitudes taken at $x=8$ and $x=7.8$ (see table 1) yields $\alpha_{r}=0.8943$, not far from the value given earlier. As for the phase function, the analysis above implies the behaviour

$$
\varphi=-\alpha_{i} \ln x+\arg K_{0}+O\left(x^{-1 / 2}\right) \text { as } x \rightarrow \infty .
$$

Using the numerical solution we compute the $z$-averaged quantity

$$
\varphi_{m}=2 \int_{0}^{1 / 2} \varphi \mathrm{d} z
$$

the results are also shown in table 1 . The estimate for $\alpha_{i}$ is then obtained as $-\mathrm{d} \varphi_{m} / \mathrm{d}(\ln x)$; at $x=8$ this yields $\alpha_{i}=0.2189$, cf. the value 0.2342 determined by the integral condition. Finally, in order to verify the square-root decay of the spanwise phase variations (4.37), in figure 7(c) we plot the function $\left(\varphi+\alpha_{i} \ln x-\arg K_{0}\right) x^{1 / 2}$ at large $x$ using $\alpha_{i}=0.2189$ and the value of $\arg K_{0}=-0.5958$ derived from table 1 . The comparison seems to be supportive.

The analysis indicates, then, a rather unusual property, namely that the power of $x$ in the limit expansion (4.34) can be quite arbitrary, depending in a complicated manner on the initial conditions and integral properties of the flow. This is due to the term with $x^{-1 / 2}$ in the linearized form (4.31); further discussion of this point is given in Appendix D. The important feature, however, is that the positive real part of $\alpha$ in our example has a clear connection with the complex-valuedness of the solution: in the case of real $r$ with $a=a_{r}<0, b>0$, the relation (4.36) results in $\alpha<0$, i.e. the amplitude would grow downstream contrary to the properties observed in computations; hence the leading term in (4.30) must be set to zero.

\subsubsection{The effects of main-flow non-parallelism}

We return now to the full equation (4.14) and consider here the influence of the main-flow non-parallelism accumulated in the term $c x r$. There are many interesting 

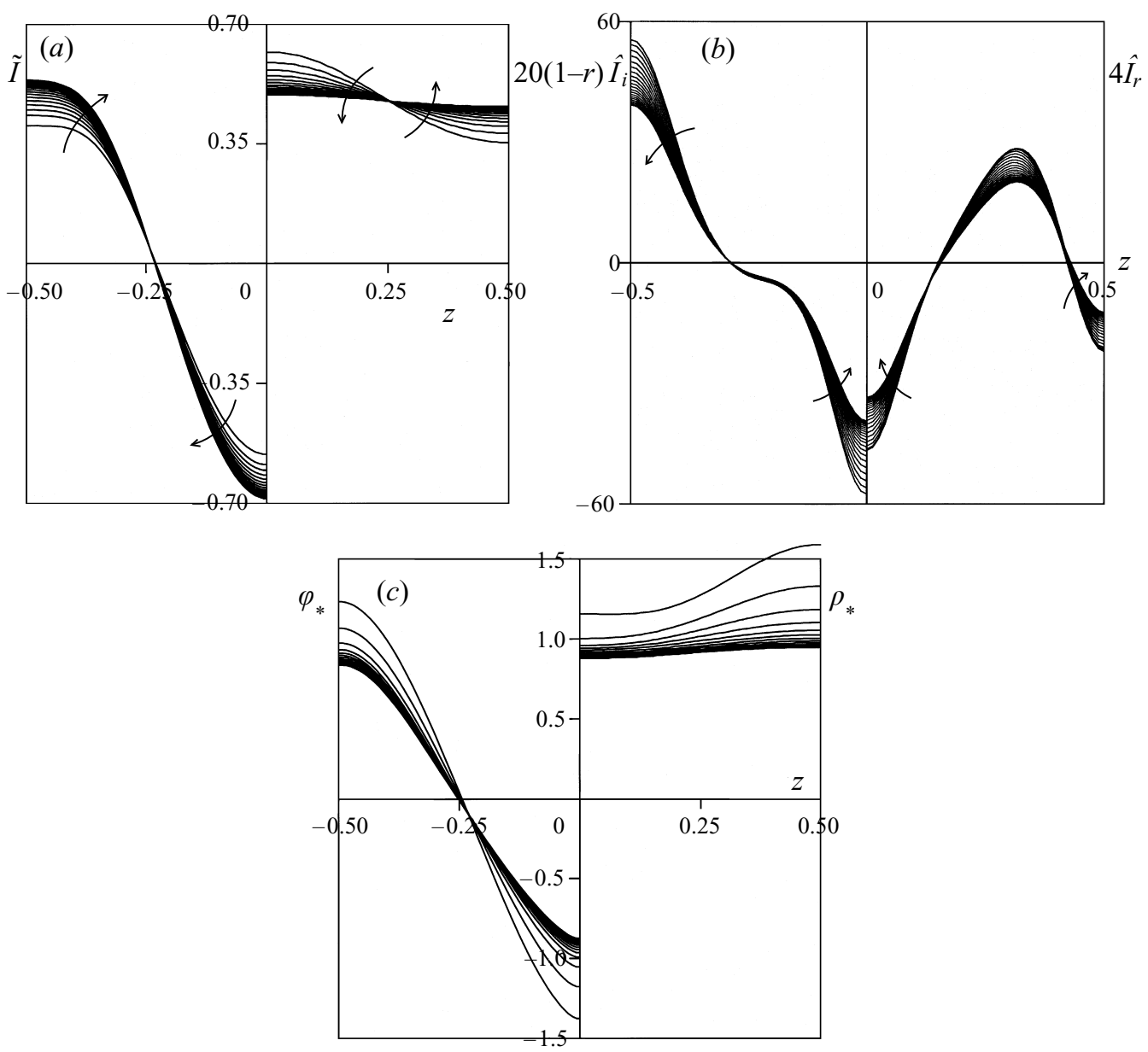

FIGURE 7. Nonlinearly stabilized high-amplitude VWI; arrows indicate increasing $x$. (a) Real solution; left, the function $\tilde{I}=x^{3 / 2} I(x, z)$ with $I$ as in (4.30) vs. $-z$, plotted for the values of $x$ from 2 to 8 inclusive with step $\delta x=0.4$ from the computation for figure $5(b)$; right, the function $20(1-r)$ vs. $z$ at the same $x$-stations as on the left. (b) The real (right) and the imaginary (left) parts of the function $\hat{I}=x^{1 / 2} I$ vs. $z$ (vs. $-z$ on the left) as $x$ increases from 2 to 4 with step $\Delta x=0.1$ in the solution in figure 5(c). (c) The spanwise phase variation $\varphi_{*}=\left(\varphi+\alpha_{i} \ln x-\arg K_{0}\right) x^{1 / 2}$ (left) and the normalized amplitude $\rho_{*}=5 \rho x^{0.9157}$ (right) vs. $z$ at the streamwise locations from $x=3.2$ to 8 plotted with the step $\delta x=0.2$ for the comparison with (4.37).

cases again that could be tackled; however, as in the previous subsection our intention here is to address a few basic ones. In the first we assume that $a_{r}<0, b>0$, and $c<0$. Within the linear regime this implies the non-parallel upper-branch behaviour

$$
r=\left[r_{\infty}^{(1)} \exp (\mathrm{i} \beta z)+r_{\infty}^{(2)} \exp (-\mathrm{i} \beta z)\right] \exp \left(-\frac{c}{2 a} x^{2}-\frac{d-b \beta^{2}}{a} x\right),
$$

for a pair of oblique modes with wavenumbers $\pm \beta$ and constant normalizing coefficients $r_{\infty}^{(1,2)}$. In the nonlinear computations below we use (4.39) as the starting condition as $x \rightarrow-\infty$; the theory is then relevant to the VWI induced by wave sources located far from the interaction region. 
In the solutions with $e_{0}<0$ illustrated in figure $8(a-d)$ a finite-distance breakdown occurs, the same as discussed earlier in conjunction with figure $5(a)$. The nonparallelism in the current setting suggests however a regular solution when $\left|e_{0}\right|$ is not sufficiently large; in particular $r$ is given by (4.39) when $\left|e_{0}\right| \ll 1$. This points to the presence of a marginal breakdown of the VWI at the threshold of the regular regimes as the nonlinearity increases, cf. $\S 3$ and Smith et al. (1993).

In the next series of computations $e_{0}$ is positive and the consequent nonlinear stabilization due to mode interactions adds to the linear decay downstream to produce an abrupt decrease in the wave amplitude, as illustrated in figures $9(a-j)$ for the case of equal incoming waves, $r_{\infty}^{(1)}=r_{\infty}^{(2)}$. In the region of the most rapid decay the real part of the solution contains several spikes which are especially pronounced at higher nonlinearity, see figures $9(h)$ and $9(j)$. A version of the same formulation but with unequal input waves reveals similar trends, as shown in figures $9(k)$ and $9(l)$.

The situation becomes far more complicated when the linear waves are unstable downstream owing to positive $c$ or, when $c$ is zero, to positive $d$ in (4.14). The initial amplitude then must be specified at a finite location $x_{0}$, and we can further confine ourselves to the case $e_{0}>0$, for otherwise the solution always seems to become singular at a finite distance according to our computations not presented here. For the chosen combination of properties the shorter-scale theories in Smith et al. (1993) and $\S 3$ would predict a flow development towards the Brown et al. (1993)-Hall \& Smith (1991) equilibrium saturation in certain cases, and so one of our aims here will be to examine the analogue of that saturation (and its likelihood) in the multi-mode regime.

In the case of real amplitudes first the wave development depends on whether a nontrivial mean component is present along the span, as illustrated in figure $10(a-e)$. When a non-zero mean is contained in the initial condition (figure 10a) the spanwise profile of the amplitude becomes virtually flat soon after the start of interaction; further downstream the wave system degenerates into a single planar disturbance. If however the pressure has zero average at the start then the VWI evolves towards saturation, as shown in figures $10(b)$ and $10(c)$. In the large- $x$ limit the nonlinear/nonparallel balance in (4.14) indicates that

$$
r=x^{1 / 4}\left(\frac{2 c}{\pi e_{0}}\left(z_{0}^{2}-z^{2}\right)\right)^{1 / 2}+\ldots \text { as } x \rightarrow \infty,
$$

in the case of symmetry at $z=0$, with $z_{0}=1 / 2$ in the example in figure $10(b)$. A comparison in figure $10(\mathrm{c})$ is favourable, and we can also add that the values of $x^{-1 / 4} r(x, z)=1.3313,1.3413,1.3549$ at the centreline $(z=0)$ obtained numerically at $x=8,10,15$ respectively seem to be in keeping with the limit value 1.4105 given by (4.40).

A similar saturation phenomenon is observed also in a strictly parallel flow, with $c=0$ and $d>0$, as shown in figure $10(d)$. The appropriate limiting form $r=$ $x^{-1 / 4} r^{0}(z)+\ldots$ for $x \rightarrow \infty$ yields the simple controlling equation

$$
\frac{\mathrm{d} z}{\mathrm{~d} q}= \pm\left(\frac{1+\beta q}{4 a q\left(q_{\max }-q\right)}\right)^{1 / 2}
$$

where $q=\left(r^{0}\right)^{2}, A=b d^{-1}, B=\pi e_{0} d^{-1}$ and $q_{\max }$ denotes the maximum value of $\left(r^{0}\right)^{2}$ over the period. The comparison is made in figure 10(e).

The corresponding regimes for a complex-valued amplitude are not so well understood yet in their limit properties and also raise interesting questions regarding better 


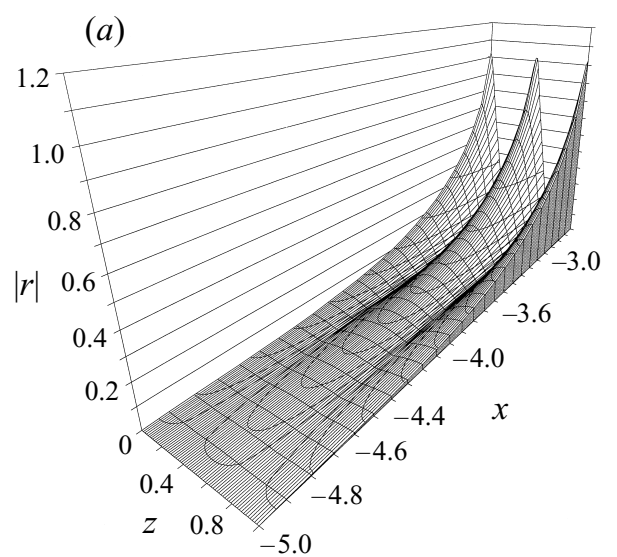

(b)

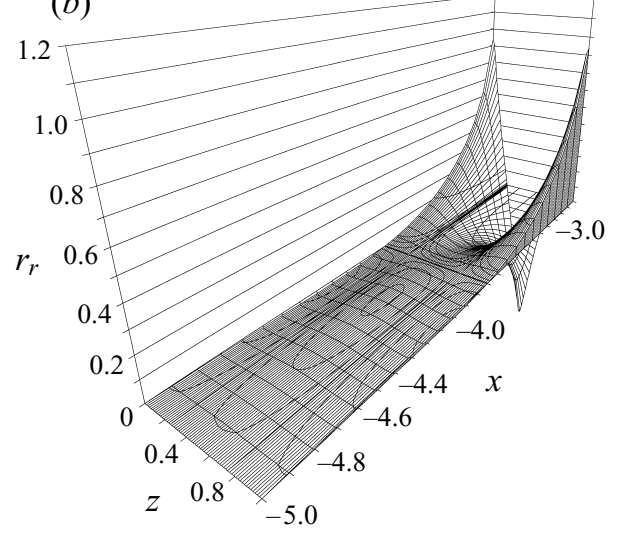

FIGURE $8(a, b)$. For caption see facing page.

organization of the computational process. An illustration is given in figure $11(a-c)$. When the spanwise average pressure is zero (see figure 11a, also figure 13c) the amplitude exhibits steep, predominantly linear, initial growth followed by an abrupt decrease similar to that in figures $9(g), 9(i)$ and $9(k)$. The subsequent development seems to be mostly smooth with the amplitude showing mild oscillations about the slow overall decay. (By comparison, the real-solution case saturates as in figure 10b). Estimates derived from the data for figure 11(a) and from larger- $x$ computations on coarser grids point to a decay rate $|r|=O\left(x^{-1 / 4}\right)$ or close to that. The wave behaviour in the limit as $x \rightarrow \infty$ involves an additional spanwise splitting in the amplitude distribution, we believe, and a promising candidate for the core of the limit structure is described in Appendix E. However the analysis lacks completeness and so a direct comparison with computations is inappropriate here. The wave development in the equally interesting case of a non-zero average tends to be even more complicated; see figures $11(b)$ and $11(c)$. In the corresponding real solution the three-dimensional components degenerate, leaving the planar component to evolve in a linear fashion. A similar scenario is most unlikely in the complex case, on the other hand, because of the powerful secondary instability mechanism discussed earlier in this section. The final comment we would make here concerns termination of the numerical solution 

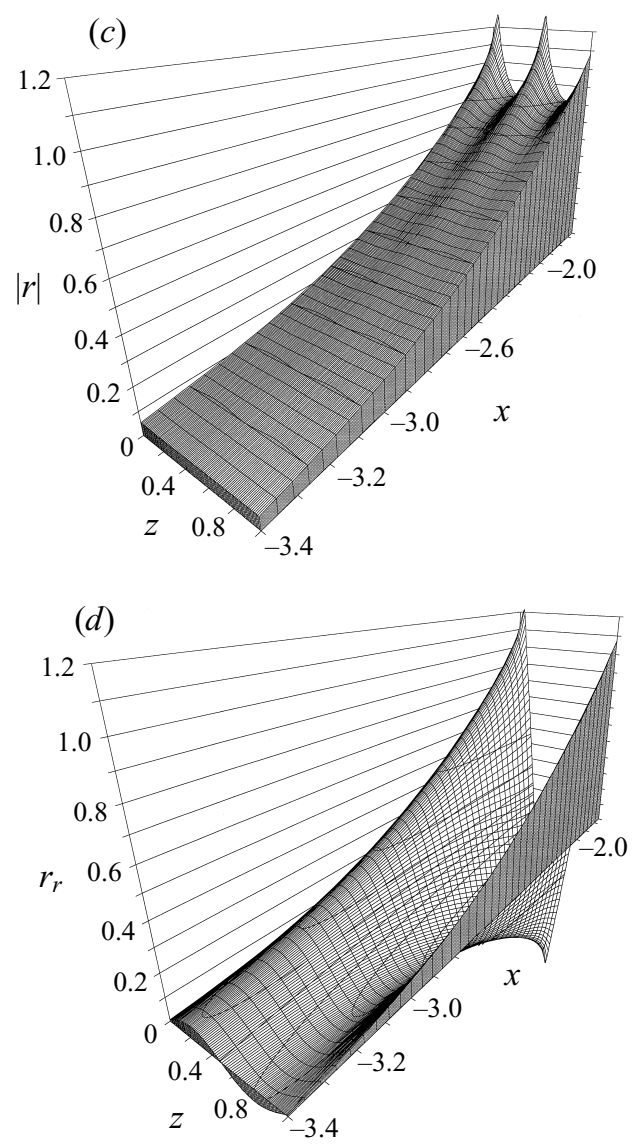

FIGURE 8. Finite-distance termination of the VWI induced far upstream; solution of (4.14), (4.39) with $a=-1+0.5 \mathrm{i}, b=0.02, c=-1, d=0, e_{0}=-0.075, \beta=2 \pi .(a, b)$ The magnitude $|r|$ and the real part $r_{r}$ for the case of equal waves, $r_{\infty}^{(1)}=r_{\infty}^{(2)}=1$; the singular station at $x_{s} \approx-2.6 .(c, d)|r|$ and $r_{r}$ for a strongly asymmetric input, $r_{\infty}^{(1)}=1, r_{\infty}^{(2)}=0.01$, singularity at $x_{s} \approx-1.65$. In both cases the computation commences at $x=-5$.

in the last example. In computations with a fixed $z$-grid but with $\Delta x$ decreasing from 0.01 to 0.0025 , in successive runs, the iterations repeatedly fail at or near the last $x$-section shown in the figures, $x \approx 12.01$. The terminal station varies with the $x$-step a little, but the trends in the solution seem to remain unaltered. The results suggest a possible singularity in the flow solution associated perhaps with the growing oscillations in the phase function that are discernible in figure $11(c)$; this is another intriguing case requiring further study.

\section{Discussion}

This work has examined theoretically certain nonlinear three-dimensional interactions between induced streamwise vortices and inflectional waves when the input waves upstream are only slightly three-dimensional. The present interactions can arise when a two-dimensional boundary layer with an inflectional streamwise velocity profile locally is in the presence of almost two-dimensional input waves. As in Smith et al. (1993) and related studies, the wave amplitudes provoking the current nonlinear 

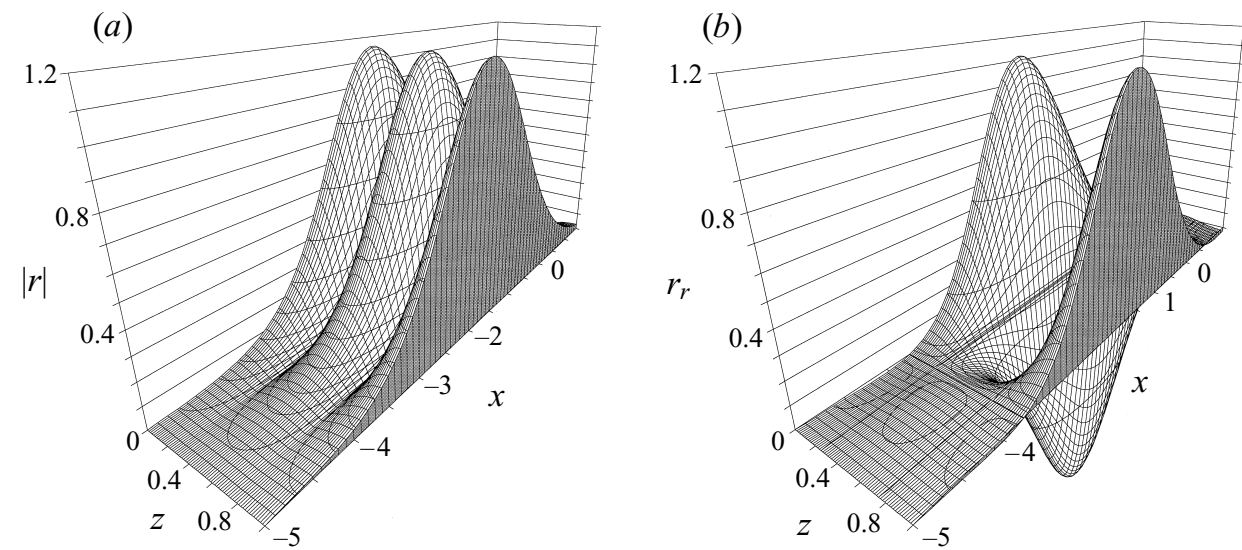

(c)
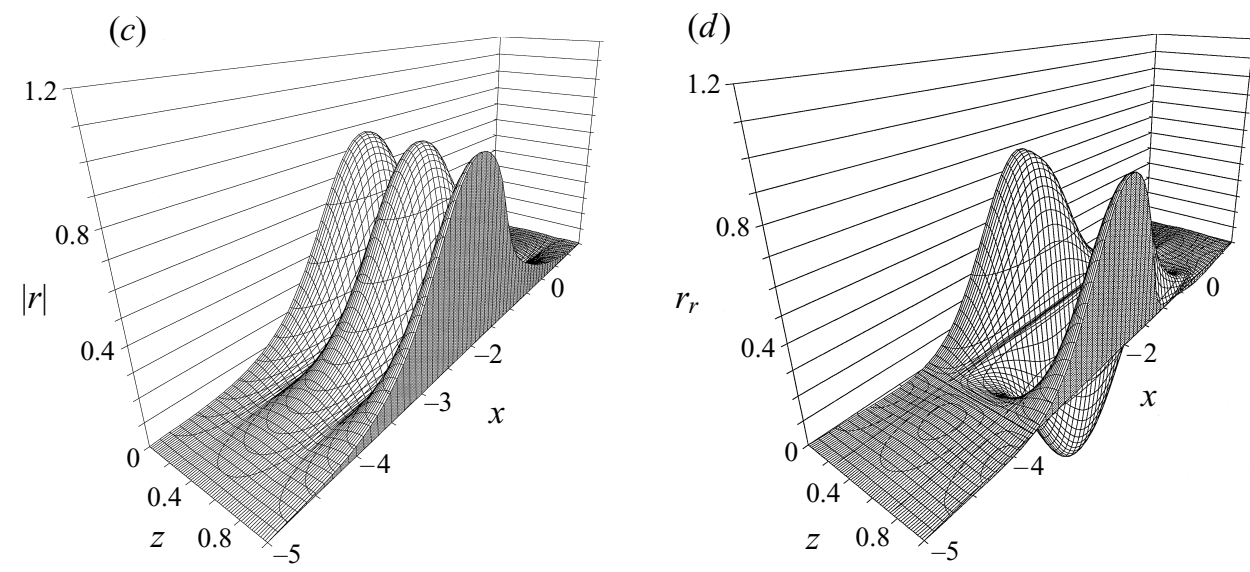

(e)
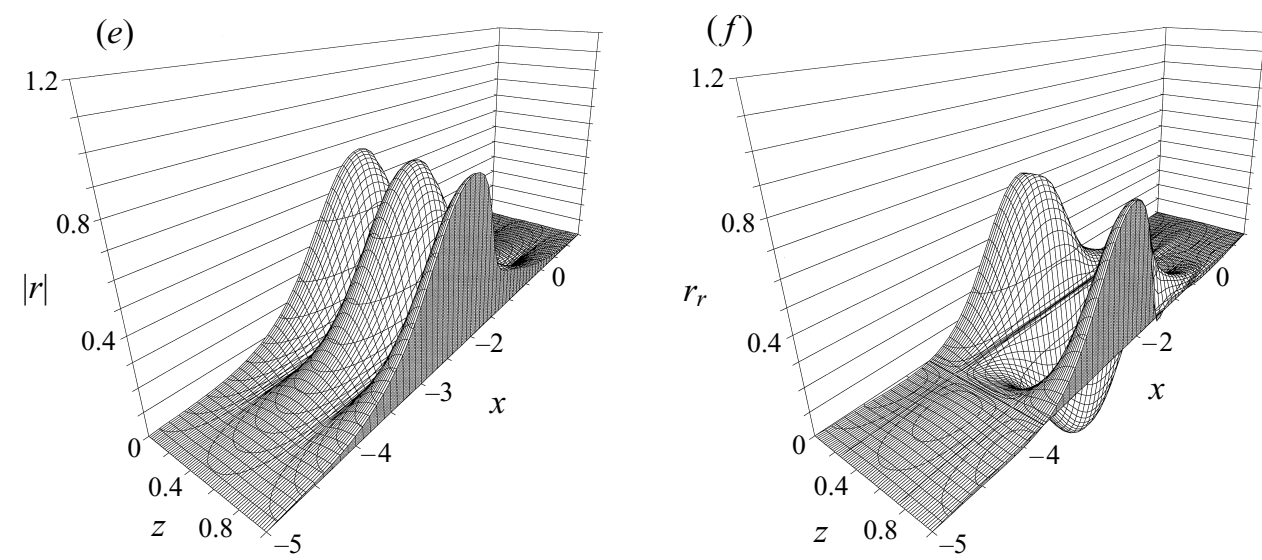

FIGURE $9(a-f)$. For caption see facing page. 

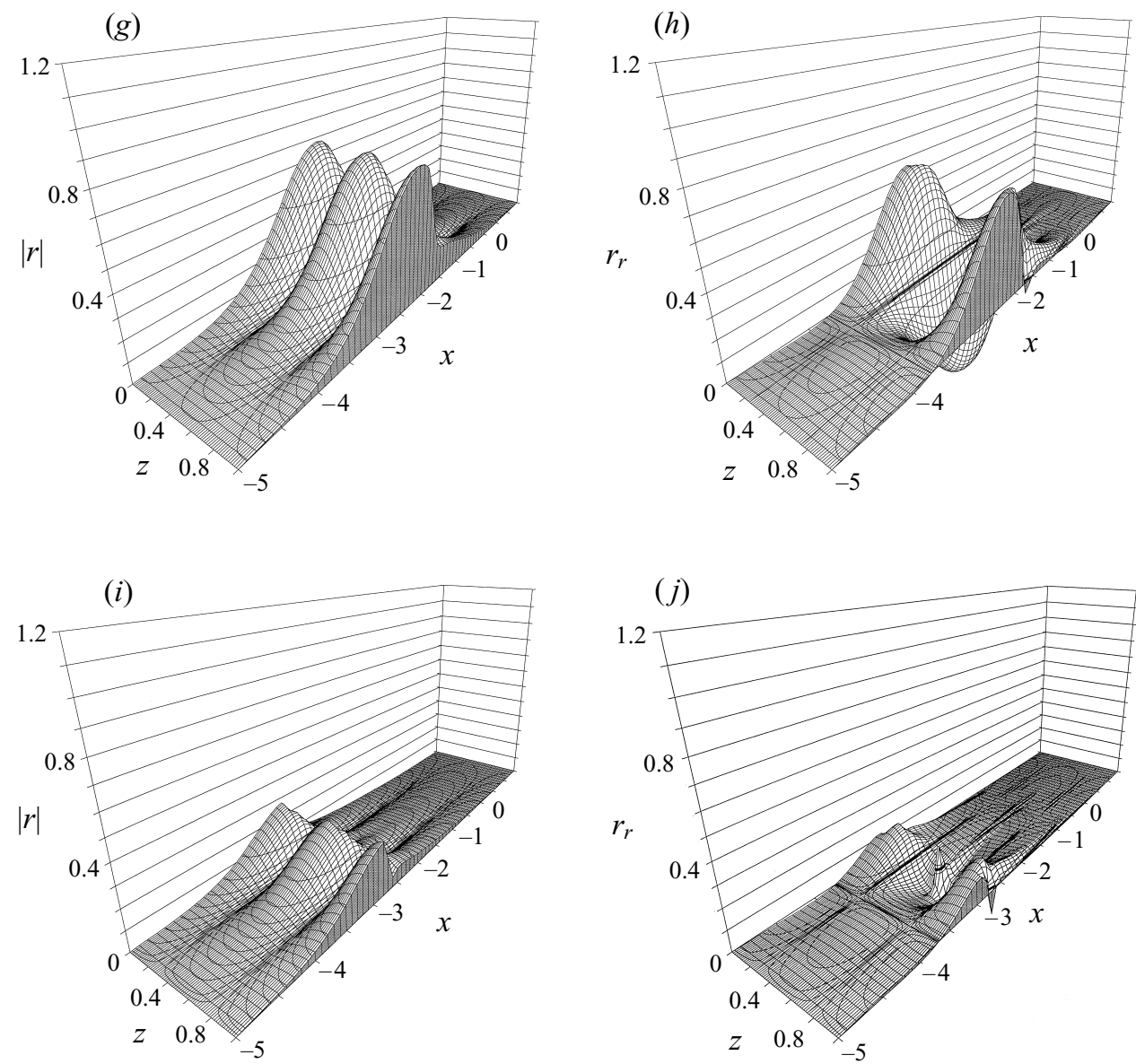

$(k)$
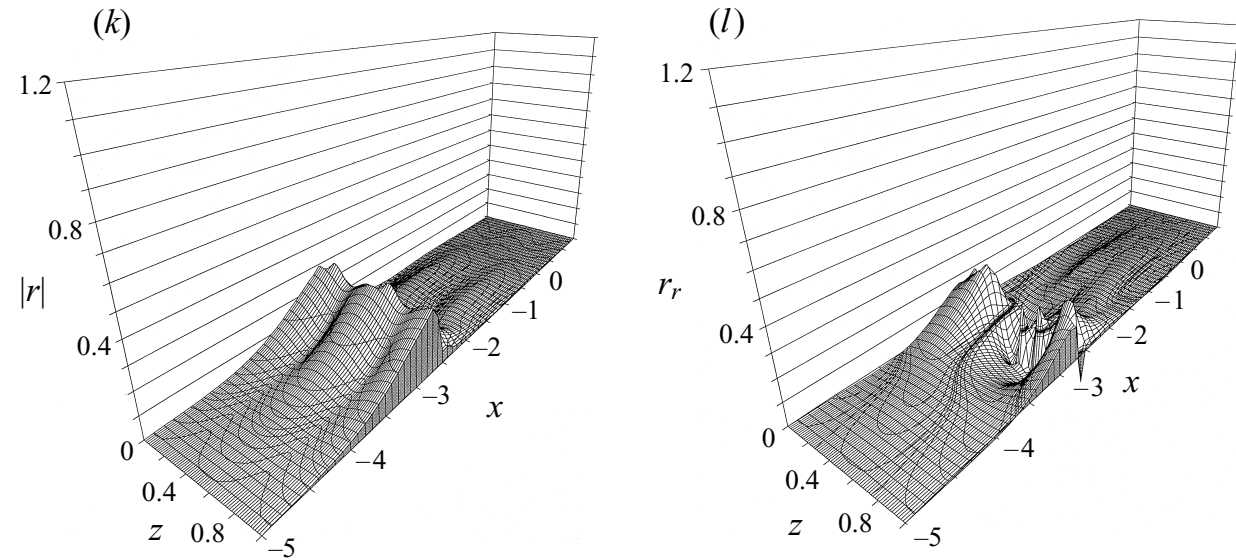

Figure 9. Nonlinear stabilization in a two-wave VWI, solution of (4.14), (4.39) with $a=-1+0.5 \mathrm{i}$, $b=0.02, c=-1, d=0, \beta=2 \pi$. $(a-j)$ The magnitude $|r|$ and the real part $r_{r}$ for various $e_{0}$ in the case of equal waves, $r_{\infty}^{(1)}=r_{\infty}^{(2)}=1 ;(a, b) e_{0}=0.02 ;(c, d) e_{0}=0.05 ;(e, f) e_{0}=0.075 ;(g, h) e_{0}=0.1$; $(i, j) e_{0}=1$ respectively; $(k, l)$ as above but for asymmetric input, $r_{\infty}^{(1)}=1, r_{\infty}^{(2)}=0.5$, and $e_{0}=1$. 

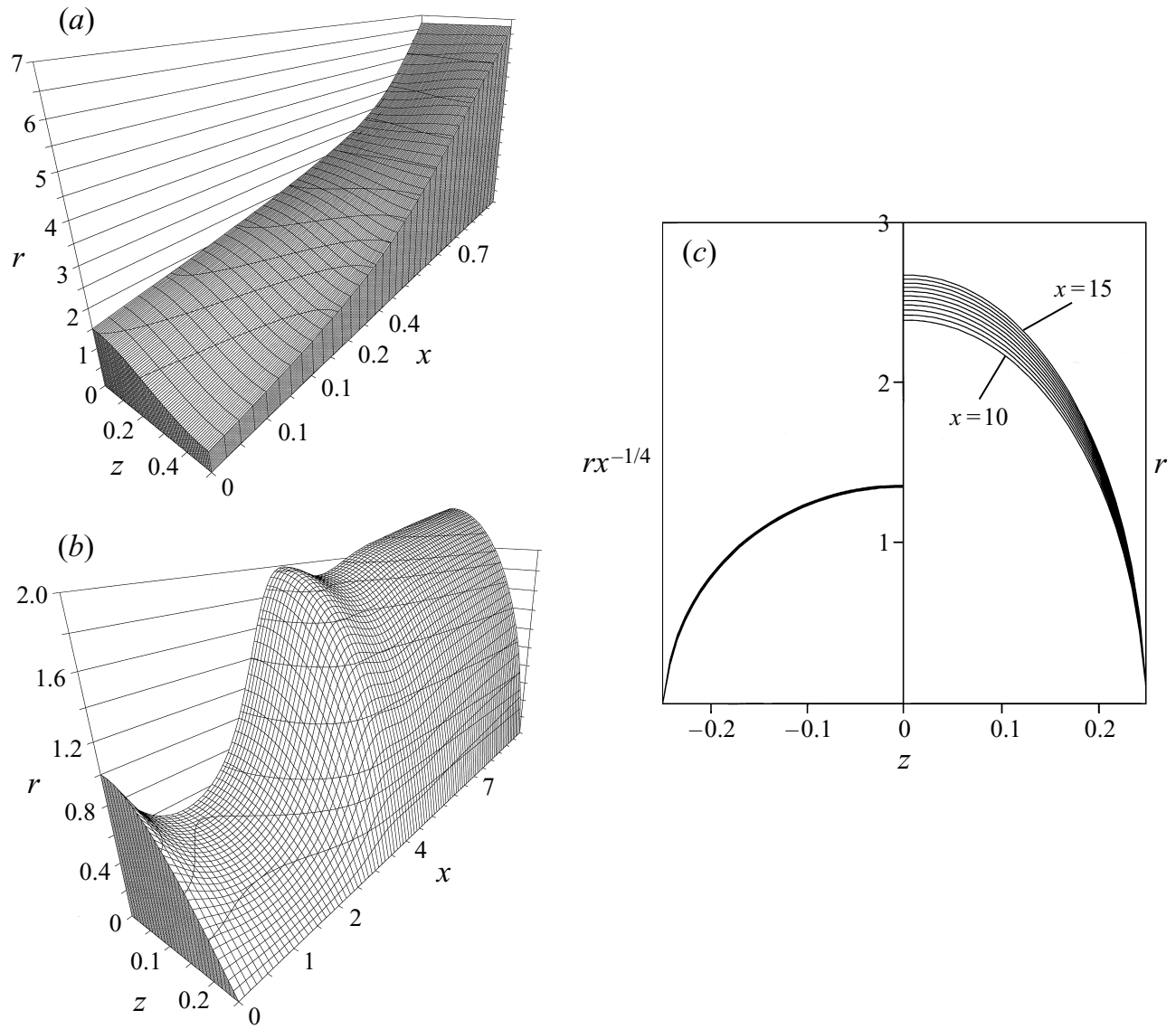

FIGURE $10(a-c)$. For caption see facing page.

interaction are notably small, a feature which is described in $\S 2$, and again as in that paper many solution paths are then found to be possible $(\$ \S 3,4)$, of which some are distinct from previous findings as mentioned in the following paragraphs.

Given a considerable degree of arbitrariness in the cross-scales of naturally or artificially excited perturbations in practical applications, we have attempted a complete account of the possible VWI regimes for disturbances significantly affected by the flow non-parallelism (a similar treatment can be given to temporal perturbations and to time-varying base flows). In addition to the Smith et al. (1993) flow with the non-dimensional cross-scale of $O\left(R^{-1 / 2}\right)$ two novel regimes are studied in this paper characterized by the spanwise dimension being increased to $O\left(R^{-23 / 48}\right)$ and $O\left(R^{-3 / 8}\right)$ in turn. In the first of these, studied in $\S 3$, the major novel feature, stemming originally from the classification in $\$ 2$, is the appearance of a convolution-integral term as a new extra nonlinear influence. It is due to the action, within the viscous critical layer, of a Reynolds-stress contribution distinct from that in Smith et al. (1993), the latter being associated with a jump produced in the spanwise mean shear across the critical layer whereas the convolution contribution comes from a jump induced in the mean spanwise velocity itself. The interaction flow properties match with those in the last-named paper if the typical $z$-scale is shortened, as expected. However in order to achieve the match the ratio of the cross-scales for the two regimes must be 

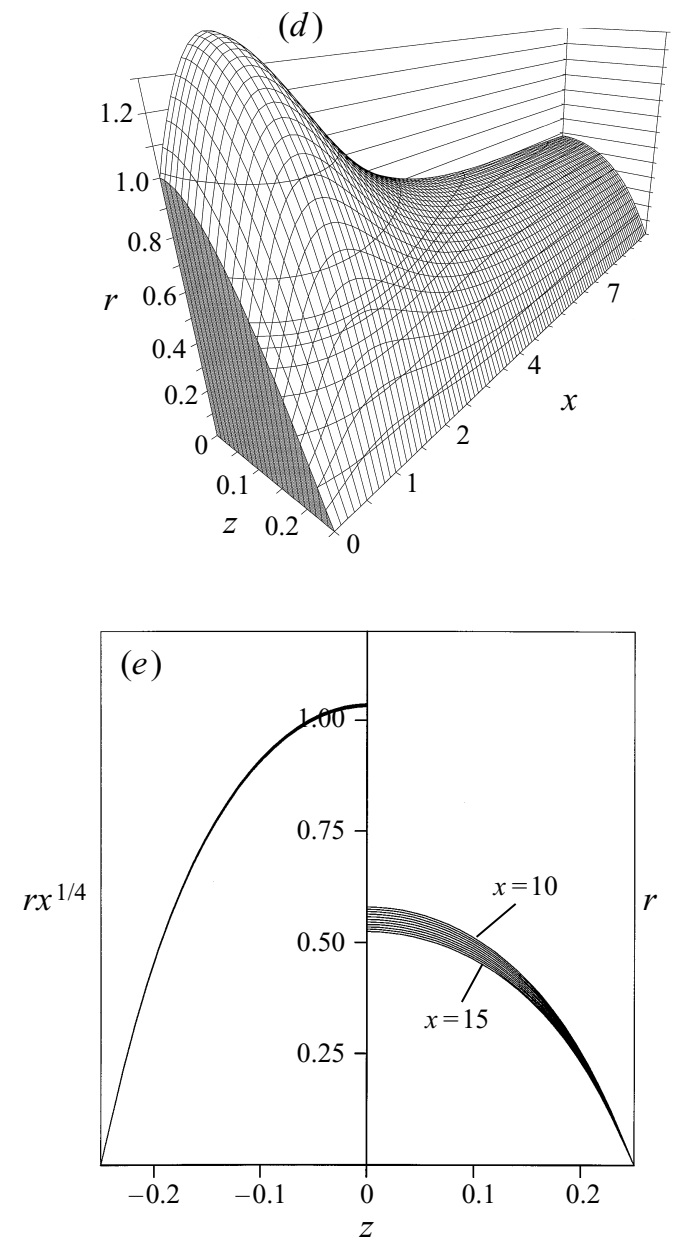

FIGURE 10. Nonlinear stabilization of a linearly unstable flow. $(a) r=1+0.5 \cos 2 \pi z$ at $x=0$, $a=-1, b=0.02, c=0, d=2, e_{0}=0.02 ;(b) r=\cos 2 \pi z$ at $x=0, a=-1, b=0.02, c=1, d=0$, $e_{0}=0.02 ;(c) r$ and $r x^{-1 / 4}$ vs. $z$ ( $-z$ on the left) from computations for $(b)$ plotted for $x$-values from 10 to 15 with step $\delta x=0.5 ;(d)$, as in (b) but with $c=0, d=2 ;(e) r$ and $r x^{1 / 4}$ vs. $z$ for $x$ varying from 10 to 15 with the step 0.5 , from the computation for $(d)$.

made small. Since this ratio is in fact proportional to $R^{-1 / 48}$, at realistic Reynolds numbers the convolution-integral term not present in Smith et al. (1993) may never be sufficiently small, and certain solution properties may become affected by this feature. For example, the solutions presented in $\$ 3$ show that with the convolution term maintained a substantial new phenomenon occurs, namely a 'stabilized' saturation in the far-downstream behaviour within that range of the controlling parameters where the shorter-scale analysis of Smith et al. (1993) predicts self-sustained oscillations.

It is interesting to note that, being based on the relatively large small parameter $R^{-1 / 48}$ and hence formally 'non-robust' (the term was suggested by one of the referees), the theory in $\S 3$ turns out to produce a more general and consequently more 'robust' final equation than the analysis in Smith et al. (1993) which relies on the smallness of the quantity $R^{-1 / 2}$. A more careful examination of the two regimes shows, however, that they should be treated as equally 'robust' in the sense that the properties of the two flows complement and enrich each other: the novel nonlinearity present in our $\S 3$ 

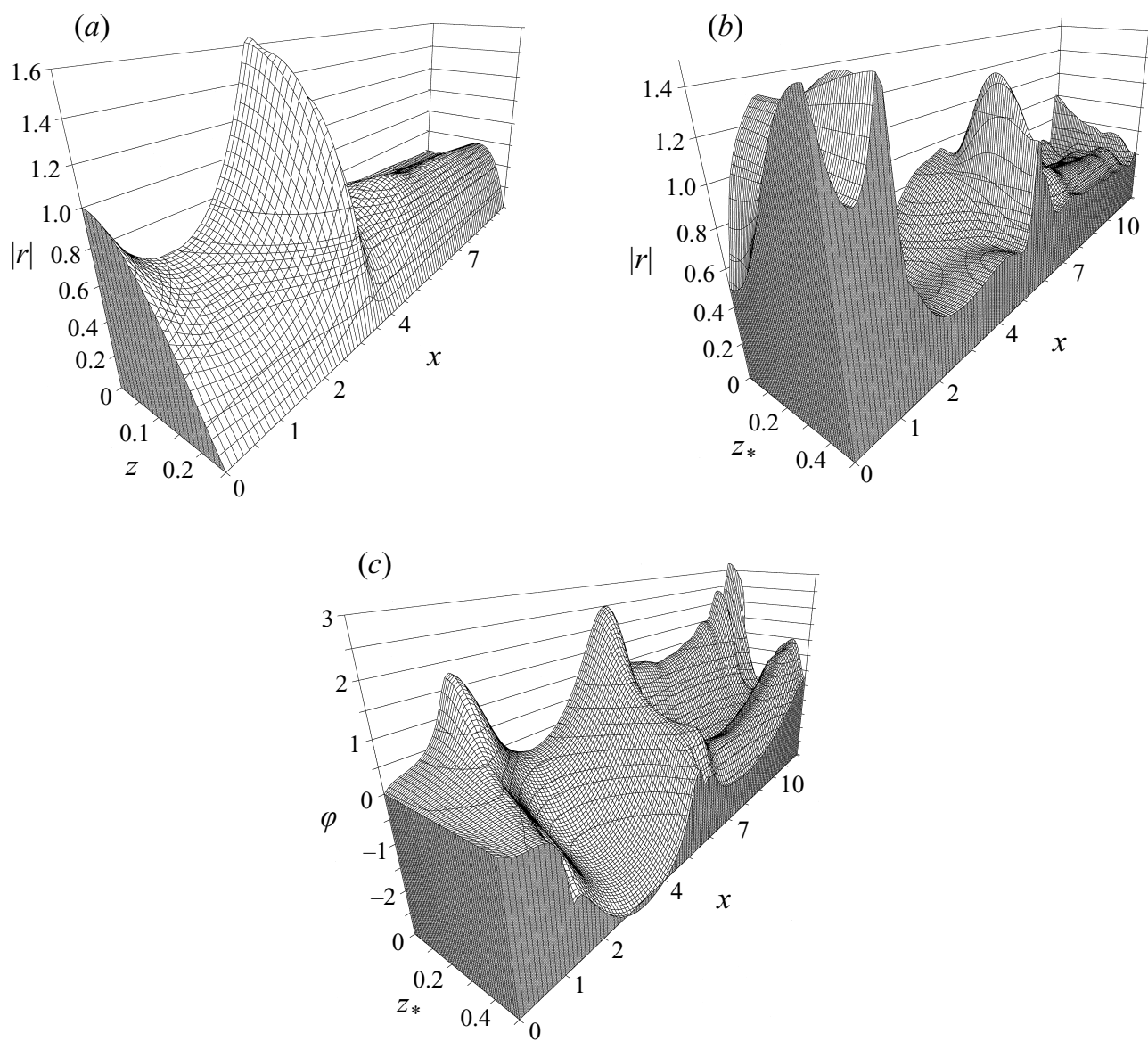

Figure 11. (a) $|r|$ from computations for (4.14) with the initial condition $r(x=0)=\cos 2 \pi z$ and $a=-1+0.5 \mathrm{i}, b=0.02, c=1, d=0, e_{0}=0.02$. $(b, c)$ The magnitude $|r|$ and phase $\varphi$ vs. $z_{*}=0.5-z$ in the case $r(x=0)=1+0.5 \cos 2 \pi z, a=-1+0.5 \mathrm{i}, b=0.08, c=1, d=0, e_{0}=0.08$.

is lacking in Smith et al. (1993) but instead the nonlinear constant in the last paper is able to change sign with the cross-wavenumber variation, a significant feature clearly absent in our case. The two regimes are therefore equally important. In the future they may serve as a convenient basis for a theory utilizing composite expansions and capturing the essential effects present in both cases.

The argument above is not limited to symmetric configurations. It would be interesting to see, for example, to what extent the nonlinear filtering mechanism described in $\S 3$ will be relevant to VWI with cross-flow studied in Brown \& Smith (1996), Allen et al. (1996).

The second regime of the VWI studied in $\S 4$ of this paper is dominated by the convolution-integral nonlinearity with partial crosswise derivatives encountered earlier in $\mathrm{Wu}(1993)$ and more recently in Wu et al. (1996). Accordingly the disturbance evolution is subject to strong mode exchanges and phase/amplitude interactions, linked directly with the secondary instability phenomena; cf. e.g. Hocking \& Stewartson (1972), Hocking, Stewartson \& Stuart (1972). All this has a profound impact on the flow properties. In particular, the nonlinear saturation downstream becomes less probable for it requires a very careful arrangement of the base flow and input perturbations so as to eliminate even slightest phase variations in the disturbance. 
More natural routes in the wave development include a decay, a finite-distance selffocusing also present in the special case considered by Wu et al. (1996), and a complex finite-amplitude (probably quasi-periodic) modulation.

The analysis in this paper may seem somewhat formal in that no particular flow has been chosen to calculate the specific coefficients in the evolution equations. This was done deliberately, first with the aim of presenting the theory in the most general form, and secondly in order to try to reveal the most typical routes of the flow development which, as previous studies tend to indicate, are determined primarily by the signs of the constants in the controlling formulation rather than the particular values of these constants (this is only true for relatively simple cases, see e.g. the influence of the mean Fourier component on both real-valued and complex solutions in $\S 4$ ). The few representative cases tackled in this work and in related studies provide a foundation for future comparisons with experiments.

Overall then, the theory in this paper and in Smith et al. (1993) covers lowamplitude, non-parallel regimes of VWI in Rayleigh-unstable planar flows. Analogous regimes for stronger waves and wave packets governed by viscous nonequilibrium critical-layer equations are considered in Wu et al. (1993) and Wu (1993), whereas Goldstein \& Choi (1989) considered previously the corresponding development of even stronger inviscid waves with non-equilibrium critical layers. Whilst non-equilibrium critical-layer regimes for these and other configurations tend to be associated with explosive growth of participating disturbances (see references in the Introduction), the milder, lower-input flows with VWI can be divided loosely into three categories: (i) strongly stabilized flows with the ultimate decay of input disturbances, (ii) strongly destabilized regimes which terminate at a finite point in space or time and eventually develop into a non-equilibrium critical-layer stage, and (iii) the regimes leading to the origin of 'complex' behaviour. Various forms of complex behaviour arise typically when the flow non-parallelism enhances the linear instability on the background of damping nonlinear mechanisms as, for instance, in a boundary layer under a progressively increasing adverse pressure forcing or near wall-mounted obstacles; see Smith et al. (1993), Timoshin (1996). For such regimes Smith et al. (1993) indicate the appearance of periodic modulations degenerating under special conditions into almost solitary peaks of the amplitude envelope. Brown \& Smith (1996) report the occurrence of quasi-periodic and highly irregular solutions. Our analysis in $\$ 3$ points to the existence of nonlinear filtering, and rather complex solutions were encountered in $\S 4$ (see also Wu 1993). The large-scale complexity above should not be mistaken for laminar-turbulent transition itself, the important outcome being however the development of nonlinear temporal and spatial structures and scales not present in the original unperturbed flow.

The authors are grateful to Professor S. N. Brown for a number of interesting discussions, to the referees for their comments, and to The Nuffield Foundation for support for S. N. T.

\section{Appendix A}

The normal velocity component $v^{(1)}$ in (3.3) is governed by

$$
\begin{gathered}
\left(U_{0}-c_{0}\right)\left(\frac{\partial^{2} v^{(1)}}{\partial \bar{y}^{2}}-\alpha_{0}^{2} v^{(1)}\right)-U_{0}^{\prime \prime} v^{(1)}=\varphi(\bar{y})\left[\frac{\mathrm{i} \alpha_{0}}{b_{1}}\left(U_{0}-c_{0}\right)\left(\frac{\partial^{2} r}{\partial Z^{2}}-2 \alpha_{0} \alpha_{1} r\right)\right. \\
\left.+\frac{\mathrm{i} \alpha_{1}}{b_{1}}\left(c_{0}-c_{1}\right) \frac{U_{0}^{\prime \prime}}{U_{0}-c_{0}}\right] ; v^{(1)}=0 \text { at } \bar{y}=0 \text { and as } \bar{y} \rightarrow \infty .
\end{gathered}
$$


Since $v^{(1)}$ and $\partial v^{(1)} / \partial \bar{y}$ must be continuous at $\bar{y}_{c}$ the forcing term in the equation vanishes, hence

$$
\partial^{2} r / \partial Z^{2}=2 \alpha_{0} \alpha_{1} r \text { and } c_{1}=\Omega_{1} / \alpha_{1}=c_{0} .
$$

For similar reasons in the next approximations we have $\Omega_{m}=c_{0} \alpha_{m}$ for $m=0,1,2 \ldots$. Also, since $\alpha_{0}, \alpha_{1}, \ldots$ are assumed to be real, we take $2 \alpha_{0} \alpha_{1}=-\beta^{2}$, where the real $\beta$ corresponds to the $Z$-wavenumber of the disturbances.

The subsequent terms in the core expansions are governed by the equations

$$
\begin{gathered}
\mathrm{i}\left(U_{0}-c_{0}\right)\left[\alpha_{0} u^{(m)}+\alpha_{1} u^{(m-1)}+\ldots+\alpha_{m} u^{(0)}\right]+U_{0}^{\prime} v^{(m)} \\
+\mathrm{i}\left[\alpha_{0} p^{(m)}+\alpha_{1} p^{(m-1)}+\ldots+\alpha_{m} p^{(0)}\right]=0, \\
\mathrm{i}\left(U_{0}-c_{0}\right)\left[\alpha_{0} v^{(m)}+\alpha_{1} v^{(m-1)}+\ldots+\alpha_{m} v^{(0)}\right]+\frac{\partial p^{(m)}}{\partial \bar{y}}=0, \\
\mathrm{i}\left(U_{0}-c_{0}\right)\left[\alpha_{0} w^{(m)}+\alpha_{1} w^{(m-1)}+\ldots+\alpha_{m} w^{(0)}\right]+\frac{\partial p^{(m)}}{\partial Z}=0, \\
\mathrm{i}\left[\alpha_{0} u^{(m)}+\alpha_{1} u^{(m-1)}+\ldots+\alpha_{m} u^{(0)}\right]+\frac{\partial v^{(m)}}{\partial \bar{y}}+\frac{\partial w^{(m-1)}}{\partial Z}=0,
\end{gathered}
$$

with $m=1,2, \ldots$. A particular solution is sought in the form

$$
\begin{gathered}
v^{(m)}=A_{m} \varphi(\bar{y}), p^{(m)}=B_{m}\left[U_{0}^{\prime} \varphi-\left(U_{0}-c_{0}\right) \varphi^{\prime}\right], \\
w^{(m)}=C_{m}\left[U_{0}^{\prime} \varphi-\left(U_{0}-c_{0}\right) \varphi^{\prime}\right] /\left(U_{0}-c_{0}\right),
\end{gathered}
$$

where the coefficients $A_{m}, B_{m}, C_{m}$ satisfy the equations

$$
\begin{gathered}
\alpha_{0} A_{m}+\alpha_{1} A_{m-1}+\ldots+\alpha_{m} A_{0}=0, \\
\frac{\partial C_{m-1}}{\partial Z}=A_{m}+\mathrm{i} \alpha_{m} B_{0}, \\
\alpha_{0} C_{m-1}+\alpha_{1} C_{m-2}+\ldots+\alpha_{m-1} C_{0}=0,
\end{gathered}
$$

assuming that a solution exists with $B_{0} \neq 0, B_{m}=0$ for $m>0$. Since $C_{0}=$ $\mathrm{i} \alpha_{0}^{-1} \partial B_{0} / \partial Z, A_{0}=-\mathrm{i} \alpha_{0} B_{0}$, the sequence of equations (A 9)-(A 11) provides the values of $A_{m}, C_{m}$ and the wavenumber correction terms $\alpha_{m}$ successively for $m=1,2, \ldots$ as can be shown inductively.

Near the critical level $\bar{y}=\bar{y}_{c}$ the normal velocities and the pressure functions are regular at $\bar{y}_{c}$, and so is the main streamwise velocity $u^{(0)}$ in (3.6). However the higherorder terms $u^{(1)}, u^{(2)}, \ldots$, and all the terms in the cross-velocity expansion contain a simple pole singularity in view of (A 6) and (A 8).

\section{Appendix B}

In the viscous critical layer (CL) for the flow in $\S 3$ we define $y=\varepsilon^{24}\left(\bar{y}_{c}+\varepsilon^{8} y_{2}\right)$, with $y_{2}=O(1)$. The flow functions expand in the form

$$
\begin{gathered}
u=c_{0}+\varepsilon^{8} b_{1} y_{2}+\ldots+\varepsilon^{20}\left\{E\left[\bar{u}_{0}+\ldots+\varepsilon^{18} \ln \varepsilon \bar{u}_{1,0}+\varepsilon^{18} \bar{u}_{1}+\ldots\right]+\text { c.c. }\right\}, \\
v=\varepsilon^{24} \bar{V}_{0}+\ldots+\varepsilon^{26}\left\{E\left[\bar{v}_{0}+\varepsilon^{2} \bar{v}_{1}+\ldots+\varepsilon^{20} \ln \varepsilon \bar{v}_{2,0}+\varepsilon^{20} \bar{v}_{2}+\ldots\right]+\text { c.c. }\right\}, \\
w=\varepsilon^{29} \ln \varepsilon \bar{W}_{0,0}+\varepsilon^{29} \bar{W}_{0}+\varepsilon^{31} \bar{W}_{1}+\ldots+\varepsilon^{19}\left\{E\left[\bar{w}_{0}+\varepsilon^{2} \bar{w}_{1}+\ldots\right]+\text { c.c. }\right\}, \\
p=p_{0}+\ldots+\varepsilon^{26}\left\{E\left[\bar{p}_{0}+\varepsilon^{2} \bar{p}_{1}+\ldots\right]+\text { c.c. }\right\},
\end{gathered}
$$


with $\bar{p}_{0}=r$. The cross-velocities in the wave are given by

$$
\begin{gathered}
\bar{w}_{0}=\frac{\partial r}{\partial Z} \Psi\left(y_{2}\right), \Psi\left(y_{2}\right)=-\left|\alpha_{0} b_{1}\right|^{-2 / 3} \int_{0}^{\infty} \exp \left[-\operatorname{isgn}\left(\alpha_{0} b_{1}\right)\left|\alpha_{0} b_{1}\right|^{1 / 3} s-s^{3} / 3\right] \mathrm{d} s, \\
\bar{w}_{1}=\left(\frac{\partial \bar{p}_{1}}{\partial Z}-\frac{2}{3} \frac{\alpha_{1}}{\alpha_{0}} \frac{\partial r}{\partial Z}\right) \Psi\left(y_{2}\right)+\frac{\mathrm{i}}{3} \frac{\alpha_{1}}{\alpha_{0}}\left|\alpha_{0} b_{1}\right|^{-2 / 3} \frac{\partial r}{\partial Z} y_{2} \\
\times \int_{0}^{\infty} s \exp \left[-\operatorname{isgn}\left(\alpha_{0} b_{1}\right)\left|\alpha_{0} b_{1}\right|^{1 / 3} y_{2} s-s^{3} / 3\right] \mathrm{d} s .
\end{gathered}
$$

The streamwise momentum and the continuity equations indicate that

$$
\bar{v}_{0}=-\mathrm{i} \alpha_{0} b_{1}^{-1} r, \quad \bar{v}_{1}=-\mathrm{i}\left(\alpha_{0} \bar{p}_{1}+\alpha_{1} r\right) b_{1}^{-1}, \quad \bar{u}_{0}=\frac{\mathrm{i}}{\alpha_{0}} \frac{\partial^{2} r}{\partial Z^{2}} \Psi\left(y_{2}\right) .
$$

The formulae above serve to smooth out the pole singularity in the inviscid outer solution. The removal of the weaker logarithmic singularity is achieved in the terms $\bar{u}_{1}, \bar{v}_{2}$ in (B 1), (B 2). Elimination of passive forcing terms leads to the formulation for $\bar{v}_{2}$ of the form

$$
\begin{gathered}
\bar{v}_{2}-y_{2} \frac{\partial \bar{v}_{2}}{\partial y_{2}}+\left(Q_{1}+Q_{i}\right) y_{2}=\frac{\mathrm{i}}{\alpha_{0} b_{1}} \frac{\partial^{3} \bar{v}_{2}}{\partial y_{2}^{3}}, \\
\bar{v}_{2}=\left(Q_{1}+Q_{i}\right) y_{2} \ln \left|y_{2}\right|+\left(A^{ \pm}-Q_{1}-Q_{i}\right) y_{2}+\ldots, \text { as } y_{2} \rightarrow \pm \infty .
\end{gathered}
$$

The coefficients in (B 8) are introduced in $\$ 3$. The problem posed, which is now classical in the linear stability theory, leads immediately to (3.38).

The vortex-flow generation within the $\mathrm{CL}$ is contained in the cross-momentum balances. For the first three mean-flow terms in (B 3) we have the equations

$$
\begin{gathered}
\frac{\partial^{2} \bar{W}_{0,0}}{\partial y_{2}^{2}}=0, \quad \frac{\partial^{2} \bar{W}_{0}}{\partial y_{2}^{2}}=\bar{v}_{0}^{*} \frac{\partial \bar{w}_{0}}{\partial y_{2}}+\text { c.c. } \\
\frac{\partial^{2} \bar{W}_{1}}{\partial y_{2}^{2}}=-\mathrm{i} \alpha_{0}\left(\bar{u}_{0} \bar{w}_{0}^{*}-\text { c.c. }\right)+\left(\bar{v}_{1}^{*} \frac{\partial \bar{w}_{0}}{\partial y_{2}}+\bar{v}_{0}^{*} \frac{\partial \bar{w}_{1}}{\partial y_{2}}+\bar{w}_{0}^{*} \frac{\partial \bar{w}_{0}}{\partial Z}+\text { c.c. }\right) .
\end{gathered}
$$

The $y_{2}$-independent term $\bar{W}_{0,0}$ has no impact on the solution. Integrating the second equation in (B 10) and equation (B 11) using (B 5)-(B 7), we obtain the jump conditions

$$
\begin{gathered}
{\left[\bar{W}_{0}+b_{1}^{-2} \frac{\partial}{\partial Z}\left(|r|^{2} \ln \left|y_{2}\right|\right)\right]_{-\infty}^{+\infty}=J_{0},} \\
{\left[\frac{\partial \bar{W}_{1}}{\partial y_{2}}\right]_{-\infty}^{+\infty}=J_{1},}
\end{gathered}
$$

with the values of the jumps given explicitly in (3.32), (3.33). Rewritten in terms of the buffer-layer variable $y_{1}=y_{2} \varepsilon^{-2}$, the relations (B 12), (B 13) lead to (3.31).

\section{Appendix C}

A weakly nonlinear version of the amplitude equation (3.46) can be derived if the flow is close to the saturated state with $\rho=1$ for all $\xi>0$. The equality is exact if we take $\tilde{c}=-1, \tilde{e}_{0}=0, \tilde{e}_{1}=-1$ in (3.46), assuming also that $\xi_{0}=0$. Changes in $\tilde{e}_{1}$ result in periodic oscillations of the amplitude around $\rho=1$, cf. Smith et al. 
(1993), whereas non-zero negative values of $\tilde{e}_{0}$ tend to restore the saturated form far downstream; see $\$ 3$ and below.

Instead of altering the coefficient $\tilde{e}_{1}$ we may perturb the value of $\tilde{e}_{0}$ taking $\rho(0)=$ $1+\varepsilon, \tilde{e}_{0}=-\delta_{0} \varepsilon^{2}, \delta_{0}=O(1)>0$, and $\varepsilon \ll 1$. The solution is then sought in the form

$$
\rho=1+\varepsilon \rho_{1}(\xi, \zeta)+\varepsilon^{2} \rho_{2}(\xi, \zeta)+\varepsilon^{2} \rho_{3}(\xi, \zeta)+\ldots,
$$

where $\zeta=\xi \varepsilon^{2}$ is the slow variable. The first correction term is given by

$$
\rho_{1}=A_{1} \exp (\mathrm{i} \xi)+\text { c.c., } \quad A_{1}(0)+A_{1}^{*}(0)=1,
$$

with the last constraint following from the initial condition. Since

$$
\begin{aligned}
\int_{0}^{\xi} \rho_{1}\left(s, s \varepsilon^{2}\right) \mathrm{d} s= & \frac{1}{\mathrm{i}} \exp (\mathrm{i} \xi) A_{1}(\zeta)-\frac{1}{\mathrm{i}} A_{1}(0) \\
& +\varepsilon^{2}\left[\exp (\mathrm{i} \xi) A_{1}^{\prime}(\zeta)-A_{1}^{\prime}(0)\right]+O\left(\varepsilon^{4}\right)+\text { c.c. as } \varepsilon \rightarrow 0,
\end{aligned}
$$

we obtain that $A_{1}(0)=A_{1}^{*}(0)$. Also the expansion

$$
\int_{0}^{\xi} \rho \frac{\mathrm{d} s}{(\xi-s)^{1 / 2}}=2 \xi^{1 / 2}+\varepsilon\left\{\pi^{1 / 2} \exp \left[\mathrm{i}\left(\xi-\frac{1}{4} \pi\right)\right] A_{1}(\zeta)+\text { c.c. }\right\}+O\left(\varepsilon^{2}\right)
$$

holds for the first integral in (3.46). We will show that the phase shift $\pi / 4$ in the oscillatory term in $(\mathrm{C} 4)$ has a stabilizing influence on the downstream wave behaviour. The $\varepsilon^{2}$ contribution in (C1) is found to be $\rho_{2}=2 A_{1}^{2} \exp (2 \mathrm{i} \xi) / 3+$ c.c., omitting insignificant main harmonics $\exp ( \pm \mathrm{i} \xi)$. The solvability requirement for $\rho_{3}$ in the next approximation then yields the Landau-Stuart equation,

$$
A_{1}^{\prime}=-\frac{\mathrm{i}}{6} A_{1}\left|A_{1}\right|^{2}-\delta_{0} \pi^{1 / 2} \mathrm{e}^{-\mathrm{i} \pi / 4} A_{1}
$$

for the slow-scale wave modulation. If, first, $\delta_{0}=0$ then

$$
A_{1}=A_{1}(0) \exp \left[-\mathrm{i}\left|A_{1}(0)\right|^{2} \zeta / 6\right],
$$

and hence the modulated wave remains purely periodic with the period dependent on the amplitude. If, however, $\delta_{0}>0$ then $\left|A_{1}\right|^{2}=\left|A_{1}(0)\right|^{2} \exp \left(-\delta_{0}(2 \pi)^{1 / 2} \zeta\right)$, with $\left|A_{1}\right|^{2} \rightarrow 0$ as $\zeta \rightarrow \infty$. The two options correspond to the neutral stability of saturated VWI in Smith et al. (1993) and to stable saturation far downstream in our computations in $\S 3$, in turn.

\section{Appendix D}

The equation of the form

$$
a \frac{\partial r}{\partial x}+b \frac{\partial^{2} r}{\partial z^{2}}+Q \frac{\cos \beta z}{(x+1)^{\alpha}} r=0
$$

can be regarded as a model of the far-downstream limit equation (4.31). Our concern here is with the asymptotic behaviour of $z$-periodic solutions of (D1) at large $x$. The initial condition is chosen in the form $r(x=0, z)=1+N \cos \beta z$, as a representative example; the constants $\alpha, \beta, b$ are real, with $b>0$, whereas $Q, a, N$ can be complex but the real part $a_{r}$ is negative, so that the solution with $Q=0$ given by $r=r_{0}(x, z)=1+N \mathrm{e}^{\kappa x} \cos \beta z$, with $\kappa=b \beta^{2} a^{-1}$, has the limit property $r_{0} \rightarrow 1$ as 
$x \rightarrow \infty$, on account of $\kappa_{r}<0$. If, first, $|Q| \ll 1$ then a direct calculation shows that

$$
\begin{aligned}
r= & 1+\text { T.S.T. }+Q\left[r_{10}(\infty)+\frac{\cos \beta z}{b \beta^{2}(1+x)^{a}}+\text { T.S.T. }\right] \\
& +Q^{2}\left[-\frac{1}{2 a b \beta^{2}} \frac{(1+x)^{1-2 \alpha}}{1-2 \alpha}+r_{20}(\infty)+\frac{r_{10}(\infty)}{b \beta^{2}} \frac{\cos \beta z}{(1+x)^{\alpha}}\right. \\
& \left.-\frac{1}{2 b \beta^{2}} \frac{\cos 2 \beta z}{(1+x)^{2 \alpha}}+\text { T.S.T. }\right]+\ldots \text { as } x \rightarrow \infty,
\end{aligned}
$$

where T.S.T. denotes exponentially small terms, and $r_{10}(\infty), r_{20}(\infty)$ are constants. Hence when $\alpha>1 / 2$ the non-zero Fourier components decay algebraically leaving the limit solution in the form $r \rightarrow 1+Q r_{10}(\infty)+Q^{2} r_{20}(\infty)+\ldots$, as $x \rightarrow \infty$. This type of behaviour was observed in $\$ 4$ in the case of real solutions, see figure $5(b)$. In the range $0<\alpha<1 / 2$ the Fourier harmonics exhibit algebraic decay, however the expansion in powers of $Q$ fails at larger $x$ because of the growing mean of order $Q^{2}(1+x)^{1-2 \alpha}$. Hence we define $\xi=(1+x) L^{-1}=O(1)$, with $L \gg 1$, and expand the solution in the form

$$
r=R_{0}(\xi)+\ldots+\frac{Q}{L^{\alpha}} R_{\alpha}(\xi) \cos \beta z+\ldots+\frac{1}{L} R_{L}(\xi, z)+\ldots,
$$

assuming that $Q^{2} L^{1-2 \alpha}=O(1)$ and that the omitted terms are ordered in accordance with the particular value of $\alpha$. Substitution into (D 1) yields the two relations

$$
R_{\alpha}=\frac{1}{b \beta^{2} \xi^{\alpha}} R_{0}(\xi), \quad a \frac{\mathrm{d} R_{0}}{\mathrm{~d} \xi}+b \frac{\partial^{2} R_{L}}{\partial z^{2}}+\frac{\cos ^{2} \beta z}{b \beta^{2} \xi^{2 \sigma}} R_{0}=0 .
$$

The requirement of $z$-periodicity for $R_{L}$ and the match condition $R_{0}(0)=1$ yield the solution $R_{0}(\xi)=\exp \left[-\left(2 a b \beta^{2}\right)^{-1}(1-2 \alpha)^{-1} \xi^{1-2 \alpha}\right]$. It is important to note that a formally small term added to the conventional heat equation in (D 1) gives rise to exponentially growing solutions in the range $0<\alpha<1 / 2$. In the case $\alpha=1 / 2$ the expansion (D 3) is replaced by

$$
\begin{aligned}
r=1+\ldots+Q & {\left[r_{10}(\infty)+b^{-1} \beta^{-2}(1+x)^{-1 / 2} \cos \beta z+\ldots\right] } \\
& +Q^{2}\left[-(2 a b)^{-1} \beta^{-2} \ln (x+1)+r_{20}(\infty)+\ldots\right]+\ldots,
\end{aligned}
$$

with a growing mean term present at order $Q^{2}$. The required improved solution at larger $x$ can be derived directly from (D 1) assuming that $Q$ is finite initially. For then

$$
r=\frac{A_{0}(Q)}{(x+1)^{\gamma}}+\frac{A_{1}(Q) \cos \beta z}{(x+1)^{\gamma+1 / 2}}+\frac{A_{2}(z, Q)}{(x+1)^{\gamma+1}}+\ldots \quad x \gg 1,
$$

where $\gamma$ depends on $Q$. In addition $A_{1}=A_{0} Q\left(b \beta^{2}\right)^{-1}, 2 a A_{0} \gamma=Q A_{1}$, from the balance between the last two terms in (D 1$)$ and from the solvability for $A_{2}(z, Q)$ respectively. Hence $\gamma=Q^{2}\left(2 a b \beta^{2}\right)^{-1}$. In the additional limit $|Q| \rightarrow 0$ we have $A_{0}(Q) \rightarrow 1$ on account of (D 5), (D 6), whereas $(x+1)^{\gamma}$ with $\gamma \rightarrow 0$ produces a sequence of logarithmic terms.

We conclude that the solution of (D 1) with $\alpha=1 / 2$ develops an algebraic asymptote of the form (D6) as $x \rightarrow \infty$. The real part of $\gamma$ can be positive or negative depending on the particular values of the complex parameters $a$, $Q$, hence the solution can be growing or decaying, as illustrated in figure 12. In computations for the nonlinear VWI in $\$ 4$ the wave growth is suppressed by the nonlinearity, in consequence the real part of $\gamma$ was found to be positive. 


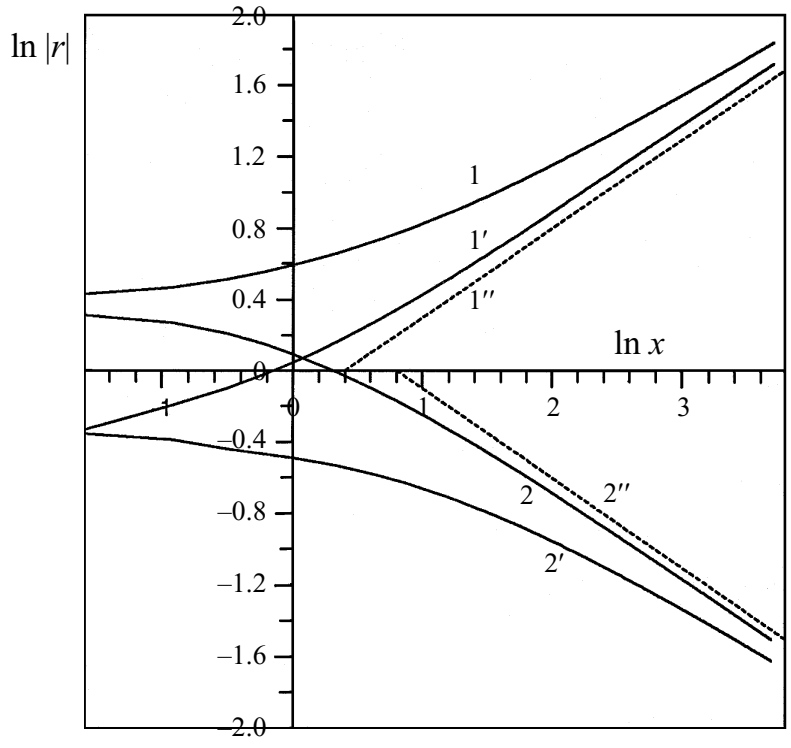

FIGURE 12. Numerical solutions of equation (D 1) with $\alpha=0.5, N=0.5, b=0.2, a=-1+0.5 \mathrm{i}$, $\beta=2 \pi$; also $Q=\pi(1+\mathrm{i} \Gamma)$, where $\Gamma=1(\gamma=0.5-\mathrm{i})$ for the curves $1,1^{\prime}$ corresponding to $z=0,0.5$ respectively, and $\Gamma=0(\gamma=-0.5-0.25 \mathrm{i})$ for the decaying solution $2,2^{\prime}$ at the same sections. The straight lines $1^{\prime \prime}, 2^{\prime \prime}$ drawn with the slopes $0.5,-0.5$ respectively illustrate the approach to the asymptote (D 6).

\section{Appendix E}

The far-downstream decay of the complex-valued pressure function observed in computations for the VWI in $\S 4$ with linearly unstable waves will be illustrated here in the analysis of limit solutions in narrowing zones surrounding the lines of zero wave pressure; see figure $11(a), z=0.25$. The balance of the second spanwise derivative with the linear growth term and nonlinear integral in (4.14) suggests the expansion

$$
r=x^{-1 / 4} \hat{\rho}(\zeta) \exp \left[\mathrm{i}\left(\frac{1}{2} \lambda x^{2}+\hat{\varphi}(\zeta)+\ldots\right)\right]+\ldots,
$$

as $x \rightarrow \infty, \zeta=\left(z-z_{c}\right) x^{1 / 2}=O(1) ; \lambda$ is a constant and $z=z_{c}$ corresponds to the line of zero pressure. The real amplitude and phase of the wave pressure, $\hat{\rho}$ and $\hat{\varphi}$ respectively, are governed by the equations

$$
\begin{gathered}
a_{1} \hat{\rho}+b\left[\hat{\rho}^{\prime \prime}-\hat{\rho}\left(\hat{\varphi}^{\prime}\right)^{2}\right]+2 e_{0} \frac{\hat{\rho}}{\zeta^{2}} \int_{0}^{\zeta}\left(\hat{\rho} \hat{\rho}^{\prime}\right)^{\prime} \frac{t^{2} \mathrm{dt}}{\left(\zeta^{2}-t^{2}\right)^{1 / 2}}=0, \\
a_{0} \hat{\rho}+\frac{b}{\hat{\rho}}\left(\hat{\rho} \hat{\varphi}^{\prime}\right)^{\prime}-\frac{2 e_{0} \hat{\rho}}{\zeta^{2}} \int_{0}^{\zeta}\left(\hat{\rho}^{2} \hat{\varphi}^{\prime}\right)^{\prime} \frac{t^{2} \mathrm{~d} t}{\left(\zeta^{2}-t^{2}\right)^{1 / 2}}=0
\end{gathered}
$$

where $a_{1}=c_{r}-\lambda a_{i}, a_{0}=c_{i}+\lambda a_{r}$. The initial conditions are taken in the form $\hat{\rho}(0)=0, \hat{\varphi}^{\prime}(0)=0$ appropriate to the case in figure $11(a)$ with the properties $r\left(z=z_{c}\right)=0, \partial^{2} r / \partial z^{2}\left(z=z_{c}\right)=0$. It then suffices to study the solution of (E 2), (E 3) in the domain $\zeta>0$, with the properties $\hat{\rho}=\hat{\rho}_{0} \zeta+\ldots, \hat{\varphi}^{\prime}=-\frac{1}{3} a_{0} b \zeta+\ldots, \hat{\rho}_{0}=$ const, at small $\zeta$. An affine transformation indicates that we can choose $b=e_{0}=1$, with $a_{1}= \pm 1$ or 0 , leaving the formulation with only two free parameters $a_{0}$ and $\hat{\rho}_{0}$. Note that the flows with $e_{0}<0$ are not considered here. 

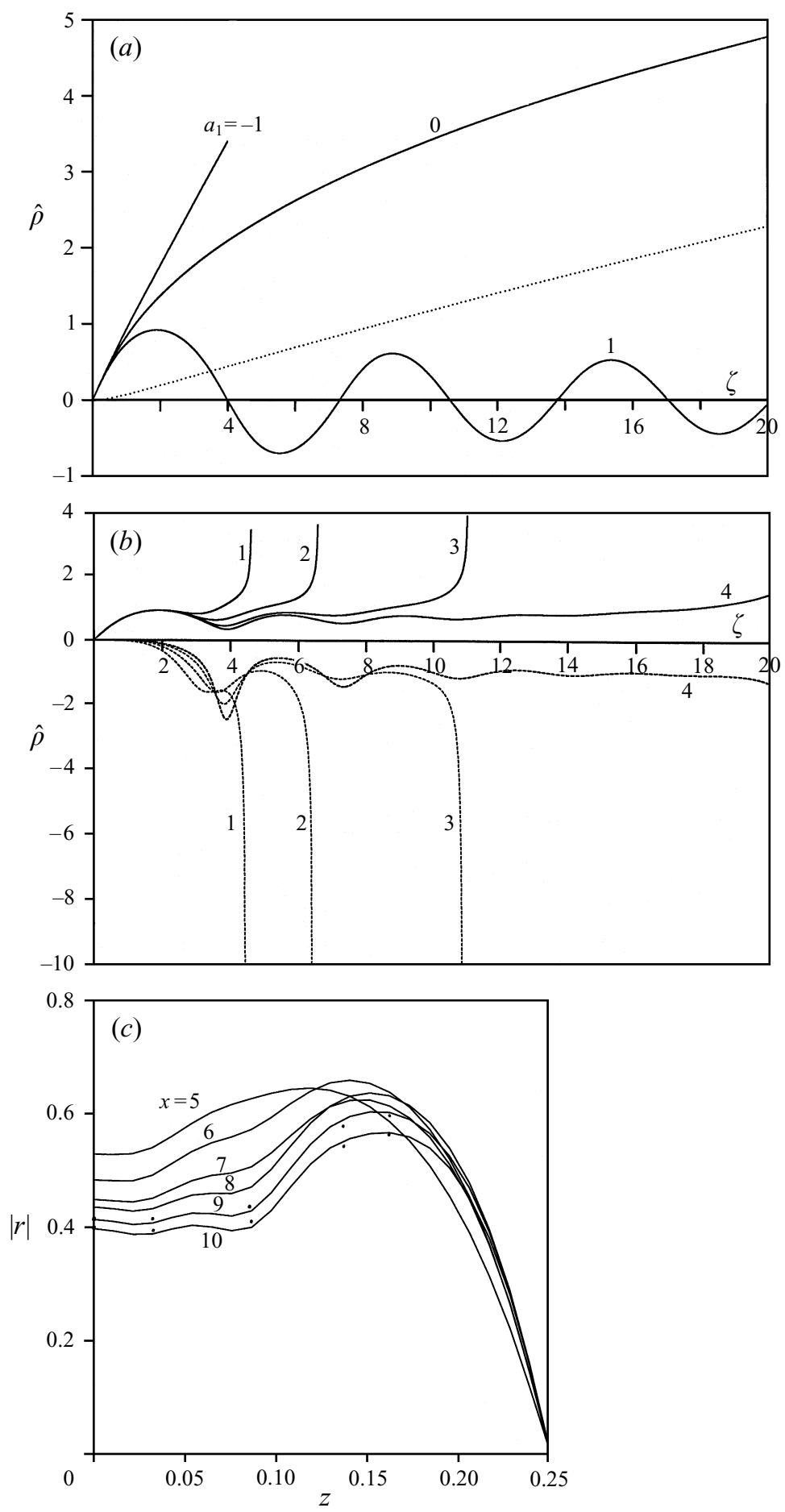

FIGURE 13. (a) Real solutions of (E 2$)$ with $\hat{\varphi}=0, b=e_{0}=1$ and the values of $a_{1}$ marked on the plots (solid); the dashed line illustrates the finite slope in the function $0.1 \hat{\rho}^{2}$ for the case $a_{1}=0$. (b) Solutions of (E 2), (E 3) with $b=e_{0}=a_{1}=1$ and varying $a_{0} ; \hat{\rho}$ (solid) and $\hat{\varphi}$ (dashes) vs. $\zeta$ for the values $a_{0}=0.05,0.03,0.02,0.015$, curves $1-4$ respectively. (c) $|r|$ vs. $z$ from the computation for figure 11(a) at the $x$-stations as marked at the plots (solid), the dots refer to the solution on a finer grid. 
The typical solutions in the case $\hat{\varphi}=0$ due to $a_{0}=0$ are illustrated in figure 13(a). If $a_{1}<0$ then the plot of the amplitude function turns into a straight line at large $\zeta$, with the slope $\mathrm{d} \hat{\rho} / \mathrm{d} \hat{\zeta}=0.8184$ according to computations. Equation (E 2), on the other hand, suggests the behaviour $\hat{\rho}=\zeta\left[-2 a_{1} /\left(\pi e_{0}\right)\right]^{1 / 2}+\ldots$, so that the slope is estimated as 0.7978 , in agreement with the computed value. The solution for $a_{1}>0$ in figure 13(a) tends to become periodic as $\zeta$ increases, in accord with the asymptotic formula $\hat{\rho}=\hat{A} \cos \left(a_{1} \zeta / b+\hat{B}\right)^{1 / 2}+\ldots$ as $\zeta \rightarrow \infty$, with constants $\hat{A}, \hat{B}$. The intermediate regime with $a_{1}=0$ produces a solution with the behaviour $\hat{\rho}=O\left(\zeta^{1 / 2}\right)$, cf. the linear growth of the function $\hat{\rho}^{2}$ shown with the dots in figure 13(a).

In computations for the case $a_{0} \neq 0$ solutions always terminate with a finitedistance singularity of the form $\hat{\rho}=\rho_{s}\left(\zeta_{s}-\zeta\right)^{-1 / 4}+\ldots, \hat{\varphi}^{\prime}=\varphi_{s}\left(\zeta_{s}-\zeta\right)^{-1}+\ldots$, near the singular station, $\zeta \rightarrow \zeta_{s}-0$, with constant coefficients $\rho_{s}, \varphi_{s}$. The location of the singularity is shifted to larger $\zeta$ as $a_{0}$ decreases so that in the limit as $a_{0} \rightarrow 0$ the amplitude $\hat{\rho}$ approaches $|\hat{\rho}|$ of the solution with $a_{1}=1$ in figure $13(a)$. Note that $\hat{\rho}$ remains positive unless the phase is identically zero, cf. figure $13(b)$. The case of small $a_{0}$ seems especially attractive for the asymptotic description of the flow illustrated in figure 11(a), in view of the similarities in the overall structure (decreasing amplitudes and the appearance of shorter cross-scales at larger $x$ ) as well as in the form of the amplitude distribution along the span (non-monotonicity with a larger maximum located closer to the zero point) between the suggested limit and the computational solution of the full equation; see figures $11(a)$ and $13(c)$.

\section{REFERENCES}

Allen, T., Brown, S. N. \& Smith, F. T. 1996 On vortex/wave interactions. Part 2. Originating from axisymmetric flow with swirl. J. Fluid Mech. 325, 145-161.

Bassom, A. P. \& Hall, P. 1990 On the interaction of stationary crossflow vortices and TollmienSchlichting waves in the boundary layer on a rotating disc. Proc. R. Soc. Lond. A 430, 25-55.

Benjamin, T. J. \& FeIR, J. E. 1967 The disintegration of wavetrains on deep water. Part 1. Theory. J. Fluid Mech. 27, 417-430.

Bennett, J., Hall, P. \& Smith, F. T. 1991 The strong nonlinear interaction of Tollmien-Schlichting waves and Taylor-Görtler vortices in curved channel flow. J. Fluid Mech. 223, 475-495.

Benney, D. J. 1961 A nonlinear theory for oscillations in a parallel flow. J. Fluid Mech. 10, 209-236.

BenNey, D. J. \& Lin, C. C. 1960 On the secondary motion induced by oscillations in a shear flow. Phys. Fluids 3, 656-657.

Blackaby, N. D. \& Hall, P. 1995 The nonlinear evolution of the inviscid secondary instability of streamwise vortex structures. Phil. Trans. R. Soc. Lond. A 352, 483-502.

Brown, P. G., Brown, S. N., Smith, F. T. \& Timoshin, S. N. 1993 On the starting process of strongly nonlinear vortex/wave interactions. Mathematika 40, 7-29.

Brown, S. N. \& Smith, F. T. 1996 On vortex/wave interactions. Part 1. Non-symmetrical input and cross-flow in boundary layers. J. Fluid Mech. 307, 101-133.

Cowley, S. J. \& WU, X. 1993 Asymptotic approaches to transition modelling. AGARD Rep. 793.

DAvis, D. A. R. \& Sмiтh, F. T. 1994 Influence of cross-flow on nonlinear Tollmien-Schlichting/vortex interaction. Proc. R. Soc. Lond. A 446, 319-340.

GAJJAR, J. S. B. 1996 On the nonlinear evolution of a stationary cross-flow vortex in a fully three-dimensional boundary-layer flow. J. Fluid Mech. (submitted)

Goldstern, M. E. 1994 Nonlinear interactions between oblique instability waves on nearly parallel shear flows. Phys. Fluids, 6, 724-735.

Goldstein, M. E. \& Chol, S.-W. 1989 Nonlinear evolution of interacting oblique waves on twodimensional shear layers. J. Fluid Mech. 207, 97-120, and Corrigendum 1990, J. Fluid Mech. 216, 659-663. 
Goldstein, M. E. \& LeE, S. S. 1992 Fully-coupled resonant-triad interaction in an adverse-pressuregradient boundary layer. J. Fluid Mech. 245, 523-551.

Goldstein, M. E. \& Leib, S. J. 1989 Nonlinear evolution of oblique waves on compressible shear layers. J. Fluid Mech. 207, 73-96.

Goldstein, M. E. \& Wundrow, D. W. 1995 Interaction of oblique instability waves with weak streamwise vortices. J. Fluid Mech. 284, 377-407.

Hall, P. \& Smith, F. T. 1988 The nonlinear interaction of Tollmien-Schlichting waves and TaylorGörtler vortices in curved channel flows. Proc. R. Soc. Lond. A 417, 255-282.

HaLl, P. \& Smith, F. T. 1989 Nonlinear Tollmien-Schlichting/vortex interaction in boundary layers. Eur. J. Mech. B/Fluids 8, 179-205.

Hall, P. \& Smith, F. T. 1990 Near-planar TS waves and longitudinal vortices in channel flow: nonlinear interaction and focusing. In Instability and Transition (ed. M. Y. Hussaini \& R. G. Voigt), New York.

Hall, P. \& Sмiтh, F. T. 1991 On strongly nonlinear vortex/wave interactions in boundary-layer transition. J. Fluid Mech. 227, 641-666.

Hickernell, F. J. 1984 Time-dependent critical layers in shear flows on the beta-plane. J. Fluid Mech. 142, 431-449.

Hocking, L. M. \& STEWARTSON, K. 1972 On the nonlinear response of a marginally unstable plane parallel flow to a two-dimensional disturbance. Proc. R. Soc. Lond. A 326, 289-313.

Hocking, L. M., Stewartson, K. \& StUART, J. T. 1972 A nonlinear instability burst in plane parallel flow. J. Fluid Mech. 51, 705-735.

Kachanov, YU. S. \& LeVchenko, V. Ya. 1984 The resonant interaction of disturbances at laminarturbulent transition in a boundary layer. J. Fluid Mech. 138, 209-248.

Khokhlov, A. P. 1994 The theory of resonance interaction of Tollmien-Schlichting waves. J. Appl. Mech. Tech. Phys. 34, 508-515.

Klebanoff, P. S., Tidstrom, K. D. \& Sargent, L. M. 1962 The three-dimensional nature of boundary layer instability. J. Fluid Mech. 12, 1-34.

LEIB, S. J. 1991 Nonlinear evolution of subsonic and supersonic disturbances on a compressible free shear layer. J. Fluid Mech. 224, 551-578.

Mankbadi, R. R., Wu, X. \& LeE, S. S. 1993 A critical-layer analysis of the resonant triad in boundary-layer transition: nonlinear interactions. J. Fluid Mech. 256, 85-106.

Maslowe, S. A. 1986 Critical layers in shear flows. Ann. Rev. Fluid Mech. 18, 405-432.

Smith, F. T. \& Bowles, R. I. 1992 Transition theory and experimental comparisons on (I) amplification into streets and (II) a strongly nonlinear break-up criterion. Proc. R. Soc. Lond. A 439, $163-175$.

Smith, F. T., Brown, S. N. \& Brown, P. G. 1993 Initiation of three-dimensional nonlinear transition paths from an inflectional profile. Eur. J. Mech. B/Fluids 12, 447-473.

Smith, F. T. \& Walton, A. G. 1989 Nonlinear interaction of near-planar TS waves and longitudinal vortices in boundary-layer transition. Mathematika 36, 262-289.

Stewart, P. A. \& Smith, F. T. 1992 Three-dimensional nonlinear blow-up from a nearly planar initial disturbance, in boundary-layer transition: theory and experimental comparisons. J. Fluid Mech. 244, 79-100.

Stewartson, K. 1981 Marginally stable inviscid flows with critical layers. IMA J. Appl. Maths 27, $133-175$.

Timoshin, S. N. 1996 Receptivity problems in the weakly-nonlinear stability theory at large Reynolds numbers. In IUTAM Symp. on Nonlinear Instability and Transition in Three-Dimensional Boundary Layers (ed. P. W. Duck \& P. Hall). Kluwer.

Timoshin, S. N. \& Smith, F. T. 1993 On the nonlinear vortex-Rayleigh wave interaction in a boundary-layer flow. Presented at the Intl Workshop on Advances in Analytical Methods in Aerodynamics, 12-14 July 1993, Miedzyzdroje, Poland.

Walton, A. G., Bowles, R. I. \& Smith, F. T. 1994 Vortex-wave interaction in separating flow. Eur. J. Mech. B/Fluids 13, 629-655.

Walton, A. G. \& Smith, F. T. 1992 Properties of strong nonlinear vortex/Tollmien-Schlichting interactions. J. Fluid Mech. 244, 649-676.

Williams, D. R. 1987 Vortical structures in the breakdown stages of transition. In Stability of Time Dependent and Spatially Varying Flows (ed. D. L. Dwoyer \& M. Y. Hussaini), pp. 335-350. Springer. 
Williams, D. R., FASEl, H. \& Hama, F. R. 1984 Experimental determination of the three-dimensional vorticity field in the boundary-layer transition process. J. Fluid Mech. 149, 179-204.

WU, X. 1992 The nonlinear evolution of high-frequency resonant-triad waves in an oscillatory Stokes layer at high Reynolds number. J. Fluid Mech. 245, 553-597.

WU, X. 1993 Nonlinear temporal-spatial modulation of near-planar Rayleigh waves in shear flows: formation of streamwise vortices. J. Fluid Mech. 256, 685-719.

WU, X. 1995 Viscous effects on fully coupled resonant-triad interactions: an analytical approach. J. Fluid Mech. 292, 377-407.

WU, X. \& Cowley, S. J. 1995 On the nonlinear evolution of instability modes in unsteady shear layers: The Stokes layer as a paradigm. Q. J. Mech. Appl. Maths 48, 159-188.

WU, X., LEe, S. S. \& Cowley, S. J. 1993 On weakly nonlinear three-dimensional instability of shear layers to pairs of oblique waves: the Stokes layer as a paradigm. J. Fluid Mech. 253, 681-721.

Wu, X., Stewart, P. A. \& Cowley, S. J. 1996 On the weakly nonlinear development of TollmienSchlichting wavetrains in boundary layers. J. Fluid Mech. 323, 133-171. 